\title{
Properties of Group Five and Group Seven Transactinium Elements
}

\author{
by \\ Philip Arthur Wilk \\ Ph.D. Thesis \\ Department of Chemistry \\ University of California, Berkeley \\ Berkeley, CA 94720 \\ and \\ Nuclear Science Division \\ Ernest Orlando Lawrence Berkeley National Laboratory \\ Berkeley, CA 94720
}

May 2001 


\title{
Properties of Group Five and Group Seven Transactinium Elements
}

\author{
by \\ Philip Arthur Wilk \\ Bachelor of Arts (Reed College) 1995
}

A dissertation submitted in partial satisfaction of the Requirements for the degree of

Doctor of Philosophy

in

Chemistry

in the

GraduAte Division

of the

UNIVERSITY OF CALIFORNIA AT BERKELEY

Committee in charge:

Professor Darleane C. Hoffman, Chair

Professor Heino Nitsche

Professor P. Buford Price

May 2001

This work was supported in part by the Director, Office of Science, Office of High Energy and Nuclear Physics, Division of Nuclear Physics, of the U.S. Department of Energy under Contract No. DE-AC03-76SF00098 
LBNL-47475

\title{
Properties of Group Five and Group Seven Transactinium Elements
}

\author{
Copyright (C) 2001 \\ by \\ Philip Arthur Wilk
}

Portions of this dissertation have been previously published by the author:

P. A. Wilk, K. E. Gregorich, M. B. Hendricks, M. R. Lane, D. M. Lee, C. A. McGrath, D. A. Shaughnessy, D. A. Strellis, E. R. Sylwester, D. C. Hoffman, "Improved Half-life Measurement of ${ }^{224} \mathrm{~Pa}$ and its ${ }^{209} \mathrm{Bi}\left({ }^{18} \mathrm{O}, 3 n\right){ }^{224} \mathrm{~Pa}$ Production Cross Section", Phys. Rev. C 56, 1626-8 (1997) and Erratum, Phys. Rev. C 58, 1352 (1998).

P. A. Wilk, K. E. Gregorich, A. Türler, C. A. Laue, R. Eichler, V. Ninov, J. L. Adams, U. W. Kirbach, M. R. Lane1, D. M. Lee, J. B. Patin, D. A. Shaughnessy, D. A. Strellis, H. Nitsche, and D. C. Hoffman, "Evidence for New Isotopes of Element 107: ${ }^{266} \mathrm{Bh}$ and ${ }^{267} \mathrm{Bh}$ ", Phys. Rev. Lett. 85, 2697 (2000).

R. Eichler, W. Brüchle, Ch. Düllmann, R. Dressler, B. Eichler, H.W. Gäggeler, K.E. Gregorich, T. Häfeli, U. Kirbach, D.C. Hoffman, S. Hübener, D.T. Jost, V.M. Lavanchy, C.A. Laue, H. Nitsche, J. Patin, D. Piguet, M. Schädel, D. Strellis, S. Taut, L. Tobler, Y. Tsyganov, A. Türler, A.Vahle, P.A. Wilk, A.B. Yakushev, P. Zimmermann, Nature 407, 63 (2000).

The U.S. Department of Energy has the right to use this document for any purpose whatsoever including the right to reproduce all or any part thereof. 


\title{
Abstract
}

\section{Properties of Group Five and Group Seven Transactinium Elements}

\author{
by \\ Philip Arthur Wilk \\ Doctor of Philosophy in Chemistry \\ University of California, Berkeley \\ Professor Darleane Hoffman, Chair
}

The detection and positive identification of the short-lived, low cross section isotopes used in the chemical studies of the heaviest elements are usually accomplished by measuring their $\alpha$-decay, thus the nuclear properties of the heaviest elements must be examined simultaneously with their chemical properties. The isotopes ${ }^{224} \mathrm{~Pa}$ and ${ }^{266,267} \mathrm{Bh}$ have been studied extensively as an integral part of the investigation of the heaviest members of the groups five and seven of the periodic table.

The half-life of ${ }^{224} \mathrm{~Pa}$ was determined to be $855 \pm 19 \mathrm{~ms}$ by measuring its $\alpha$-decay using our rotating wheel, solid state detector system at the Lawrence Berkeley National Laboratory 88-Inch Cyclotron. Protactinium was produced by bombardment of a bismuth target. New neutron rich isotopes, ${ }^{267} \mathrm{Bh}$ and ${ }^{266} \mathrm{Bh}$, were produced in bombardments of $\mathrm{a}^{249} \mathrm{Bk}$ target and their decay was observed using the rotating wheel system. The ${ }^{266} \mathrm{Bh}$ that was produced decays with a half-life of $\approx 1 \mathrm{~s}$ by emission of $\alpha$ - 
particles with an average energy of $9.25 \pm 0.03 \mathrm{MeV} .{ }^{267} \mathrm{Bh}$ was observed to decay with a $17_{-6}^{+14}$ s half-life by emission of $\alpha$-particles with an average energy of $8.83 \pm 0.03$ $\mathrm{MeV}$.

The chemical behavior of hahnium, Ha (element 105) was investigated using the fast on-line continuous liquid extraction and detection system SISAK-LISSY. Hahnium was not observed in this experiment following transport and extraction. Protactinium was used as on-line test of the apparatus to determine the experimental efficiency of the entire system. Unfortunately, the amount of protactinium observed after the extraction, compared to the amount produced, was extremely small, only $2.5 \%$. The extraction of the protactinium test isotope indicated the efficiency of the apparatus was too low to observe the extraction of hahnium.

The chemical behavior of oxychloride compounds of bohrium was investigated by isothermal gas adsorption chromatography in a quartz column at 180,150 , and $75^{\circ} \mathrm{C}$. It was found to be less volatile than the corresponding compounds of the lighter group seven homologues, rhenium and technetium, which had been measured previously with the same apparatus. Assuming the bohrium compound to be $\mathrm{BhO}_{3} \mathrm{Cl}$, the evaluated standard adsorption enthalpy, $\Delta \mathrm{H}_{\mathrm{ads}}$, of $\mathrm{BhO}_{3} \mathrm{Cl}$ on the quartz surface was calculated from Monte Carlo fits to the volatility data to be $-75_{-6}^{+9} \mathrm{~kJ} / \mathrm{mol}$. The adsorption enthalpies for $\mathrm{TcO}_{3} \mathrm{Cl}$ and $\mathrm{ReO}_{3} \mathrm{Cl}$ are $-51_{-3}^{+3}$ and $-61_{-3}^{+3} \mathrm{~kJ} / \mathrm{mol}$ respectively. 


\subsection{Dedication}

This thesis is dedicated to the Reed Reactor Facility (RRF) of Reed College,

Portland, Oregon and the Summer School in Nuclear Chemistry that was sponsored by the Division of Nuclear Chemistry and Technology of the American Chemical Society. These two institutions have maintained a commitment to undergraduate education in the field of nuclear chemistry that is unparalleled. It is my hope that Reed will soon replace the long missing radiochemistry professor of the chemistry department to echo this commitment to nuclear and radiochemistry. Without the education and the experience I received from the RRF and the contacts I made at the ACS summer school, I certainly would not be writing this thesis.

Go Griffins! 


\subsection{Acknowledgments}

First and foremost, I would like to thank Professor Darleane C. Hoffman, for giving me the chance to pursue nuclear chemistry at Berkeley. I am most indebted to the support and advice of the entire Hoffman group and affiliates overseas that I have had a chance to work with, especially the veteran group members Dawn Shaughnessy, Joshua Patin, Diana Lee, and Ken Gregorich for their extensive help. Its been great to work with you all during such exciting times in nuclear chemistry!

I would like to give a special thank-you to Charlise Tiee and Scott Locklin for being great friends and colleagues. I quite literally could not have done it without you two. I would also like to thank my parents for their support throughout my entire education.

I would like to thank the staff and crew of the LBNL 88-Inch Cyclotron for their assistance and support. I am indebted to the Office of Science, Office of Basic Energy Research, Division of Chemical Sciences, of the U.S. Department of Energy, for making the ${ }^{249} \mathrm{Bk}$ target material available through the transplutonium element production program at the Oak Ridge National Laboratory. This work was supported in part by the Director, Office of Science, Office of High Energy and Nuclear Physics, Division of Nuclear Physics, of the U. S. Department of Energy under Contract No. DE-AC03-76SF00098. 


\subsection{Table of Contents}

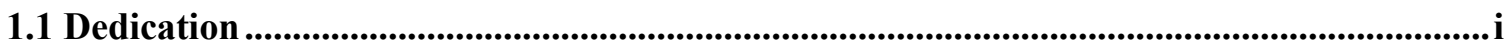

1.2 Acknowledgments .........................................................................................................................ii

1.3 Table of Contents .........................................................................................................................iii

1.4 List of Figures...................................................................................................................................v

1.5 List of Tables .............................................................................................................................................vi

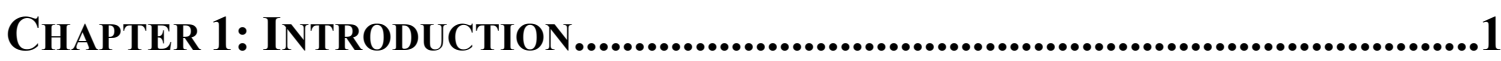

1.1 History .......................................................................................................................................... 1

1.2 Scope of Thesis ...............................................................................................................................

CHAPTER 2: THEORY .......................................................................................

2.1 Nuclear Theory

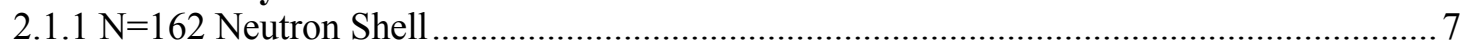

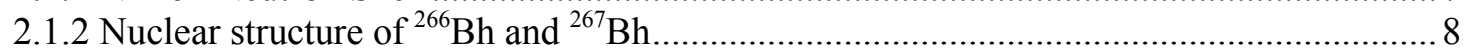

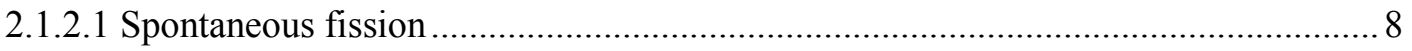

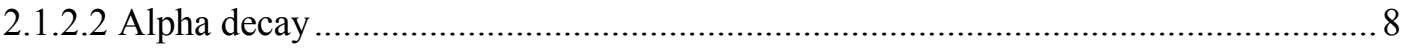

2.1.2.3 Electron-capture decay ..............................................................................

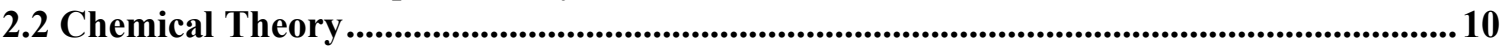

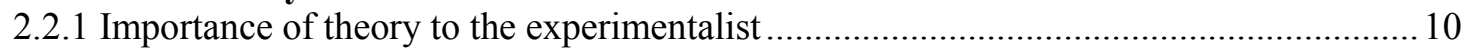

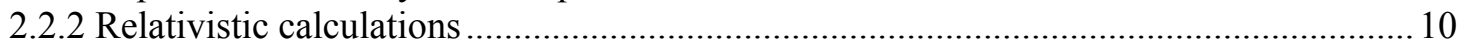

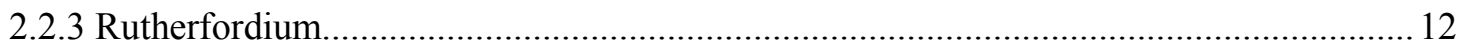

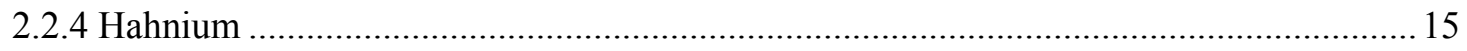

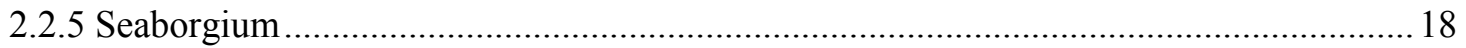

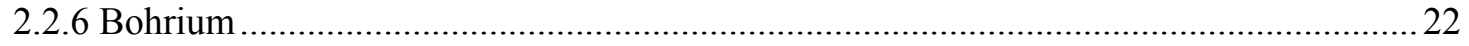

ChAPTER 3: INSTRUMENTATION AND TECHNIQUES ..................................... 24

3.1 Accelerator .......................................................................................................................... 24

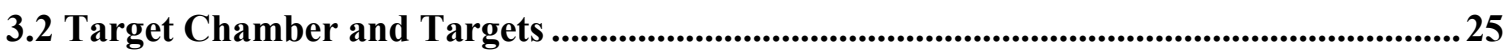

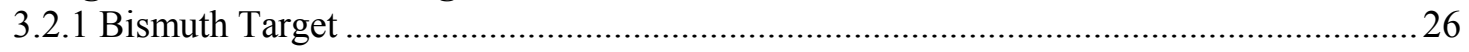

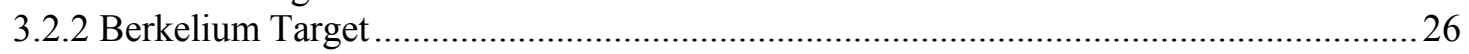

3.3 Production Reactions............................................................................................28

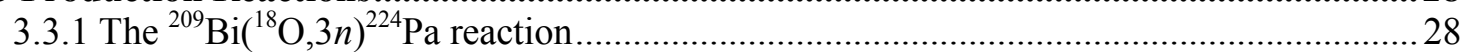

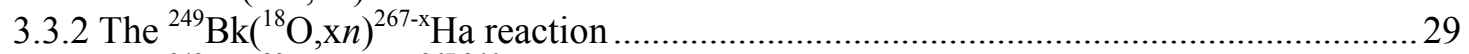

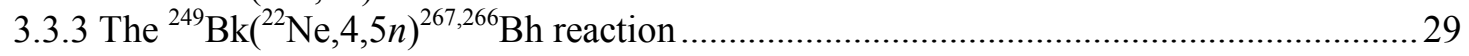

3.4 Collection and Transport ............................................................................................................ 29

3.5 Detection ...................................................................................................................................... 30

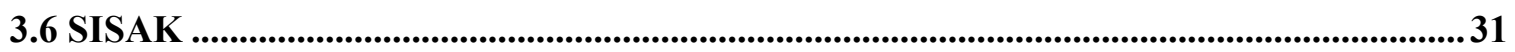

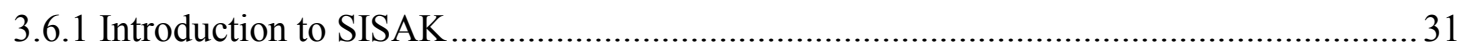

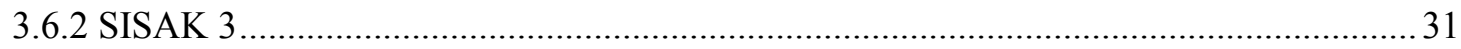

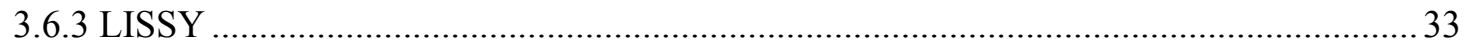

3.7 Data Acquisition ............................................................................................................................... 34 
Chapter 4: Group Five Elements ..................................................... 37

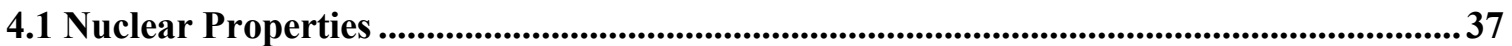

4.1.1 Cross section for the ${ }^{209} \mathrm{Bi}\left({ }^{18} \mathrm{O}, 3 n\right){ }^{224} \mathrm{~Pa}$ reaction and half-life of ${ }^{224} \mathrm{~Pa}$............................. 37

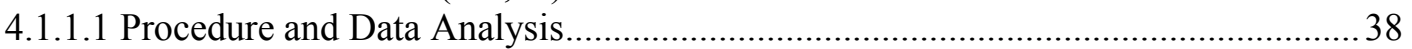

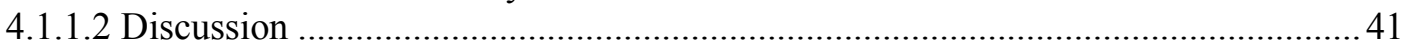

4.2 Chemical Properties............................................................................................................................... 41

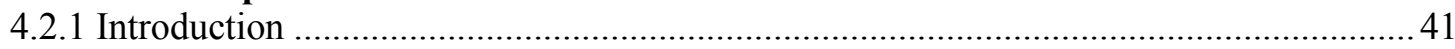

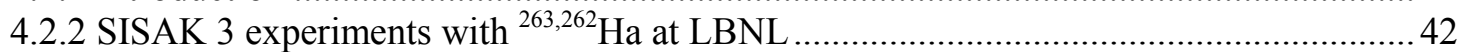

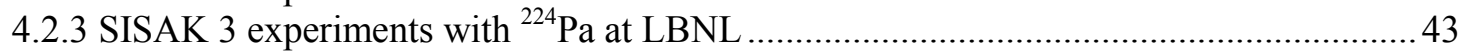

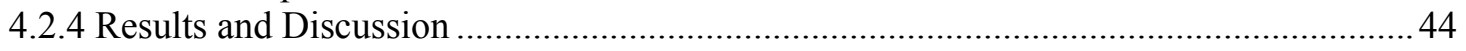

CHAPTER 5: Group SEVEN ELEMENTS .................................................. 49

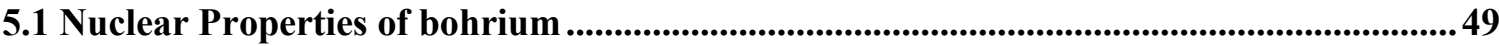

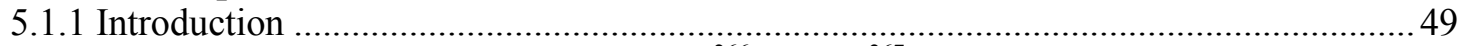

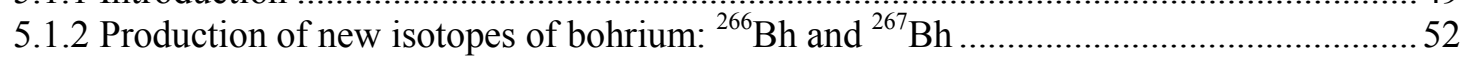

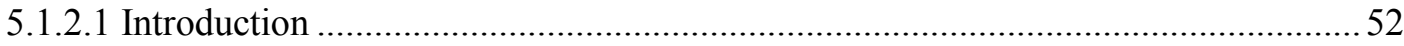

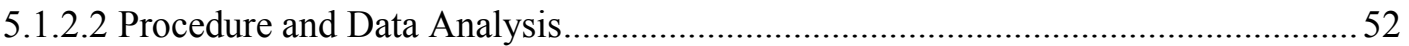

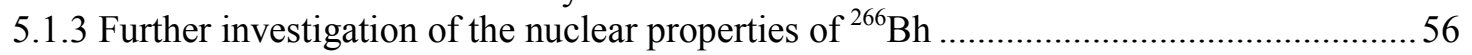

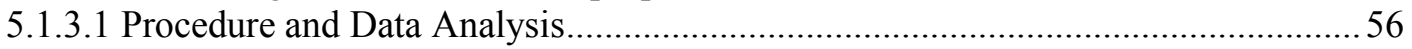

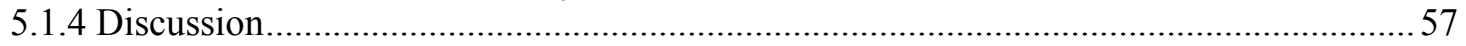

5.2 Chemical properties of bohrium................................................................................................59

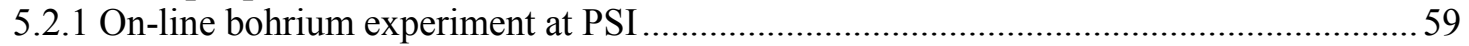

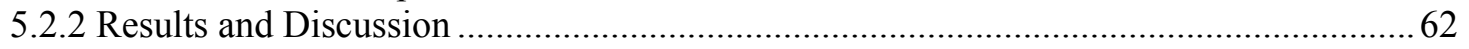

Chapter 6: Conclusions and Future Work .................................. 63

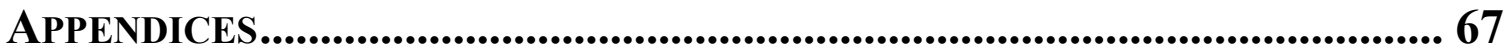

Appendix A: Glossary of Acronyms................................................................................................67

Appendix B: List of primary and contributed publications and talks......................................68

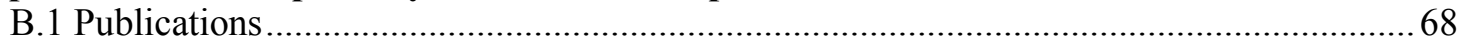

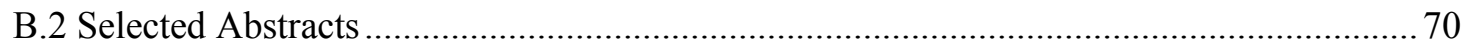

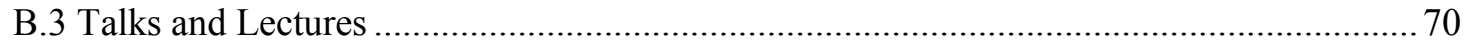

Appendix C: Review Articles and General Texts on Nuclear Chemistry .................................. 71

Appendix D: Photographs and Color Pictures..................................................................................... 72

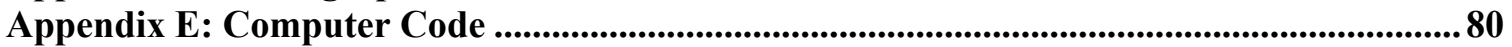

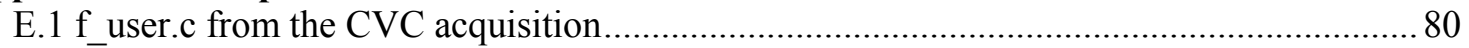

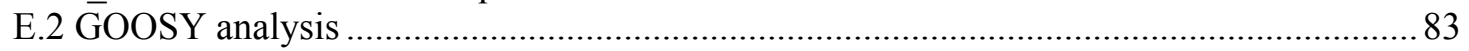

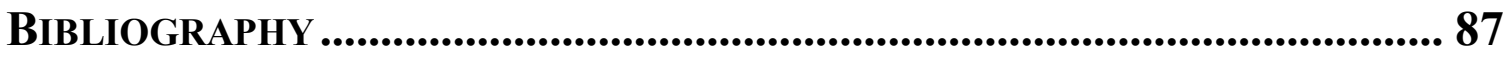




\subsection{List of Figures}

Figure 1.1 The Periodic Table as arranged by Glenn T. Seaborg, 1945 [Seaborg 1945]. Picture courtesy of the LBNL archives. ................................................................................................................

Figure 1.2 Current Periodic Table...................................................................................................................4

Figure 2.1 Photolysis of chromium hexacarbonyl followed by the formation of the solvated chromium pentacarbonyl species. ....................................................................................................................................21

Figure 3.1 Collection and Transport System ....................................................................................................25

Figure 3.2 Parent-Daughter Mode Schematic........................................................................................................30

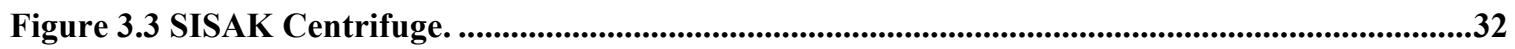

Figure 3.4 The $\alpha$-LS “Meander" Cell ..................................................................................................................34

Figure 4.1 The $\alpha$-particle spectrum of products of the ${ }^{209} \mathrm{Bi}$ and ${ }^{18} \mathrm{O}$ reaction collected over a 1.3-hour

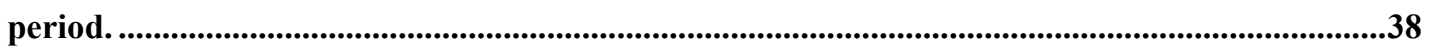

Figure 4.2 Contour plot of $\alpha$-events occurring within $2 \mu$ in opposite detectors ......................................39

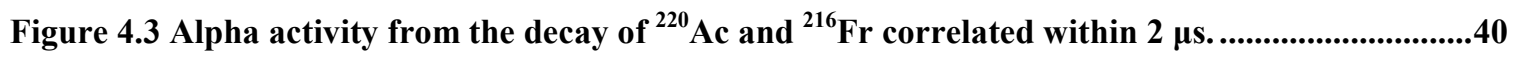

Figure 4.4 Protactinium generator ..................................................................................................................45

Figure 4.5 An example of a SISAK configuration [Omtvedt 2001].............................................................47

Figure 5.1 Partial decay chain of ${ }^{266} \mathrm{Bh}$ and ${ }^{267} \mathrm{Bh}$. Decay properties of ${ }^{266} \mathrm{Bh}$ and ${ }^{267} \mathrm{Bh}$ in the dashed boxes are as measured during these experiments. ......................................................................................52

Figure 5.2 The $\alpha$-particle spectrum measured over the entire 62-hour experiment in detector pair

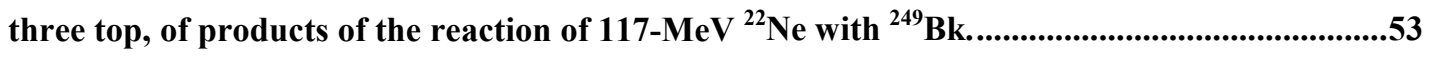

Figure 5.3 OLGA III [Eichler 2001] ...............................................................................................................61 


\subsection{List of Tables}

Table 1.1 Transmendelevium nomenclature used in this work.............................................................5

Table 2.1 Electronic ground state structure of the group five elements ..................................................15

Table 2.2 Electronic ground state structure of the group six elements.................................................18

Table 5.1 List of correlations between parent events $\left(8.6<\mathrm{E}_{\alpha}<10.5 \mathrm{MeV}\right)$ and daughter events

$\left(8.2<\mathbf{E}_{\alpha}<8.7 \mathrm{MeV}\right)$. The initiating parent event, each subsequent $\alpha$-decay that occurred within the energy window, its isotopic assignment, alpha energy, and time are listed for each event. ..55

Table 5.2 Experimental cross sections and SPIT predictions for the reactions ${ }^{22} \mathrm{Ne}$ on ${ }^{249} \mathrm{Bk}$ and ${ }^{22} \mathrm{Ne}$

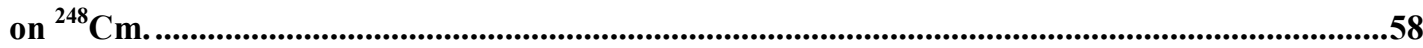

Table 5.3 Reactions analogous to the ${ }^{22} \mathrm{Ne}$ on ${ }^{249} \mathrm{Bk}$ reaction showing similar $4 n$ and $5 n$ exit channel

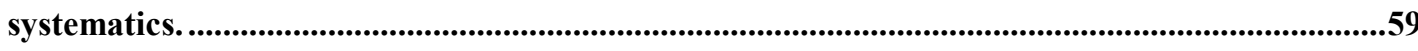




\section{Chapter 1: Introduction}

\subsection{History}

Nuclear and chemical techniques can be complementary methods of investigating the properties of the transactinium elements. Radiochemical techniques have been and continue to be a powerful tool for study of the physical properties of the heavy elements. The unique $\mathrm{Z}$ resolution of chemical techniques can be utilized in situations where purely physical techniques do not work well. The discoveries of new elements through mendelevium (element 101) and their properties were at first purely a chemical endeavor, but nuclear techniques played a larger and larger role as time went on. Mendelevium was the first element to be discovered via atom-at-a-time chemical discovery and the last element discovered by chemical techniques.

The current work on expanding the periodic table has its foundation in the research of Dmitri Mendeleev ${ }^{1}$, the father of the modern periodic table. He proposed in 1869 a table of elements ordered by increasing atomic weight. This however, was not his greatest contribution to science as he was not the first to suggest a systematic organization of the elements based on atomic weight. In fact, nor was the system that he did propose, unique, as Lothar Meyer independently proposed almost an identical table at about the same time. Indeed, both scientists also left gaps for yet to be discovered elements, but Mendeleev's great insight took his invention one step further: He used his periodic table to identify errors in the published measurements of the

\footnotetext{
${ }^{1}$ also spelled Mendelejeff
} 
known elements, and quite extraordinarily, used the table to predict the properties of six undiscovered elements. This is not unlike the work that is the basis for some of this thesis, in which the chemical properties of the heaviest elements are predicted.

Mendeleev's table was a great success and many new experiments quickly validated its predictions. There were two notable changes to this table before its ordering system had to be changed to an organization based on atomic number. The first was the addition of a new group of elements due to the discovery of the noble gasses by Lord Rayleigh and William Ramsay. The second was the addition of the rare earth elements - the lanthanide series. These successful changes further underscored the validity of the Mendeleev Table. Unfortunately, there was an unavoidable problem of a few elements, that when arranged in order of increasing atomic weight, did not have their positions match their corresponding chemical properties. This disparity occurred for example, with argon and potassium, tellurium and iodine, as well as cobalt and nickel where their atomic weights were reversed. The solution to this problem was to eventually abandon ordering the table by atomic weight, and utilize a different, but related property: the atomic number.

The next permutation of the periodic table was proposed by H.G.J. Mosely (1913) who arranged the elements according to their X-ray spectra instead of atomic weight. He showed that the atomic number is proportional to the square root of the $\mathrm{x}$ ray energy, and proportional to the number of electrons orbiting the nucleus that dictates chemical properties. Arranging the table according to atomic number led to a table that was nearly identical to Mendeleev Table, but avoided problems with the chemical behavior of the elements whose atomic weights were reversed. This method 
of ordering the table is essentially the one we use today.

No changes were made to the structure of the Periodic Table until 1944, when Glenn T. Seaborg postulated the existence of the actinide series, analogous to the lanthanide series (see Figure 1.1) [Seaborg 1945]. This seemingly simple extrapolation was not obvious given the data at the time, and led to the nearly immediate discovery of americium and curium. Before Seaborg's formulation of the actinide hypothesis, it was anticipated that these two elements would be part of a uranium-like group, and could be expected to behave like neptunium and plutonium. Scientists were, before Professor Seaborg's great insight, anticipating the wrong oxidation state for these elements.

Figure 1.1 The Periodic Table as arranged by Glenn T. Seaborg, 1945 [Seaborg 1945]. Picture courtesy of the LBNL archives.

PERIODIC TABLE SHOWING HEAVY ELEMENTS AS MEMBERS

OF AN ACTINIDE SERIES

Arrangement by Glenn T. Seaborg, 1945

\begin{tabular}{|c|c|c|c|c|c|c|c|c|c|c|c|c|c|c|c|c|c|}
\hline $\begin{array}{c}1 \\
H \\
H \\
\end{array}$ & & & & & & & & & & & & & & & & $\underset{1}{\mathrm{H}}$ & $\begin{array}{c}2 \\
\mathrm{He}_{\mathrm{e}} \\
4.003\end{array}$ \\
\hline $\begin{array}{c}3 \\
\mathrm{Li} \\
6.940 \\
\end{array}$ & $\begin{array}{c}4 \\
\mathrm{Be} \\
902\end{array}$ & & & & & & & & & & & \begin{tabular}{|c|}
5 \\
8 \\
10.92 \\
\end{tabular} & \begin{tabular}{|c|}
6 \\
6 \\
12.010 \\
\end{tabular} & \begin{tabular}{|c|} 
\\
$N$ \\
14.000 \\
\end{tabular} & \begin{tabular}{|c|c}
8 \\
0 \\
06.000 \\
\end{tabular} & \begin{tabular}{|c|}
$\mathbf{9}$ \\
$\mathbf{F}$ \\
1900 \\
\end{tabular} & $\begin{array}{c}10 \\
\mathrm{Ne}^{2} \\
20.193\end{array}$ \\
\hline \begin{tabular}{|c|}
11 \\
$\mathrm{No}$ \\
22.997 \\
\end{tabular} & \begin{tabular}{|c|c|}
12 \\
$M_{9}$ \\
24.32 \\
\end{tabular} & $\begin{array}{c}13 \\
A 1 \\
2697 \\
269\end{array}$ & & & & & & & & & & \begin{tabular}{|c|}
13 \\
$A 1$ \\
26.97 \\
\end{tabular} & \begin{tabular}{|c|}
14 \\
51 \\
2806 \\
\end{tabular} & \begin{tabular}{|c|}
15 \\
$p$ \\
30.98 \\
\end{tabular} & \begin{tabular}{|l}
16 \\
5 \\
32.06 \\
\end{tabular} & $\begin{array}{c}17 \\
c 1 \\
35.457 \\
\end{array}$ & \begin{tabular}{|c|}
18 \\
A \\
39.949 \\
\end{tabular} \\
\hline \begin{tabular}{c|c|}
19 \\
$k$ \\
30006
\end{tabular} & $\begin{array}{l}20 \\
\mathrm{ca} \\
\text { to. }\end{array}$ & $\begin{array}{c}21 \\
\mathrm{Sc} \\
45 . \% 0\end{array}$ & $\begin{array}{c}22 \\
T i \\
T i \\
4790\end{array}$ & $\begin{array}{c}23 \\
v \\
\text { so }\end{array}$ & $\begin{array}{l}24 \\
C_{r} \\
02 O A\end{array}$ & \begin{tabular}{|l}
25 \\
$\mathrm{Mn}$ \\
3493
\end{tabular} & $\begin{array}{c}26 \\
\mathrm{Fo} \\
\text { 55.85 }\end{array}$ & $\begin{array}{c}27 \\
60 \\
5098 \\
509\end{array}$ & $\begin{array}{c}28 \\
\mathrm{Ni} \\
\text { se69 }\end{array}$ & \begin{tabular}{|c|}
29 \\
$6 u$ \\
63.57 \\
\end{tabular} & \begin{tabular}{|c|}
30 \\
$2 n$ \\
6538 \\
\end{tabular} & $\begin{array}{l}31 \\
60 \\
69 \pi 2 \\
\end{array}$ & $\begin{array}{l}32 \\
60 \\
7260\end{array}$ & \begin{tabular}{|c|}
33 \\
As \\
74.91 \\
\end{tabular} & \begin{tabular}{|c|}
34 \\
50 \\
T6.96 \\
\end{tabular} & \begin{tabular}{|l}
35 \\
Br \\
rasie \\
\end{tabular} & $\begin{array}{l}36 \\
K_{1} \\
\text { ost } \\
\end{array}$ \\
\hline $\begin{array}{c}37 \\
\text { Rb } \\
\text { as.as }\end{array}$ & \begin{tabular}{|l|}
38 \\
$S r$ \\
Sit63
\end{tabular} & $\begin{array}{c}39 \\
y \\
\text { on } x\end{array}$ & $\begin{array}{l}40 \\
\mathrm{Zr} \\
2128\end{array}$ & $\begin{array}{c}4 ! \\
c b \\
=221\end{array}$ & $\begin{array}{l}42 \\
\text { Mo } \\
25 \text { is }\end{array}$ & 43 & \begin{tabular}{|c|}
44 \\
$R u$ \\
Ru \\
101.7
\end{tabular} & $\begin{array}{c}45 \\
\text { Rh } \\
102.91\end{array}$ & $\begin{array}{c}46 \\
\mathrm{Pd} \\
1067\end{array}$ & $\begin{array}{c}47 \\
A_{g} \\
\text { 10 } 8 \text { or }\end{array}$ & \begin{tabular}{|c|}
48 \\
Cd \\
112.4.
\end{tabular} & $\begin{array}{c}49 \\
\text { in } \\
11476\end{array}$ & \begin{tabular}{|c|}
50 \\
Sn \\
11070 \\
\end{tabular} & $\begin{array}{c}51 \\
56 \\
12176\end{array}$ & $\begin{array}{c}52 \\
\text { Te } \\
\text { ant }\end{array}$ & $\begin{array}{c}53 \\
1 \\
12692 \\
\end{array}$ & \begin{tabular}{|r|}
54 \\
$x e$ \\
131.3 \\
\end{tabular} \\
\hline $\begin{array}{r}55 \\
65 \\
65 \\
13291 \\
\end{array}$ & \begin{tabular}{|c|}
56 \\
80 \\
13736 \\
\end{tabular} & 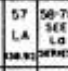 & $\begin{array}{r}72 \\
11 \\
1736.6\end{array}$ & \begin{tabular}{c|c}
73 \\
70 \\
100 \\
\end{tabular} & $\begin{array}{c}4 \\
\text { W } \\
\text { 1639? }\end{array}$ & \begin{tabular}{|c|}
75 \\
$R e$ \\
Re \\
10631 \\
\end{tabular} & $\begin{array}{c}76 \\
0 \text { s } \\
1902 \\
\end{array}$ & \begin{tabular}{|c|}
77 \\
18 \\
153.1 \\
193,
\end{tabular} & \begin{tabular}{|c|}
78 \\
Pf \\
195.23 \\
\end{tabular} & $\begin{array}{c}79 \\
\mathrm{Au} \\
1972 \\
\end{array}$ & $\begin{array}{l}80 \\
\mathrm{Hg} \\
20061\end{array}$ & $\begin{array}{c}81 \\
T 1 \\
20439\end{array}$ & $\mid \begin{array}{c}\mathrm{B2} \\
\mathrm{Pb} \\
207.21\end{array}$ & $\begin{array}{c}83 \\
81 \\
8000 \\
20000\end{array}$ & $\begin{array}{l}84 \\
\text { Po }\end{array}$ & 85 & \begin{tabular}{|l|}
86 \\
$R n$ \\
222 \\
222
\end{tabular} \\
\hline 87 & $\begin{array}{l}\mathbf{8 B} \\
\mathrm{Ro}\end{array}$ & 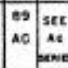 & $\begin{array}{l}90 \\
T h\end{array}$ & $\begin{array}{l}91 \\
\text { Po }\end{array}$ & \begin{tabular}{l|l}
92 & 93 \\
\end{tabular} & Tyu & 95 & 96 & & & & & & & & & \\
\hline
\end{tabular}

\begin{tabular}{|c|c|c|c|c|c|c|c|c|c|c|c|c|c|c|c|}
\hline $\begin{array}{l}\text { LANTHANIDE } \\
\text { SERIES }\end{array}$ & $\begin{array}{l}57 \\
50 \\
1098 \\
109\end{array}$ & $\begin{array}{r}58 \\
6 e \\
6003 \\
100\end{array}$ & \begin{tabular}{|c|}
59 \\
$\mathrm{Pr}$ \\
140.022
\end{tabular} & \begin{tabular}{|l|}
60 \\
Nd \\
i4427
\end{tabular} & 61 & $\begin{array}{c}62 \\
5 m \\
15043 \\
\end{array}$ & $\begin{array}{c}63 \\
E_{0} \\
520 \\
520\end{array}$ & $\begin{array}{r}64 \\
\text { Gd } \\
\text { Gs6.9 } \\
\end{array}$ & $\begin{array}{l}65 \\
\mathrm{~Tb} \\
1992 \\
\end{array}$ & 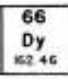 & $\begin{array}{l}67 \\
\text { Ho } \\
16539 \\
\end{array}$ & $\begin{array}{l}68 \\
\text { Er } \\
1672 \\
\end{array}$ & $\begin{array}{c}69 \\
\mathrm{Tm} \\
1694 \\
\end{array}$ & $\begin{array}{l}70 \\
\text { Yb } \\
133.04\end{array}$ & \begin{tabular}{|l|}
71 \\
Lu \\
174.99
\end{tabular} \\
\hline $\begin{array}{l}\text { ACTINIDE } \\
\text { SERIES }\end{array}$ & $\begin{array}{l}89 \\
A C\end{array}$ & $\begin{array}{c}90 \\
\text { Th } \\
232.12\end{array}$ & $\begin{array}{l}91 \\
\mathrm{PO} \\
231\end{array}$ & $\begin{array}{c}92 \\
\text { u } \\
238 \text { or }\end{array}$ & $\begin{array}{l}93 \\
\text { Np } \\
237\end{array}$ & $\begin{array}{l}94 \\
\text { Pu }\end{array}$ & 95 & \begin{tabular}{|l|}
96 \\
\end{tabular} & & & & & & & \\
\hline
\end{tabular}


Figure 1.2 Current Periodic Table

\begin{tabular}{|c|c|c|c|c|c|c|c|c|c|c|c|c|c|c|c|c|c|}
\hline $\begin{array}{l}\mathbf{1} \\
\mathbf{H}\end{array}$ & & & & & & & & & & & & & & & & & \begin{tabular}{|l|}
2 \\
$\mathrm{He}$
\end{tabular} \\
\hline 3 & 4 & & & & & & & & & & & 5 & 6 & 7 & 8 & 9 & 10 \\
\hline $\mathbf{L i}$ & $\mathrm{Be}$ & & & & & & & & & & & B & C & $\mathbf{N}$ & O & $\mathbf{F}$ & $\mathrm{Ne}$ \\
\hline$\overline{11}$ & 12 & & & & & & & & & & & 13 & 14 & $\overline{15}$ & 16 & 17 & 18 \\
\hline $\mathrm{Na}$ & Mg & 3 & 4 & 5 & 6 & 7 & 8 & 9 & 10 & 11 & 12 & $\mathrm{Al}$ & $\mathbf{S i}$ & $\mathbf{P}$ & $\mathbf{S}$ & $\mathrm{Cl}$ & Ar \\
\hline$\overline{19}$ & 20 & 21 & 22 & 23 & 24 & 25 & 26 & \begin{tabular}{|l|}
27 \\
\end{tabular} & 28 & 29 & 30 & 31 & 32 & 33 & 34 & 35 & 36 \\
\hline $\mathbf{K}$ & $\mathbf{C a}$ & Sc & $\mathbf{T i}$ & $\mathbf{V}$ & $\mathrm{Cr}$ & Mn & $\mathbf{F e}$ & Co & $\mathbf{N i}$ & $\mathbf{C u}$ & $\mathrm{Zn}$ & $\mathbf{G a}$ & Ge & As & $\mathrm{Se}$ & $\mathrm{Br}$ & $\mathbf{K r}$ \\
\hline 37 & 38 & 39 & 40 & 41 & 42 & 43 & 44 & 45 & 46 & 47 & 48 & 49 & 50 & 51 & 52 & 53 & 54 \\
\hline $\mathbf{R b}$ & $\mathrm{Sr}$ & $\mathbf{Y}$ & $\mathbf{Z r}$ & $\mathrm{Nb}$ & Mo & Tc & $\mathbf{R u}$ & $\mathbf{R h}$ & $\mathbf{P d}$ & Ag & Cd & In & Sn & $\mathrm{Sb}$ & $\mathbf{T e}$ & I & $\mathrm{Xe}$ \\
\hline 55 & 56 & 57 & 72 & 73 & \begin{tabular}{|l|}
74 \\
\end{tabular} & 75 & 76 & 77 & 78 & 79 & 80 & 81 & 82 & 83 & 84 & $\overline{85}$ & 86 \\
\hline Cs & $\mathbf{B a}$ & La & Hf & $\mathbf{T a}$ & W & $\boldsymbol{R e}$ & Os & Ir & $\mathbf{P t}$ & $\mathrm{Au}$ & $\mathrm{Hg}$ & $\mathrm{Tl}$ & $\mathbf{P b}$ & $\mathbf{B i}$ & Po & At & $\mathbf{R n}$ \\
\hline 87 & 88 & 89 & $\overline{104}$ & 105 & $\begin{array}{l}106 \\
\end{array}$ & \begin{tabular}{|l|}
107 \\
\end{tabular} & 108 & 109 & 110 & 111 & 112 & & 114 & & 116 & & 118 \\
\hline Fr & $\mathbf{R a}$ & Ac & $\mathbf{R f}$ & $\mathbf{H a}$ & Sg & Bh & Hs & Mt & & & & & & & & & \\
\hline
\end{tabular}

\begin{tabular}{|c|c|c|c|c|c|c|c|c|c|c|c|c|c|c|}
\hline Lanthanides & $\begin{array}{l}58 \\
\mathrm{Ce}\end{array}$ & $\begin{array}{l}59 \\
\text { Pr }\end{array}$ & $\begin{array}{l}60 \\
\mathrm{Nd}\end{array}$ & $\begin{array}{l}61 \\
\text { Pm }\end{array}$ & $\begin{array}{l}62 \\
\mathrm{Sm}\end{array}$ & $\begin{array}{l}63 \\
\mathrm{Eu}\end{array}$ & $\begin{array}{l}64 \\
\text { Gd }\end{array}$ & $\begin{array}{l}65 \\
\text { Tb }\end{array}$ & $\begin{array}{l}66 \\
\text { Dy }\end{array}$ & $\begin{array}{l}67 \\
\text { Ho }\end{array}$ & $\begin{array}{c}68 \\
\text { Er }\end{array}$ & \begin{tabular}{|l|}
69 \\
Tm
\end{tabular} & $\begin{array}{l}70 \\
Y b\end{array}$ & $\begin{array}{l}71 \\
\mathrm{Lu}\end{array}$ \\
\hline Actinides & $\begin{array}{l}90 \\
\text { Th }\end{array}$ & $\begin{array}{l}91 \\
\mathrm{~Pa}\end{array}$ & $\begin{array}{r}92 \\
\mathrm{U}\end{array}$ & $\begin{array}{l}93 \\
\text { Np }\end{array}$ & $\begin{array}{l}\overline{94} \\
\mathrm{Pu}\end{array}$ & $\begin{array}{l}95 \\
\text { Am }\end{array}$ & $\begin{array}{l}96 \\
\mathrm{Cm}\end{array}$ & $\begin{array}{l}97 \\
\mathrm{Bk}\end{array}$ & $\begin{array}{l}98 \\
\text { Cf }\end{array}$ & $\begin{array}{l}99 \\
\text { Es }\end{array}$ & $\begin{array}{l}100 \\
\text { Fm }\end{array}$ & $\begin{array}{l}101 \\
\text { Md }\end{array}$ & $\begin{array}{l}102 \\
\text { No }\end{array}$ & $\begin{array}{c}103 \\
\mathrm{Lr}\end{array}$ \\
\hline
\end{tabular}

Periodic trends held true through the end of the actinide series and the elements as they were discovered behaved mostly as expected. The chemistry of the transcurium actinides resemble the lanthanides to a remarkable extent, filling the $5 f$ shell versus the $4 f$. Except for the deviation of nobelium, which is more stable in aqueous solution in the $2+$ oxidation state due to the extra stability imparted by the half-filled $5 f$-shell, the trivalent ions of all the other transcurium actinides are most stable in aqueous solution. As expected, the series exhibits an ionic radius contraction analogous to the lanthanide contraction due to the ineffective shielding of nuclear charge by the $f$-orbital electrons. In fact, trivalent actinides resemble the lanthanides to such an extent that ordinarily a lanthanide is used as a carrier during precipitations [Higgins 1960]. 
With the end of the actinide series comes the expected beginning of a new transition metal series characterized by the filling of the $6 d$-orbitals. The names of the elements after mendelevium are listed in Table 1.1. For consistency with previous work, as well as to honor Otto Hahn and respect the wishes of Glenn Seaborg, I will continue to use the name "hahnium" for element 105. The expected transition metal behavior, however, is now likely to be even more influenced by the so-called "relativistic effect", which increases as a function of $Z^{2}$. This effect on these elements will be discussed further in following sections.

Table 1.1 Transmendelevium nomenclature used in this work.

\begin{tabular}{cll} 
Atomic number & \multicolumn{1}{c}{ Element name } & IUPAC name [IUPAC 1997] \\
\hline 102 & nobelium, No & nobelium, No \\
103 & lawrencium, $\mathrm{Lr}$ & lawrencium, $\mathrm{Lr}$ \\
104 & rutherfordium, Rf & rutherfordium, Rf \\
105 & hahnium, Ha & dubnium, $\mathrm{Db}$ \\
106 & seaborgium, $\mathrm{Sg}$ & seaborgium, $\mathrm{Sg}$ \\
107 & bohrium, Bh & bohrium, $\mathrm{Bh}$ \\
108 & hassium, Hs & hassium, $\mathrm{Hs}$ \\
109 & meitnerium, $\mathrm{Mt}$ & meitnerium, Mt
\end{tabular}

\subsection{Scope of Thesis}

A brief overview of both the pertinent nuclear theory and chemical theory will be given for background to the research contained within this thesis. The measurement of the nuclear properties of a few of the isotopes that were used for chemical studies accounts for the bulk of the research that is the basis of this dissertation. The measured nuclear properties of these isotopes are of considerable interest by themselves, and will be discussed at some length. The chemical properties and periodicity of the periodic table will be discussed as they pertain to the transactinium members of the 
fifth and the seventh groups. The majority of this work is a collaborative effort between many scientists and several countries with different researchers taking major responsibilities for various aspects of the research at various times. I participated in investigations of the chemistry of the heaviest members of the fifth and seventh group of the periodic table and a discussion of these experiments is included. As mentioned, a major portion of my responsibility lay with determining the nuclear properties of some of the isotopes necessary for the subsequent chemistry experiments in which I participated. 


\section{Chapter 2: Theory}

\subsection{Nuclear Theory}

\subsection{1 $\mathrm{N}=162$ Neutron Shell}

Recent calculations by nuclear theorists indicate the presence of deformed nuclear shells at $\mathrm{N}=162$ and $\mathrm{Z}=108$ (neutron number and proton number). These shells should significantly stabilize the nucleus against spontaneous fission (SF) [Smolańczuk 1995, 1997, Swiatecki 1996, Möller 1998]. Recent experimental results [Lougheed 1997a] showed that isotopes in this region have relatively long half-lives and decay primarily by $\alpha$-particle emission rather than spontaneous fission, confirming the deformed shell predictions. These results have generated much interest for two main reasons. First, it now seems possible to identify many isotopes between the presently known heavy elements and the spherical superheavy elements predicted earlier to exist near the closed spherical proton and neutron shells at $Z=114$ and $\mathrm{N}=184$ [Armbruster 1997, Oganessian 1997]. Secondly, half-lives for isotopes of element 106-109 will be long enough to make the determination of the chemical properties of these elements possible. In addition, the success of the theoretical modeling of heavy element masses and stability has increased confidence in calculations of the stability of spherical superheavy elements. Measurements of the $\alpha-$ decay energies and half-lives of these isotopes near the $\mathrm{N}=162$ deformed shell are needed to determine the masses of these isotopes and refine the calculations of the strength and extent of the shell effects, and could give information on the magnitude 
of the nuclear deformations.

\subsubsection{Nuclear structure of ${ }^{266} \mathrm{Bh}$ and ${ }^{267} \mathrm{Bh}$}

\subsubsection{Spontaneous fission}

Smolańczuk et al. [Smolańczuk 1995] have made predictions of the SF halflives for even-even isotopes near the $\mathrm{N}=162$ deformed shell. The predicted half-lives for nuclides neighboring the two bohrium isotopes at $\mathrm{Z}=108$ and 106 , and $\mathrm{N}=158$ and 160 are: 1.6 and $96 \mathrm{~s}$, for ${ }^{266} \mathrm{Hs}$ and ${ }^{268} \mathrm{Hs}$, and 2.3 and $58 \mathrm{~s}$ for ${ }^{264} \mathrm{Sg}$ and ${ }^{266} \mathrm{Sg}$. The only isotopes in the region for which there are experimental SF data are ${ }^{262} \mathrm{Rf},{ }^{263} \mathrm{Ha}$, and ${ }^{266} \mathrm{Sg}$, with SF half-lives of $2.1 \mathrm{~s}$ [Lane 1996], $>47$ s, and $>11 \mathrm{~s}$ [Türler 1998a], respectively. The experimentally measured partial SF half-lives are in line with the theoretical predictions. Since the bohrium nuclides addressed by this experiment will have odd-particle hindrance factors against SF on the order of $10^{5}$, we expect that SF will not be the predominant decay mode.

\subsubsection{Alpha decay}

The calculations of Smolańczuk et al. reproduce well the experimentally observed heavy element $\alpha$-decay energies. Extrapolating from their calculations for even-even nuclei [Smolańczuk 1997], it is possible to obtain $Q_{\alpha}$ values for the odd-Z bohrium isotopes. Using these Smolańczuk $Q_{\alpha}$ values, together with the $\alpha$-half-life systematics of Hatsukawa [Hatsukawa 1990] results in the following (using an $\alpha$ -

decay hindrance factor of 2.0 for odd-even ${ }^{267} \mathrm{Bh}$ and 5.0 for odd-odd ${ }^{266} \mathrm{Bh}$ ) predictions: 


$$
\begin{aligned}
& { }^{267} \mathrm{Bh} \rightarrow{ }^{263} \mathrm{Db}+\alpha, \mathrm{E}_{\alpha}=9.0 \mathrm{MeV}, \mathrm{t}_{1 / 2(\mathrm{HF}=0)}=2.1 \mathrm{~s}, \mathrm{t}_{1 / 2(\mathrm{HF}=2)}=4.2 \mathrm{~s} \\
& { }^{266} \mathrm{Bh} \rightarrow{ }^{262} \mathrm{Db}+\alpha, \mathrm{E}_{\alpha}=9.25 \mathrm{MeV}, \mathrm{t}_{1 / 2(\mathrm{HF}=0)}=0.4 \mathrm{~s}, \mathrm{t}_{1 / 2(\mathrm{HF}=5)}=2.0 \mathrm{~s}
\end{aligned}
$$

During an experiment that will be described later, the half-life and $\alpha$-particle energy of ${ }^{267} \mathrm{Bh}$ was measured to be $17_{-6}^{+14} \mathrm{~s}$ and $8.83 \pm 0.03 \mathrm{MeV}$. The longer half-life corresponds to the lower $\alpha$-decay energy, and agrees well with Hatsukawa systematics. The one 9.27-MeV $\alpha$-particle attributed to ${ }^{266} \mathrm{Bh}$ in this experiment is what is expected, and its lifetime also is consistent with Hatsukawa systematics.

\subsubsection{Electron-capture decay}

Two recent mass formulations exist for calculation of heavy element Q-values. The compilation of Möller et al. [Möller 1996] uses a Thomas-Fermi macroscopic part together with Möller unique-particle shell corrections. This compilation has had trouble reproducing experimental decay energies in the heavy element region. The model of Smolańczuk et al. [Smolańczuk 1997] is much better at reproducing the experimentally observed heavy element $\alpha$-particles energies. While Smolańczuk does not give the total mass, good success in reproducing decay energies can be obtained by using the macroscopic masses from Möller, and adding the shell correction from Smolańczuk. Using these modified masses, together with an assumed $\log (\mathrm{ft})$ of 5.0, the electron-capture (EC) decay half-lives for ${ }^{267} \mathrm{Bh}$ and ${ }^{266} \mathrm{Bh}$ are:

$$
\begin{aligned}
& { }^{267} \mathrm{Bh} \rightarrow{ }^{267} \mathrm{Sg}, \mathrm{Q}_{\mathrm{EC}}=3.47 \mathrm{MeV}, \mathrm{t}_{1 / 2(\log (\mathrm{ft})=5.0)}=50 \mathrm{~s} \\
& { }^{266} \mathrm{Bh} \rightarrow{ }^{266} \mathrm{Sg}, \mathrm{Q}_{\mathrm{EC}}=5.27 \mathrm{MeV}, \mathrm{t}_{1 / 2(\log (\mathrm{ft})=5.0)}=20 \mathrm{~s}
\end{aligned}
$$

These should be considered lower limits, since a relatively low $\log (\mathrm{ft})$ was 
used, and decay directly to the ground-state of the daughter was assumed. Taken together, these calculations suggest that for both bohrium isotopes, $\alpha$-decay with halflives of ten seconds or more could be expected.

\subsection{Chemical Theory}

\subsubsection{Importance of theory to the experimentalist}

Just as investigation of chemical properties of the heaviest elements goes handin-hand with the investigation of their nuclear properties, so does theory accompany experiment. Due to the increasingly small cross sections of the heaviest elements, the experiments to determine their chemical properties are more and more costly, as well as increasingly complex. The small production cross sections require long periods of accelerator time for these experiments from multi-user facilities where competition for the beam time is fierce. These factors make predictive calculations an integral part of the scientific process for the investigation of the chemical properties of the heaviest elements. Likewise symbiotically, experimental results are also needed by the theoreticians to develop and test their theories. The close relationship between experimentation and calculation allows the theoreticians to refine and update their theories. In addition, analytical theory can sometimes give insight into chemical processes.

\subsubsection{Relativistic calculations}

As elements get heavier and their nuclear charge increases, the binding energy 
of the inner elections also increases. These inner electrons in heavy elements are so tightly bound that they are accelerated to velocities that are a significant fraction of the speed of light. The ways in which chemical properties are changed by these relativistically accelerated electrons, are referred to as relativistic effects. Two general relativistic effects inferred from the solving of the relativistic Shroedinger equation called the Dirac equation - are the stabilization and contraction of the $s$ and $p_{\frac{1}{2}}$ orbitals and the subsequent destabilization and expansion of the $d$ orbitals due to increased screening by the inner orbitals. Curiously, unlike the non-relativistic solution, the $p_{1 / 2}$ atomic orbital is spherical like the $s$. In addition to the relativistic effect causing changes in atomic radii and degree of covalency in molecules, the radial changes in the size of the $d$-orbitals will have an effect on molecular bonding.

Since chemical properties are dictated by electronic structure and relativistic effects should increase as a function of $\mathrm{Z}^{2}$, traditional extrapolation of periodic properties down and across the periodic table is expected to be misleading at some point. Where the relativistic effects become important as well as the extent of these effects, is currently an area of some contention. For example, the relativistic effect is already important in gold; in the absence of the relativistic effect, gold would be expected to have the same color as silver! [Pyykkö 1988]. The actinide series contract as one progresses down the series analogous to the lanthanides, except in the actinides, the contraction is caused by relativity. This contraction will even continue past the end of the actinide series, with element 111 having an atomic radius smaller than gold [Seth 1995]. The problem in predicting chemical behavior lies with the extreme difficulty presented by trying to do calculations involving many electrons. However, 
the processing power made available with current computers has allowed theoreticians to make reasonable predictions concerning the chemical properties of molecules formed with group five and seven heavy elements. The calculations of Pershina et al. as they pertain to our experiments will be discussed in the next sections. For more than 10 years, Valeria Pershina and her co-authors have been doing theoretical research on the heavy elements and they provide increasingly more accurate calculations as they make steady progress in relativistic density functional theory (for example: [Varga 2000]). To date, their calculations have not been surpassed in predictive value for the experimentalist.

\subsubsection{Rutherfordium}

Rutherfordium, element 104, is the first transactinide element. As such, it is expected to be a group four element and thus behave like zirconium, hafnium, and titanium. Zirconium and hafnium have very similar chemical properties which are attributed to their similar electronic structure as reflected by their nearly identical atomic and ionic radii. Pershina et al. calculate that the radii of rutherfordium should be similar to zirconium and hafnium, and not titanium [Pershina 1996a]. Thus, it is expected from this simple extrapolation that the chemistry of rutherfordium is more similar to zirconium and hafnium, than to that of titanium. This has been verified experimentally [Schädel 1995b]. Rutherfordium metal is expected have the electronic structure $6 d^{3} 7 s$ by analogy to its homologues.

However in the gas phase, rutherfordium with a $6 d^{2} 7 s^{2}$ configuration is expected to have a higher energy of sublimation than rutherfordium with a $6 d^{1} 7 s^{2} 7 p$ 
configuration (or $6 d^{3} 7 s$ assumably), according to calculations by Zhuikov et al. [Zhuikov 1990]. They attempted an experiment [Zhuikov 1990] to assess the $p$ or $d$ nature of rutherfordium in the gas phase. While they were unable to definitively assess the electronic structure of rutherfordium in the gas phase, they were able to conclude that the sublimation enthalpy $\left(\Delta \mathrm{H}_{\mathrm{s}}\right)$ is much higher than that of lead, which indicates it is not a $p$-element analogous to lead. While rutherfordium may have a $p$ valence orbital in the gas phase, lead has completely filled $d$-orbitals and a large energy gap to the first excited electronic state. However, the actual electronic structure of rutherfordium, whether it be $6 d^{2} 7 s^{2}, 6 d^{1} 7 s^{2} 7 p_{1 / 2}$ or even $6 d^{2} 7 p^{2}$ (unlikely) is still unresolved [Pershina 1996a].

Pershina reports that everybody is currently in agreement that the electronic structure of $\mathrm{RfCl}_{4}$ is similar to $\mathrm{HfCl}_{4}$ and not $\mathrm{PbCl}_{4}$ [Pershina 1996a]. Beyond this, however, the predictions for the gas chemistry of rutherfordium halides have not always been in agreement. Based on their calculations of ionization potentials, Zhuikov et al. predicted that the lower halides of rutherfordium would be more stable and less volatile than those of zirconium and hafnium [Zhuikov 1990], which is the same as one would expect from simple extrapolation down the group from periodic trends. This is opposite of what has been calculated by Pershina et al. [Pershina 1996a] and others [Malli 1998], and what has been observed experimentally [Sylwester 1998]. Zhuikov et al. speculate that this might be due to differences in covalency between the halides of hafnium and rutherfordium [Zhuikov 1990]. This seems to agree with the calculations of Pershina et al. who find that $\mathrm{RuCl}_{4}$ is expected to be the most covalent halide of the group and thus the most volatile [Pershina 
1996a]. This volatility has been verified experimentally, with $\mathrm{RfCl}_{4}$ being more volatile than $\mathrm{HfCl}_{4}$. In addition, the volatility of the bromides has been measured, and the same ordering is seen: $\mathrm{RfBr}_{4} \geq \mathrm{ZrBr}_{4}>\mathrm{HfBr}_{4}$. The bromide species were slightly less volatile than the chloride species, as expected by theory [Sylwester 1998]. The first volatility experiments with rutherfordium were attempted by Zvára et al. beginning in 1966, but were inconclusive [Zvára 1971, Hyde 1987].

Türler et al., while verifying the volatility results of rutherfordium chloride, found that the introduction of oxygen into the experimental apparatus shifted the volatilities of rutherfordium and hafnium chlorides about $200^{\circ} \mathrm{C}$ higher. Hafnium oxychloride and rutherfordium oxychloride are not expected to be stable in the gas phase [Türler 1998b]. Unfortunately there have been no calculations concerning the relative properties of the group four oxyhalides, but Türler et al. cite a study by Morozov et al. in which they found that zirconium and hafnium oxychloride decompose when heated [Morozov 1971]. By analogy with the group five and six elements, the oxychlorides should be more stable to decomposition [Pershina 1996a] and would be expected to be more volatile than the pure halides. However, Türler et al. conclude that this is a curious result that seems to indicate that the "oxychloride exists in the condensed phase and not the gas phase", and can influence transport of the group four elements through the chromatography column [Türler 1998b].

Rutherfordium has been observed to elute from cation exchange resin with dilute $\mathrm{HCl} / \mathrm{HF}$ [Schumann 1998] and $\mathrm{HNO}_{3} / \mathrm{HF}$ [Strub 2001] in a manner more similar to its pseudo-homologue thorium than zirconium or hafnium. The order of stability of the complexes in these solutions has been measured to be $\mathrm{Zr}>\mathrm{Hf} \gg \mathrm{Rf} \geq \mathrm{Th}$. This is very 
analogous to the group five behavior in dilute $\mathrm{HCl} / \mathrm{HF}$ (see section 2.2.4).

Experiments in pure $\mathrm{HCl}$ need to be done (see Chapter 6:Conclusions and Future Work).

\subsubsection{Hahnium}

Periodic trends indicate that hahnium should behave similarly to its group five homologues niobium and tantalum, following trends down the group starting with vanadium. Protactinium will be included in this discussion as it is considered to be a group five pseudo-homologue. It shares a 5+ oxidation state with the group five elements, as well as other similar properties such as a tendency to form polymers, tendency to be adsorbed onto any available surface, and it is never seen as an uncoordinated cation due to its strong tendency to undergo hydrolysis [Kirby 1959, Kirby 1986]. These similarities are due to their analogous electronic structures.

Pershina et al. predict hahnium has a electronic structure analogous to vanadium's $3 d^{3} 4 s^{2}$ and tantalum's $5 d^{3} 6 s^{2}$, while niobium has a $4 d^{4} 5 s^{1}$ structure and protactinium has a $5 f^{2} 6 d^{1} 7 s^{2}$ structure (see Table 2.1) [Pershina 1992a]. Earlier predictions of the electronic structure included the possibility of a stabilized $p_{\frac{1}{2}}$ orbital giving a structure of $5 d^{2} 7 s^{2} 7 p_{1 / 2}$.

Table 2.1 Electronic ground state structure of the group five elements

\begin{tabular}{cc}
\hline Element (ground state) & Electronic Configuration \\
\hline vanadium $(\mathrm{V})$ & {$[\mathrm{Ar}] 3 d^{3} 4 s^{2}$} \\
niobium $(\mathrm{Nb})$ & {$[\mathrm{Kr}] 4 d^{4} 5 s^{1}$} \\
tantalum $(\mathrm{Ta})$ & {$[\mathrm{Xe}] 4 f^{4} 5 d^{3} 6 s^{2}$} \\
hahnium $(\mathrm{Ha})$ & {$[\mathrm{Rn}] 5 f^{44} 6 d^{3} 7 s^{2}$} \\
protactinium $(\mathrm{Pa})$ & {$[\mathrm{Rn}] 5 f^{2} 6 d^{1} 7 s^{2}$} \\
\hline
\end{tabular}


Niobium and tantalum, as well as protactinium, form stable pentahalides and oxyhalides. All of the pentahalides of the group five elements are known, and both the bromide and chloride oxyhalides of niobium and tantalum are known [Steinberg 1961, Türler 1999]. Pershina and co-workers calculated that the covalency, stability of bonding, and the stability of the maximum $5+$ valence state should increase down the group, while the $3+$ and the $4+$ valence state should decrease down the group. This is opposite to what one would expect in going from tantalum $(5 d)$ to hahnium $(6 d)$ and not taking relativity into account [Pershina 1992a, Pershina 1993]. Earlier predictions postulated that if the $7 s$ orbitals were sufficiently stabilized due to relativistic effects, it is possible that hahnium would have a $3+$ valence state $[\mathrm{Rn}] 5 \mathrm{f}^{14} 7 s^{2}$ in solution [Hoffman 1994].

The oxychlorides are predicted to be less volatile than the corresponding pentahalides and the bromides should be less volatile than the chlorides [Pershina 1992c, Pershina 1996]. This has been verified experimentally [Türler 1996, Sylwester 1998]. From theoretical interpretations, Pershina et al. hypothesize that $\mathrm{HaBr}_{5}$ should be more volatile than $\mathrm{NbBr}_{5}$ and $\mathrm{TaBr}_{5}$ [Pershina 1992b], though it should be noted that Dr. Pershina does not mention this prediction in later papers. There are not really any predictions on the relative volatilities down the group for these halides and oxyhalides other than that. The experimental evidence seems to contradict this prediction, with hahnium halides being less volatile than those of niobium [Zvára 1976, Schädel 1995a, Sylwester 1998]. Türler et al. also found experimentally that $\mathrm{HaOCl}_{3}$ has a lower volatility than $\mathrm{NbOCl}_{3}$ [Türler 1996]. However, there is some contention that when measuring the pure halides there was some oxygen 
contamination which skewed the data [Schädel 1995a] and the Pershina prediction might still be correct. The group five elements are extremely reactive with even very small amounts of oxygen.

Pershina et al. performed calculations on the anionic complexes $\left[\mathrm{MCl}_{6}\right]^{-}$, $\left[\mathrm{MOCl}^{4}\right]^{-},\left[\mathrm{M}(\mathrm{OH})_{2} \mathrm{Cl}_{4}\right]^{-},\left[\mathrm{MOCl}_{5}\right]^{2-}$ where $\mathrm{M}=\mathrm{Nb}, \mathrm{Ta}, \mathrm{Ha}$, or Pa. They found niobium and hahnium form the hexahalide $\left[\mathrm{MCl}_{6}\right]^{-}$and the oxyhalide $\left[\mathrm{MOCl}_{5}\right]^{2-}$ with about equal probability; however, at medium acid concentrations there will be a tendency for hahnium to form the oxyhalide $\left[\mathrm{HaOCl}_{5}\right]^{2-}$. Protactinium is also expected to preferentially form the oxyhalide $\left[\mathrm{PaOCl}_{5}\right]^{2-}$, except at high acid concentrations. At high $\mathrm{HCl}$ concentrations protactinium as well as hahnium is expected to form the pure halide $\left[\mathrm{Pa} \text { or } \mathrm{HaCl}_{7}\right]^{2-}$. They also found that tantalum has a tendency to form the pure hexahalide $\left[\mathrm{TaCl}_{6}\right]^{2-}[$ Pershina 1994]. Tantalum's special place in the group is thought to be due to it being at a position in the group where "some effects are compensated by the others"; i.e., where the relativistic effects, which are expected to decrease the radius, are counterbalanced by the radial expansion expected due to the addition of another electronic shell. For example, there is no change in ionic radii from $\mathrm{Nb}$ to $\mathrm{Ta}$, which is also seen in $\mathrm{Zr} / \mathrm{Hf}$ and Mo/W [Pershina 1992a]. Pershina calculated that the tendency to undergo hydrolysis in aqueous acidic solutions decreases in the order $\mathrm{Nb}>\mathrm{Ta}>\mathrm{Ha} \gg \mathrm{Pa}$ in $\mathrm{HCl}$ [Pershina 1998a], or $\mathrm{Ta}>\mathrm{Ha}>\mathrm{Nb}>\mathrm{Pa}$ in $\mathrm{HF}$ and $\mathrm{HBr}$ solutions. In $\mathrm{HF}$ and $\mathrm{HBr}$ solution the sequence of extractability is predicted to be $\mathrm{Pa} \gg \mathrm{Nb} \geq \mathrm{Ha}>\mathrm{Ta}$ [Pershina 1999b]. Pershina calculates that the trend of extractability above $4 \mathrm{M} \mathrm{HCl}$ into an organic phase should be $\mathrm{Pa} \gg \mathrm{Nb} \geq \mathrm{Ha}>\mathrm{Ta}$ [Pershina 1998b]. Previously, Pershina et al. predicted, based on the size of the complexed ions, that 
protactinium should have a higher partition coefficient than niobium, with hahnium in the middle between the two [Pershina 1994].

Experimentally, hahnium exhibits curious solution chemistry. In pure $\mathrm{HCl}$, hahnium extracts into an organic phase most like niobium, and not at all like protactinium. Hahnium's behavior in pure 4M HF is similar, it extracts like niobium and tantalum and not like protactinium. However, in a mixed aqueous phase of $\mathrm{HCl}$ and dilute $\mathrm{HF}$, hahnium extracts like protactinium and differs greatly from the behavior of tantalum [Paulus 1998, Paulus 1999]. This is not unlike the behavior observed for the group four elements (see section 2.2.3 ).

\subsubsection{Seaborgium}

Seaborgium is expected to have the same electronic structure as tungsten, differing from the lighter homologues chromium and molybdenum (see Table 2.2). This electronic structure with the increased $s$-character for the heavier elements is the result of relativistic effects [Johnson 1999].

Table 2.2 Electronic ground state structure of the group six elements

\begin{tabular}{cc}
\hline Element (ground state) & Electronic Configuration \\
\hline Chromium $(\mathrm{Cr})$ & {$[\mathrm{Rn}] 3 d^{5} 4 s^{1}$} \\
Molybdenum $(\mathrm{Mo})$ & {$[\mathrm{Kr}] 4 d^{5} 5 s^{1}$} \\
Tungsten $(\mathrm{W})$ & {$[\mathrm{Xe}] 4 f^{4} 5 d^{4} 6 s^{2}$} \\
Seaborgium $(\mathrm{Sg})$ & {$[\mathrm{Rn}] 5 f^{14} 6 d^{4} 7 s^{2}$} \\
\hline
\end{tabular}

Seaborgium is expected to still prefer a $6^{+}$oxidation state like molybdenum and tungsten, but there is a trend across the period from $\mathrm{RfCl}_{4}$ to $\mathrm{SgCl}_{6}$ of a decrease in stability of the maximum oxidation state. Chromium has common oxidation states $2+$, $3+$, and $6+$ with the $3+$ state being most stable. Going down the group, Pershina et al. 
expect the stability of the $6^{+}$oxidation state to increase from chromium to seaborgium $(\mathrm{Cr}<\mathrm{Mo}<\mathrm{W}<\mathrm{Sg})$, while the relative stability of the $5+, 4+$, and $3+$ decreases down the group [Pershina 1999a], which is analogous to the trend predicted for the group five elements. While seaborgium does not have a preference for the $4+$ oxidation state, it is expected that it will be easier to reduce $\mathrm{Sg}^{6+}$ to $\mathrm{Sg}^{4+}$ than to $\mathrm{Sg}^{3+}$ even though it is even harder to reduce $\mathrm{Sg}^{6+}$ to $\mathrm{Sg}^{4+}$ than to reduce $\mathrm{W}^{6+}$ to $\mathrm{W}^{4+}$ due to the relative stability of the $4+$ state with respect to the $3+$ state increases as you go down the group [Pershina 1999a]. There had been some speculation that the $7 s^{2}$ orbitals would be greatly relativistically stabilized in $\mathrm{Sg}^{4+}$, but this seems not to be the case. Based on systematics Eichler et al. predict that the stability of the group six dichlorides, tetrachlorides, and the hexachlorides increases down the group while the trichloride is decreasing and the pentachloride remains the same [Türler 1999]. This seems to agree well with the calculations of Pershina.

The dioxydichlorides should be less volatile than the oxytetrachlorides. This is due to less covalency and a larger dipole of the dioxydichloride [Pershina 1996b]. This is fortunate as the oxytetrachlorides of the group 6 elements are fairly unstable. Gas chemistry of seaborgium oxytetrachloride would be ambiguous due to uncertainty of actual chemical structure, $\mathrm{SgOCl}_{4}$ or $\mathrm{SgOCl}_{3}$, because $\mathrm{SgOCl}_{4}$ 's stability to decomposition is approximately equal to that of $\mathrm{MoOCl}_{4}$, which decomposes at room temperature [Pershina 1995]. Pershina et al. predict the volatility of seaborgium dioxydichloride to be less than that of the other group six elements [Pershina 1996a], which is what has been observed experimentally. Türler writes that based on the experiments that he and his colleagues have performed "The probability that $\mathrm{SgO}_{2} \mathrm{Cl}_{2}$ 
is equally or even more volatile than $\mathrm{MoO}_{2} \mathrm{Cl}_{2}$ was estimated to be less than $15 \%$ " with the volatility order reported as $\mathrm{MoO}_{2} \mathrm{Cl}_{2}>\mathrm{WO}_{2} \mathrm{Cl}_{2} \approx \mathrm{SgO}_{2} \mathrm{Cl}_{2}$ [Türler 1999]. Hydrolysis from the cationic species to the neutral species decreases in the order: $\mathrm{Mo}>\mathrm{W}>\mathrm{Sg}$. However, continuing the hydrolysis to the formation of the anionic oxo-complexes, the order changes to $\mathrm{Mo}>\mathrm{Sg}>\mathrm{W}$ [Pershina 2000b]. This is the same behavior of hydrolysis and complexation observed in the group five elements. In basic, aqueous solution, the group six elements chromium, molybdenum, and tungsten form tetraoxyanions like $\left[\mathrm{CrO}_{4}\right]^{2-}$. Seaborgium is expected to form the seaborgate $\left[\mathrm{SgO}_{4}\right]^{2-}$ in solution. However, its stability should be the least in the group, although its $6+$ oxidation state is the most stable of the group in solution. [Pershina 1996a]. In acidic solutions the group six elements form a variety of oxyhalide ions ranging from the dioxydihalide to the dioxytetrahalide. These sometimes exist depending on the acid concentration as the water adduct. In organic solutions, the water adduct can be replaced with the solvent molecule. As in the group five elements, extraction of the group six from $\mathrm{HCl}$ into an organic phase depends on the relative dipole moment of the molecule [Pershina 1996a]. Uranium has significantly different extraction properties than the other group six elements due to its linear structure in solution (a result of its $f d$ hybridization) and hence no dipole. Seaborgium is expected to behave like the other group six elements (a $d s$ hybrid resulting in a bent structure) and not like its pseudo-homologue uranium [Pershina 1996a]. In solutions with $\mathrm{HF}$ and $\mathrm{HCl}$, the group six elements form oxyfluorides or oxychlorides, depending on the relative concentration of each acid. The presence of HF in an acid solution will strongly affect its extraction behavior. 
The experimental results of Schädel et al. confirm these predictions. They found that seaborgium is eluted from an anion exchange column using dilute $0.1 \mathrm{M}$ $\mathrm{HNO}_{3} / 5 \times 10^{-4} \mathrm{M} \mathrm{HF}$ in a manner analogous to molybdenum and tungsten, and not like uranium which is retained on the column [Schädel 1997, Schädel 1998, Lougheed 1997b]. Furthermore, they found that when using only dilute $\mathrm{HNO}_{3}(0.1 \mathrm{M})$, the seaborgium is not eluted, which indicates that even under very dilute HF condition the fluoride ions play a very active role in complexation in solution [Schädel 1998].

While mostly only the oxygen and halide containing heavy elements have been studied by theory and experiment, there are many other interesting compounds that

\section{Figure 2.1 Photolysis of chromium hexacarbonyl followed by the formation of the solvated chromium pentacarbonyl species.}

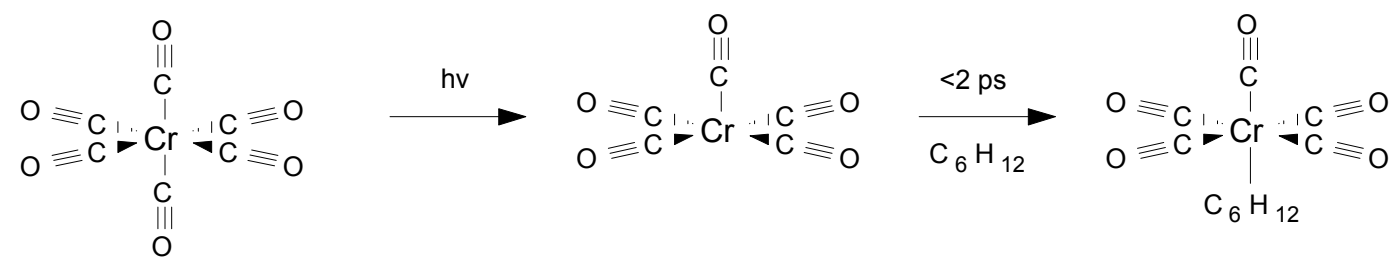

could be studied. One group of compounds that I find particularly interesting is the group six carbonyls. Before coming to Berkeley, I studied using time-resolved resonance Raman spectroscopy to study chromium pentacarbonyl, which is formed by using a separate pump laser to disassociate a carbonyl from the hexacarbonyl [Wilk 1995]. The chromium pentacarbonyl is a very reactive species that has interesting properties, such as its ability to form weak bonds with solvent molecules (see Figure 2.1). Appropriately enough, Pershina reports that the group six carbonyl of seaborgium hexacarbonyl has also been studied theoretically [Pershina 1996, Nash 1995]. The hexacarbonyl of seaborgium is expected to be similar to that of chromium 
and tungsten with "significant $n \mathrm{~d}(\mathrm{M})-\pi(\mathrm{CO})$ back-bonding", and unlike that of $\mathrm{U}(\mathrm{CO})_{6}$ due to uranium's $d$ and $f$ valence electrons. The $\mathrm{t}_{2 \mathrm{~g}}$ highest occupied molecular orbital (HOMO) is calculated to be lowered to the level of the analogous orbital in $\mathrm{Cr}(\mathrm{CO})_{6}$. It is likely that $\mathrm{Sg}(\mathrm{CO})_{6}$ could be studied as an isolatable species.

\subsubsection{Bohrium}

Bohrium is expected to be a group seven element and should form oxychloride species in the gas phase in an analogous fashion to rhenium and technetium. The $\mathrm{MO}_{3} \mathrm{Cl}(\mathrm{M}$ being $\mathrm{Tc}, \mathrm{Re}$, or $\mathrm{Bh})$ species is the most stable and volatile, although Tc and $\mathrm{Re}$ are also known to form $\mathrm{TcOCl}_{3}, \mathrm{TcOCl}_{4}$, and $\mathrm{ReOCl}_{4}$ [Colton 1965]. During test experiments with rhenium and technetium [Eichler 1999, Eichler 2000d], only one chemical species was seen with the thermochromatography apparatus (described in section 5.2.1 ). This species was assumed to be $\mathrm{MO}_{3} \mathrm{Cl}$ and Pershina et al. only performed calculations on this species [Pershina 2000a].

They found that the electronic structure of $\mathrm{BhO}_{3} \mathrm{Cl}$ is very similar to $\mathrm{TcO}_{3} \mathrm{Cl}$ and $\mathrm{ReO}_{3} \mathrm{Cl}$. The calculated energy gap $(\Delta E)$ and ionization potential (IP) increase from Tc to $\mathrm{Bh}$ for the $\mathrm{MO}_{3} \mathrm{Cl}$ species $(\Delta E: 2.83,3.21,3.26 \mathrm{eV}$ and IP: $12.25,12.71,13.05$ $\mathrm{eV}$ ). The 7+ oxidation state should also increase in stability down the group following the increasing $\Delta E$. The Bh species is also more covalent than the other two species which they attribute to relativistic effects seen in the component atomic orbital. These effects also influence the dipole moments and the dipole polarizabilities calculated, which in turn dictate intermolecular or molecule-surface interactions. These interactions, they conclude, are the basis for volatility that they predict should 
decrease down the group, i.e. that $\mathrm{BhO}_{3} \mathrm{Cl}$ should be less volatile than $\mathrm{ReO}_{3} \mathrm{Cl}$ which should be less volatile than $\mathrm{TcO}_{3} \mathrm{Cl}$. In terms of adsorption enthalpy, they predict $\Delta H_{\mathrm{abs}}\left(\mathrm{TcO}_{3} \mathrm{Cl}\right)=-48 \pm 2$ and $\Delta H_{\mathrm{abs}}\left(\mathrm{BhO}_{3} \mathrm{Cl}\right)=-78 \mathrm{~kJ} / \mathrm{mol}$. In addition, they calculated the decomposition energy of $\mathrm{BhO}_{3} \mathrm{Cl}$, and although it is less than that for the Tc and $\mathrm{Re}$ species, it is still high enough that the Bh species should be expected to be stable at experimental temperatures around $100{ }^{\circ} \mathrm{C}$ [Pershina 2000a].

Pershina et al. have shown that the stability of the maximum oxidation state decreases across the $6 d$ series based on their calculations on rutherfordium, hahnium, and seaborgium. They expect that bohrium, and elements beyond, will prefer lower oxidation states in aqueous solution [Pershina 1999a]. 


\section{Chapter 3: Instrumentation and Techniques}

The apparatus and techniques used in the experiments to determine the nuclear properties of ${ }^{224} \mathrm{~Pa},{ }^{266} \mathrm{Bh}$, and ${ }^{267} \mathrm{Bh}$ were all very similar. While it is sometimes necessary to use a chemical separation to remove reaction products that would mask the detection of the isotopes of interest, it was not necessary in this case. Although the nuclei of interest were only a tiny fraction of the products produced in the bombardment of the targets, it was possible to remove the effects of other products by judicious use of detector gates and very careful preparation of clean targets. The

following reactions were used in our studies: ${ }^{224} \mathrm{~Pa}$ was produced via the ${ }^{209} \mathrm{Bi}\left({ }^{18} \mathrm{O}, 3 n\right)$ reaction and ${ }^{266} \mathrm{Bh}$ and ${ }^{267} \mathrm{Bh}$ via the ${ }^{249} \mathrm{Bk}\left({ }^{22} \mathrm{Ne}, 5 n\right)$ and ${ }^{249} \mathrm{Bk}\left({ }^{22} \mathrm{Ne}, 4 n\right)$ reactions.

Since the need for chemical separation was removed, the apparatus required consisted of six basic parts: the accelerator, the target chamber, the transport system, the collection system, the detector array, and the data acquisition system.

\subsection{Accelerator}

The Lawrence Berkeley National Laboratory (LBNL) 88-Inch Cyclotron provided high intensity beams of ${ }^{18} \mathrm{O}$ and ${ }^{22} \mathrm{Ne}$ for the experiments. The LBNL cyclotron is one of the premier facilities in the world for providing beams of high intensity light to medium weight-ions and is capable of accelerating ions from hydrogen to uranium. Through ECR (Electron Cyclotron Resonance) ion-source development, the 88-Inch Cyclotron has been able to keep in the forefront of accelerator capability in spite of its advanced age - it was built in 1961. Most similar 
facilities use variations of the ECR ion-source that was developed here. Due in large part to this technology, the cyclotron can produce a wide variety of relatively intense and reliable beams.

After the ions are initially produced, accelerated, and directed down the beam line toward our experimental facility, the beam then enters our target chamber. The cyclotron is operated by a staff committed solely to running and maintaining this accelerator.

\subsection{Target Chamber and Targets}

The target chamber [see Figure 3.1] was separated from the beam line by a $2.73-\mathrm{mg} / \mathrm{cm}^{2}$ beryllium vacuum window. After passing through this window, the beam then passes through $0.5 \mathrm{mg} / \mathrm{cm}^{2}$ of nitrogen cooling gas before hitting the target and before hitting the target-backing, if one is required. In addition to gas cooling of the

\section{Figure 3.1 Collection and Transport System}

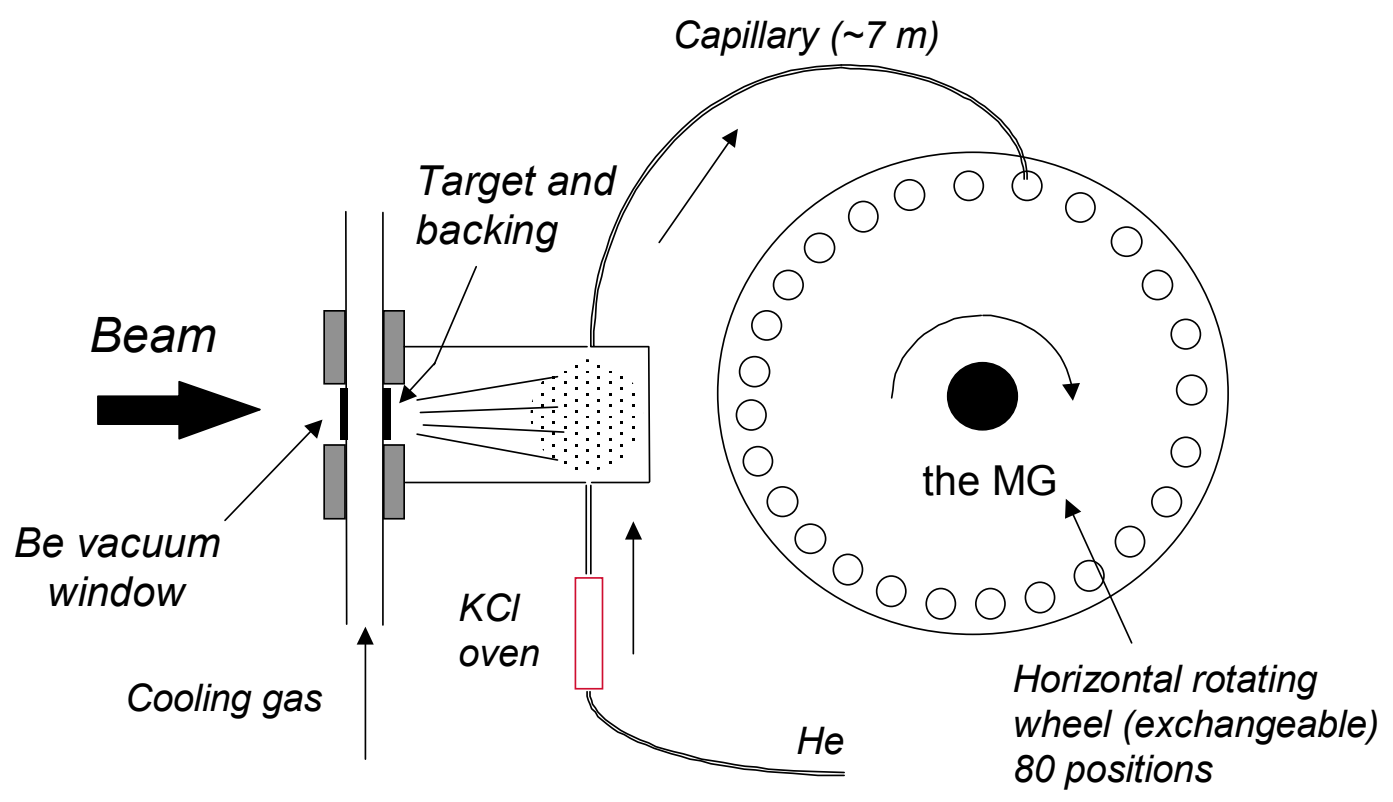


target, a beam wobbler is used to disperse the beam over the target.

\subsubsection{Bismuth Target}

The $3.3 \mathrm{mg} / \mathrm{cm}^{2}$ of ${ }^{209} \mathrm{Bi}$ target material was electroplated in a diameter of $6 \mathrm{~mm}$ on a $2.35-\mathrm{mg} / \mathrm{cm}^{2}$ beryllium target backing [Aumann 1974]. This target is thick compared to the recoil range of the reaction products which recoil only out of approximately the last half of the target at the bombarding energy used.

\subsubsection{Berkelium Target}

The berkelium target material was provided by the transplutonium element production program at the Oak Ridge National Laboratory. Two sets of two targets were prepared, one in March 1999 and one in October of 2000. Due to the relatively short 320-day half-life of ${ }^{249} \mathrm{Bk}$ and the in-growth of its ${ }^{249} \mathrm{Cf}$ daughter, it is necessary to purify and re-fabricate newly separated targets before each of the experiments.

The ${ }^{249} \mathrm{Cf}$ daughter $\left(\mathrm{T}_{1 / 2}=351\right.$ years $)$ was separated from the ${ }^{249} \mathrm{Bk}$ target material ( $T_{1 / 2}=320$ days) five days prior to the beginning of the experiment with less than 0.5 atom $\%{ }^{249} \mathrm{Cf}$ present after separation. The target material was then deposited on a $2.73-\mathrm{mg} / \mathrm{cm}^{2}$ beryllium foil by the molecular plating technique. Targets prepared by this technique are able to withstand the harsh conditions that the targets are subjected to in the target chamber without loss of target material.

High voltage electrodeposition of the nitrate in an isopropanol solution is used to plate the target material on the beryllium backing. A 6-mm diameter cylindrical chimney is placed on top of the beryllium foil, which is held on an aluminum base plate. This beryllium foil acts as the cathode and a platinum electrode placed midway 
in the cylinder is the anode. The solution is placed in the cell and a spinning glass rod is used to agitate the solution. Several hundred volts are applied across the cell for about 30 minutes, after which the target is placed in an oven at $550{ }^{\circ} \mathrm{C}$ to convert the material to the oxide. The target is then placed back beneath the cell and the process is repeated until the target reaches the desired thickness. The target thickness is determined by measuring the $\alpha$-activity of the target in a known geometry [Evans 1972, Aumann 1975, Müllen 1975].

Due to the safety issues inherent in working with actinide materials, it is necessary for the work to be done in a dedicated glove box that is used only for actinide target making. There are significant bureaucratic hurdles that must be overcome to dispose of the small amount of waste that is generated during the target making process. A considerable amount of time must be devoted to such issues before target fabrication can commence. In addition to this, much thought, training, and planning takes place before target fabrication to ensure a minimum of exposure to the chemists, and to maintain the highest possible level of safety.

Once items are used in the dedicated glove box, they are either left inside for the next target fabrication, or they are disposed of - there is no decontamination possible. This also makes repair and retrofitting of the glove box very difficult. The $\alpha$-detector that is used in the glove box is a small solid angle detector mounted on the target preparation glove box and operated under helium. Operating the detector under helium alleviated the need for a vacuum set-up in the glove box. The range of an $\alpha$-particle in helium is about four times farther than in air.

Even though the target material adheres well to the target backing, a very thin 
$50-\mu \mathrm{g} / \mathrm{cm}^{2} \mathrm{Al}$ cover foil was placed over the target to prevent contamination from recoiling atoms from decay of the radioactive target or any sputtering of target material by the cyclotron beam. This target was then used in the subsequent bohrium chemistry experiments at the Paul Scherrer Institut in Switzerland (PSI).

The first set of targets was prepared by Dr. Carola Laue while the second set of two targets was prepared by Dr. Ralph Sudowe. Both target sets were prepared in an identical fashion and were also used subsequently in our chemistry experiment at PSI after first being used at LBNL for my experiments to determine the decay properties of bohrium. In both cases only the first target of the set was used, and the second was kept as a backup. The first target used was $810 \mu \mathrm{g} / \mathrm{cm}^{2}$ and the second target used was $478 \mu \mathrm{g} / \mathrm{cm}^{2}$, both as the oxide. After use at LBNL, a layer of terbium was added so that rhenium would be made online via the ${ }^{159} \mathrm{~Tb}\left({ }^{22} \mathrm{Ne}, 5 n\right){ }^{176} \mathrm{Re}$, concurrent with bohrium for direct comparison with the bohrium in the chemical experiments.

\subsection{Production Reactions}

3.3.1 The ${ }^{209} \mathrm{Bi}\left({ }^{18} \mathrm{O}, 3 n\right){ }^{224} \mathrm{~Pa}$ reaction

In our experiment, ${ }^{224} \mathrm{~Pa}$ was produced via the ${ }^{209} \mathrm{Bi}\left({ }^{18} \mathrm{O}, 3 n\right){ }^{224} \mathrm{~Pa}$ reaction which has been previously reported [Fukuda 1986], but without a production cross section measurement. The Lawrence Berkeley National Laboratory 88-Inch Cyclotron provided a 20 electrical-nA beam of $111 \mathrm{MeV}$ (laboratory frame) ${ }^{18} \mathrm{O}^{5+}$. The beam energy was chosen such that the protactinium products that were able to recoil out of the target had approximately the same bombarding energy of $88 \mathrm{MeV}$ as used by Fukuda et al. This target is thick compared to the recoil range of the reaction products 
which recoil only out of approximately the last half of the target at the bombarding energy used.

\subsubsection{The ${ }^{249} \mathrm{Bk}\left({ }^{18} \mathrm{O}, \mathrm{x} n\right)^{267-\mathrm{x}} \mathrm{Ha}$ reaction}

The hahnium was produced via the ${ }^{249} \mathrm{Bk}\left({ }^{18} \mathrm{O}, \mathrm{x} n\right){ }^{267-\mathrm{x}} \mathrm{Ha}$ reaction. The Lawrence Berkeley National Laboratory 88-Inch Cyclotron provided an intense beam of ${ }^{18} \mathrm{O}^{5+}$

\subsubsection{The ${ }^{249} \mathrm{Bk}\left({ }^{22} \mathrm{Ne}, 4,5 n\right){ }^{267,266} \mathrm{Bh}$ reaction}

The isotopes, ${ }^{267} \mathrm{Bh}$ and ${ }^{266} \mathrm{Bh}$, were produced in bombardments of a ${ }^{249} \mathrm{Bk}$ target with ${ }^{22} \mathrm{Ne}$ ions at the LBNL 88-Inch Cyclotron. Two bombarding energies were used: 148 and $153 \mathrm{MeV}$ from the cyclotron (lab reference). This resulted in an energy in the berkelium target material of $117-\mathrm{MeV}$ and $123-\mathrm{MeV}$, respectively. These energies were chosen based on predictions made with the SPIT code (see section 5.1 for more details).

\subsection{Collection and Transport}

Products of the nuclear reactions recoiled out of the target and into a recoil chamber, located directly behind the target, that is continuously swept with He gas containing $\mathrm{KCl}$ aerosols to collect the recoiling reaction products. These products are then transported through a Teflon capillary (1.4-mm inside-diameter, 7-m long) to the Merry-Go-round (MG) rotating wheel in about a second [Wilk 1998], including retention time in the recoil chamber, with a transport efficiency of around $50 \%$. 


\subsection{Detection}

The MG detection system consists of a 20-inch-diameter fiberglass wheel, which is rotated at preset intervals between six pairs of passivated, ion-implanted planar silicon (PIPS) detectors. This provides simultaneous on-line collection and measurement of transported reaction products. A parentdaughter stepping

mode was used to provide detection of Figure 3.2 Parent-Daughter Mode Schematic

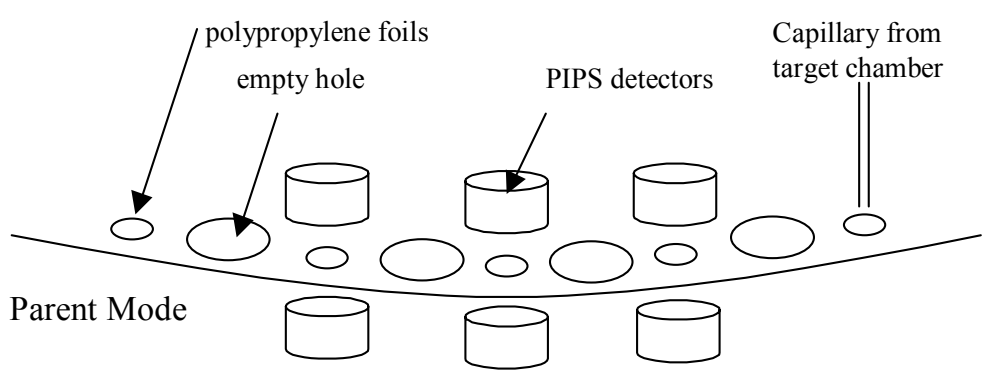
$\alpha-\alpha$ correlations with a greatly reduced background. The

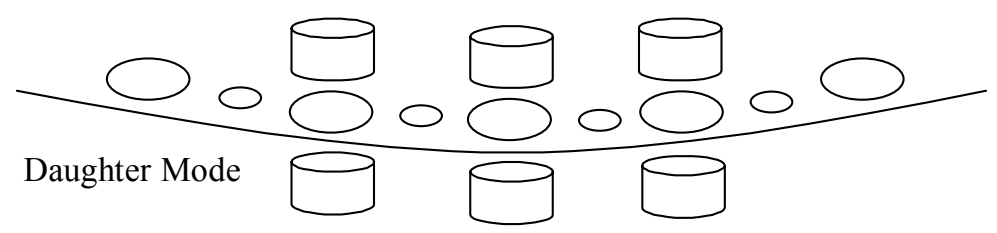
reaction products are deposited on 40 polypropylene films $\left(\sim 40 \mu \mathrm{g} / \mathrm{cm}^{2}\right)$ placed in every other position around the periphery of the 80 -position rotating collection wheel. During parentsearch mode, the wheel is double-stepped between the six pairs of $\alpha$-particle detectors until a possible parent decay is detected in a bottom detector. If an $\alpha$-particle is detected in the bottom detector within an energy window that is expected for the parent nucleus, it is assumed that the daughter nucleus has recoiled out of the collected sample and into the top detector. The probability of the daughter nucleus recoiling into the top detector was previously measured at about $65 \%$. When a possible parent decay 
event is detected, a daughter-search mode is initiated by single-stepping the wheel to move an empty position between the detectors in order to detect the daughter or granddaughter $\alpha$-decay in the absence of the activity on the collection foil. At the end of the daughter mode interval, the wheel is single-stepped again and parent-search mode is resumed.

\subsection{SISAK}

\subsubsection{Introduction to SISAK}

The SISAK 3 (Short-lived Isotope Separation by the AKufe technique) system coupled with LISSY (LIquid Scintillation SYstem) was developed to rapidly separate elements chemically and identify them. [Wierczinski 1994]. The system has been successfully used to extract group five and seven homologues [Wierczinski 1995] and to positively identify ${ }^{161} \mathrm{Hf}\left(\mathrm{T}_{1 / 2}=18 \mathrm{~s}\right)$ by $\alpha$-liquid-scintillation $(\alpha$-LS) spectroscopy [Wierczinski 1996b]. It also has been used successfully for the study of ${ }^{110} \mathrm{Ru}$ by gamma spectroscopy after the rapid extraction of ${ }^{110} \mathrm{Tc}$ [Altzitzoglou 1990]. The purpose of the following experiment was a first attempt to chemically separate and identify hahnium using SISAK, as well as to perform an operational test using protactinium produced on-line.

\subsubsection{SISAK 3}

SISAK 3 is an on-line, rapid, liquid-liquid extraction system. This system is in its third major incarnation, with the third system mainly differing from its predecessors by its much smaller centrifuges - it is a micro-SISAK. By on-line, it is 
meant that the system operates in a continuous fashion with an uninterrupted, continuously flowing stream throughout the system. This is in contrast to a batch-type setup in which a discontinuous series of batch type extractions are performed, either automated or by hand. Both techniques have strengths and drawbacks. The major strength of the SISAK style on-line system is its ability to chemically separate and detect nuclides with half-lives as short as a few seconds. At the heart of SISAK 3 is a series of specially-designed, very small-volume centrifuges (see Figure 3.3). The centrifuges have a volume of $0.3 \mathrm{ml}$ and can function with a flow rate up to $2 \mathrm{ml} / \mathrm{s}$ and down to less than $1 \mathrm{ml} / \mathrm{s}$. This equates to a minimum transportation time through SISAK 3 of about one second.

The first step in SISAK 3 after the recoil chamber and the aerosol transfer to SISAK 3 (described previously), is the degasser. This is one of the SISAK 3 centrifuges used to remove the helium transfer gas and dissolve the aerosols in the liquid phase. When this experiment took place in April 1996, it was thought that the aerosol must be dissolved in the liquid phase to get proper transfer; however, recently during a similar experiment in December 2000 at LBNL it was found that it is possible to send the aerosol directly into the organic phase. This is contrary to expectation, as the $\mathrm{KCl}$ aerosol is

Figure 3.3 SISAK Centrifuge.

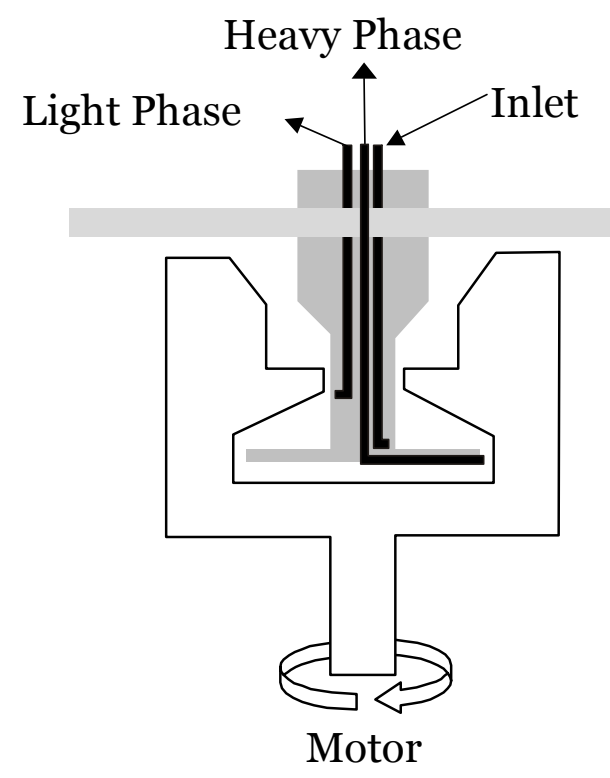


insoluble in the organic medium. However, the activity was successfully transferred to the organic phase. Perhaps the solvent washes the activity off the $\mathrm{KCl}$ aerosol clusters, or extracts it as a chloro-complex.

The next step of the SISAK system is the organic-aqueous extraction. This can be accomplished with either one or two centrifuges operating in many different configurations. It is possible to use the second extraction centrifuge to re-extract from a liquid phase, or to use it as an organic phase washing step. The scintillator cocktail, however, must be in the organic phase or be added to the organic phase. Thus the activity of interest must eventually follow the organic phase out of the SISAK system into the $\alpha$-LS system. For an example of an updated SISAK configuration as it was used in 2000, see Figure 4.5 and Photograph D.11.

\subsubsection{LISSY}

The LISSY is a flowing $\alpha$-LS spectroscopy system developed specifically for use with SISAK 3 [Wierczinski 1996b]. This system can perform time-correlated $\alpha$ pulse height analysis of a flowing organic solution. Under typical experimental conditions, it is capable of $\sim 400 \mathrm{keV}$ resolution with a detection efficiency of near $100 \%$. Electronic pulse-shape analysis reduces the beta background by on-line discrimination between $\alpha$ and $\beta$ events. 
During the April 1996 experiments the $\alpha$-LS system consisted of three cells.

Each $\alpha$-LS cell is a photomultiplier tube with a Teflon block facing it with a

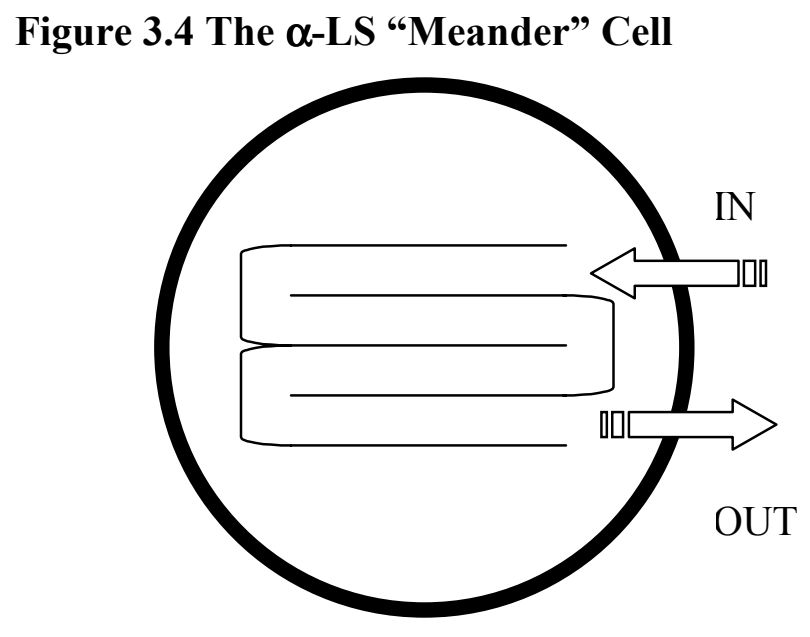

"meandering" path through it (see Figure 3.4 and Photograph D.10). The organic solution containing the scintillator cocktail meanders its way through the channel in the Teflon block. Any light from the scintilator will be detected with nearly perfect efficiency by the photomultiplier tube that is facing the solution. The cell has a volume of $4.8 \mathrm{ml}$. The three $\alpha$-LS cells were connected in series so that the solution after exiting one enters the next one immediately. The transport time through the cells is about a second each, but is totally dependent on the flow rate. By using three independent cells in series in this way, it is possible to determine the absolute time of each event based on the flow rate, and determine the half-life of short-lived isotopes.

\subsection{Data Acquisition}

The data were displayed with a Digital Equipment VAX workstation running the 
VMS operating system (see Photograph D.4). Previous to 1997, data were acquired via a software package developed at LBNL called CHAOS [Rathbun 1991] which will not be described in detail. Its operation is similar in theory to the software package that took its place. After 1997, the CHAOS software package had to be abandoned as the laboratory ceased to support the VAX hardware and the VMS operating system. The GOOSY (GSI Online Offline SYstem) software package developed at GSI [Essel 1987] was chosen to replace CHAOS. The Berkeley Gas-Filled Separator (BGS) came on-line during this period, and it also needed a robust and flexible acquisition system. It seemed most efficient to use one software package for both the MG and the BGS. This software was chosen largely due to the familiarity and expertise with this software package of Dr. Victor Ninov, a separator expert from Gesellschaft für Schwerionenforschung (GSI), Darmstadt, Germany now working on the BGS project. While GOOSY is being used by research groups around the world, it still has such a small user base that it can be considered custom software with all the difficulties inherent in a non-commercial package. Unfortunately, there are not really any commercial packages available that provide the flexibility and functionality that GOOSY provides. The flexibility of GOOSY provides an improvement over the CHAOS acquisition system.

The interface between the Computer Aided Measurement And Control (CAMAC) crate and the workstation was performed by a crate controller also developed at GSI called a CAMAC to VSB Computer (CVC). The CVC is a microcomputer with a Mitsubishi $68030 \mathrm{CPU}$ with SCSI, Ethernet, VSB, and CAMAC interfaces. A CVC is also made in a VERSAmodule Eurocard (VME) crate 
configuration, and one is currently being used with the BGS project. As expected, the CVC interfaces very well with a VMS system running GOOSY. The CVC runs a realtime Unix operating system called Lynx (http://www.lynx.com). This controller allows data to be written directly to tape though a SCSI port, as well as to stream the data over Ethernet. Streaming data over the network allows many computers simultaneously to observe the data as it is acquired. However, in practice for these experiments, this capability is used only to send data to the VAX running GOOSY. It is also possible to read and write data to tape from the VAX. Since the CVC and the VAX running GOOSY use exactly the same data structure, it is very easy to move stored data between the two systems for off-line data analysis. 


\section{Chapter 4: Group Five Elements}

\subsection{Nuclear Properties}

4.1.1 Cross section for the $\left.{ }^{209} \mathrm{Bi}\left({ }^{18} \mathrm{O}, 3 n\right)\right)^{224} \mathrm{~Pa}$ reaction and half-life of ${ }^{224} \mathrm{~Pa}$

Two previously reported half-lives for ${ }^{224} \mathrm{~Pa}$ were known to us at the time of our experiment. However, the standard deviation of these two measurements, $600 \pm 50 \mathrm{~ms}$ by Tove [Tove 1958] from the ${ }^{232} \mathrm{Th}(p, 9 n)$ reaction and $950 \pm 150 \mathrm{~ms}$ measured by Autmann et. al. [Autmann 1970] from the ${ }^{205} \mathrm{Tl}\left({ }^{22} \mathrm{Ne}, 3 n\right)$ reaction were not in agreement within the quoted errors and as such, we decided to make a new

measurement. In our experiment, ${ }^{224} \mathrm{~Pa}$ was produced via the ${ }^{209} \mathrm{Bi}\left({ }^{18} \mathrm{O}, 3 n\right){ }^{224} \mathrm{~Pa}$ reaction which has been previously reported by Fukuda et. al. [Fukuda 1986], but they gave no production cross section or additional half-life measurement. Unbeknownst to us, another group was investigating the same isotope using the same ${ }^{209}{ }^{3}\left({ }^{18} \mathrm{O}, 3 n\right)$ reaction, and between the time our work was completed and the results finally published [Wilk 1997], they reported a half life of 790 $600 \mathrm{~ms}$ [Liang 1996]. However, our half-life measurement of $855 \pm 19 \mathrm{~ms}$ is more precise and is consistent with their value within the quoted errors. Although the same reaction was used, they did not report a cross section. 


\subsubsection{Procedure and Data Analysis}

The energy calibration was performed on-line using known $\alpha$-particle energies from the following nuclides: ${ }^{211} \mathrm{Bi},{ }^{214} \mathrm{Ra},{ }^{211} \mathrm{Po},{ }^{212} \mathrm{Ac},{ }^{216} \mathrm{Fr},{ }^{212 \mathrm{~m}} \mathrm{Po}$. The transport efficiency was calculated by comparing ${ }^{211}$ Po detected on a catcher foil to that detected on the MG. A molybdenum catcher foil is placed behind the target in order to collect all the reaction products that recoil out of the target [McFarland 1982]. The catcher foil and the samples collected on the MG wheel were both counted off-line after allowing all the ${ }^{211} \mathrm{Po} p n$ transfer product to decay away $\left(\mathrm{t}^{1} / 2=0.516 \mathrm{~s}\right)$ leaving only the ${ }^{211}$ Po that is produced from the electron-capture decay of the ${ }^{211}$ At $2 p$ transfer product. The ${ }^{211}$ Po on the wheel and the catcher foil were observed to decay with half-

Figure 4.1 The $\alpha$-particle spectrum of products of the ${ }^{209} \mathrm{Bi}$ and ${ }^{18} \mathrm{O}$ reaction collected over a 1.3-hour period.

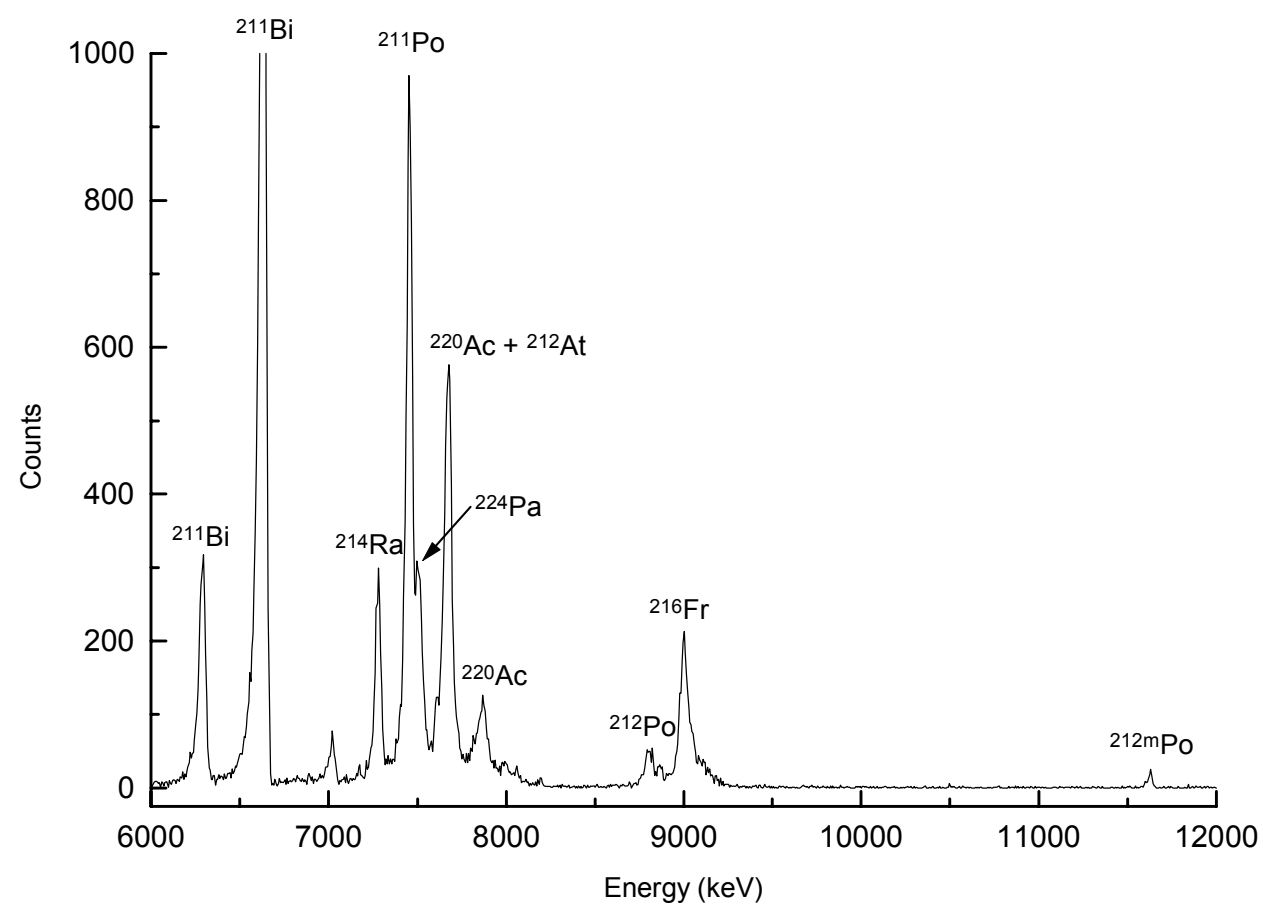


lives of $7.1 \pm 0.4 \mathrm{~h}$ and $7.18 \pm 0.03 \mathrm{~h}$, respectively, which agrees with the reported ${ }^{211} \mathrm{At}$ half-life of $7.214 \pm 0.007 \mathrm{~h}$ [Firestone 1996]. Another $2 n$ transfer product observed in the $\alpha$-energy spectrum was ${ }^{211} \mathrm{Bi}$ (see Figure 4.1 ), which was observed to decay with a $2.10 \pm 0.06 \mathrm{~min}$ half-life, in close agreement with the reported half-life of $2.14 \pm 0.02$ $\min$ [Firestone 1996].

The odd-odd nucleus ${ }^{224} \mathrm{~Pa}$ is not expected to have a significant beta-decay branch [Takahashi 1973] and appears to $\alpha$-decay mainly to a single nuclear level in ${ }^{220}$ Ac with an $\alpha$-particle energy of 7.49 MeV [Borggreen 1970, Firestone 1996]. Unfortunately, while $\alpha$-particles with this energy are identifiable in our $\alpha$-energy spectrum, they are largely obscured by the ${ }^{211}$ Po transfer product which decays via 7.45-MeV $\alpha$-particles (see Figure 4.1). To determine the production cross section and half-life of ${ }^{224} \mathrm{~Pa}, \alpha$-decays from its decay chain that were observed within $2 \mu \mathrm{s}$ in

Figure 4.2 Contour plot of $\alpha$-events occurring within $2 \mu \mathrm{s}$ in opposite detectors

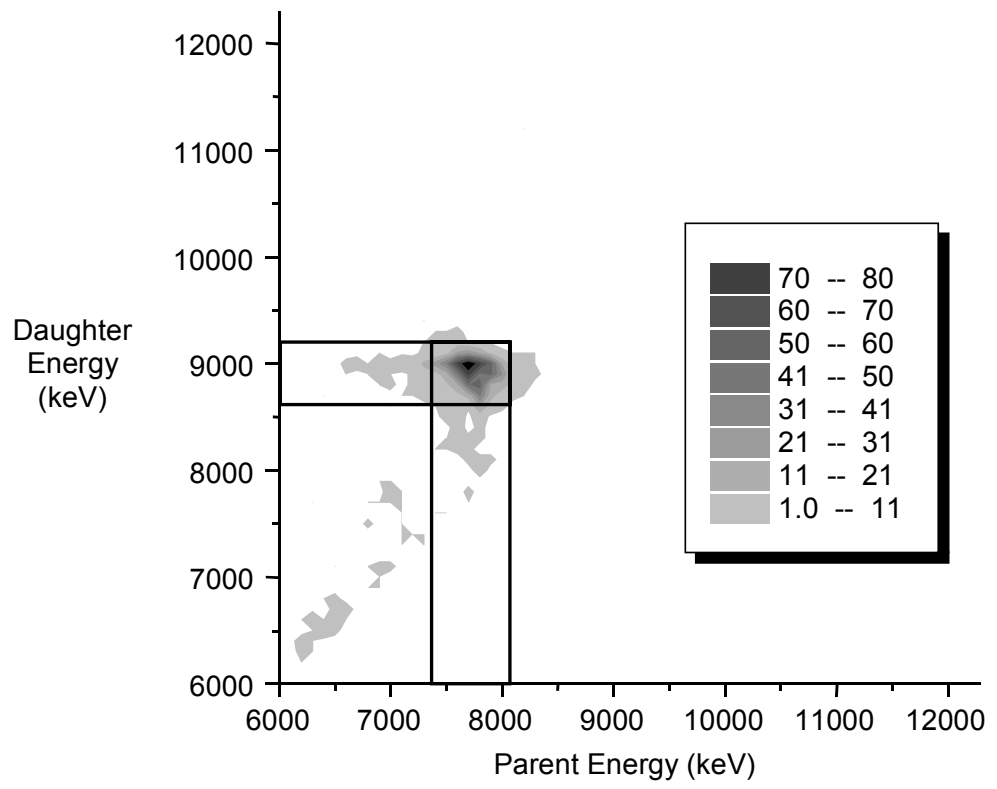


Figure 4.3 Alpha activity from the decay of ${ }^{220}$ Ac and ${ }^{216}$ Fr correlated within $2 \mu \mathrm{s}$.

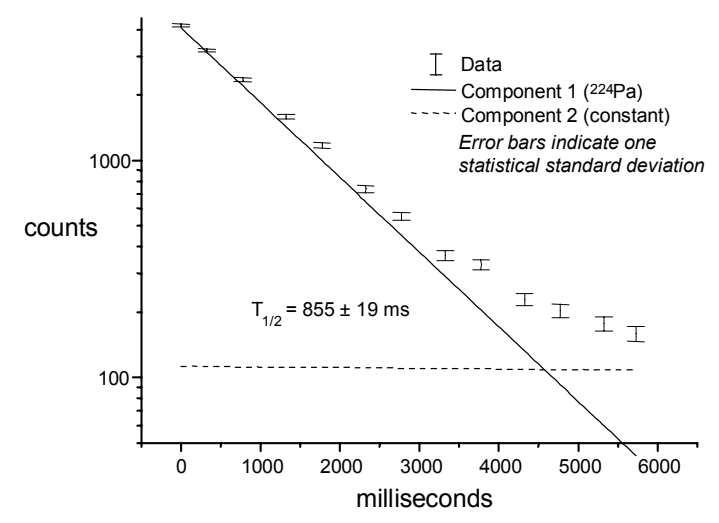

opposite detectors of a detector pair (see Figure 4.2) with $\alpha$ decay energies of ${ }^{220} \mathrm{Ac}\left(\mathrm{t}^{1} / 2=26\right.$ $\mathrm{ms} ; \alpha=7.610,7.680,7.790$, 7.850 MeV [Firestone 1996]) and ${ }^{216} \operatorname{Fr}\left(\mathrm{t}^{1 / 2}=0.7 \mu \mathrm{s} ; \alpha=9.01\right.$ MeV [Firestone 1996]) were used to identify ${ }^{224} \mathrm{~Pa}$.

By fitting the resultant decay curve with two components, a variable half-life and a fixed, very long half-life (both initial activities were allow to vary), the half-life of ${ }^{224} \mathrm{~Pa}$ was determined to be $855 \pm 19 \mathrm{~ms}$ (see Figure 4.3 ). The error limits in the halflife and the figure correspond to a confidence level of $68 \%$. Decay of anything with a half-life of much more than a minute would be too long to determine during the sixsecond detection interval. The long-lived component was due to ${ }^{211}$ Po correlated randomly with francium. Due to the short correlation time, we were unable to temporally discriminate between the two correlated events, which prevented us from analyzing for random correlations. From the data collected, we were also able to determine the production cross section to be $1.8 \pm 0.9 \mathrm{mb}$, by taking into account the detector geometric efficiency, capillary transport efficiency of $50 \pm 10 \%$, and capillary transport time of $1.0 \pm 0.3 \mathrm{~s}$. We calculated the cross section of this reaction to be 0.35 mb at this energy using the SPIT code [Wild 1988]. 


\subsubsection{Discussion}

Three other measurements of the half-life of ${ }^{224} \mathrm{~Pa}$ are currently known in addition to ours: $600 \pm 50 \mathrm{~ms}$ from the ${ }^{232} \mathrm{Th}(p, 9 n)$ reaction [Tove 1958 ], $950 \pm 150 \mathrm{~ms}$ from the ${ }^{205} \mathrm{Tl}\left({ }^{22} \mathrm{Ne}, 3 n\right)$ reaction [Autmann 1970] and $790 \pm 60 \mathrm{~ms}$ from the ${ }^{209} \mathrm{Bi}\left({ }^{18} \mathrm{O}\right.$, $3 n$ ) reaction [Liang 1996]. Tove's low value could be due to a misinterpretation of the very complex spectrum that was obtained. The disparity could lie in the misidentification of a ${ }^{223} \mathrm{Th}\left(\mathrm{t}^{1} / 2=0.65 \mathrm{~s}\right)$ decay peak produced by the ${ }^{232} \mathrm{Th}\left(p, p^{\prime} 9 n\right)$ reaction. Our value of $855 \pm 19 \mathrm{~ms}$ agrees with the other reported values, but is more precise.

\subsection{Chemical Properties}

\subsubsection{Introduction}

Hahnium has been shown to behave generally as a group five element. Previous experiments show that hahnium is sorbed on glass surfaces similarly to the group five elements niobium and tantalum, but unexpectedly remains in the aqueous phase with niobium when extracted into methylisobutylketone, unlike tantalum [Gregorich 1988]. Previous experiments have also shown that hahnium oxyhalides extract from chromatographic columns in a fashion analogous to protactinium. The actinides in the first half of the series are somewhat dissimilar to those of the trivalent second half. In chemical environments they often behave more like their associated main group elements. It seems more appropriate to place the lanthanides and actinides in the periodic table in a manner to reflect this. One will notice in Figure 1.2 that 
protactinium is placed under hahnium and the other group five elements.

In this manner, protactinium is described as a group five pseudo-homologue. The most stable oxidation state in aqueous solution is $\mathrm{Pa}(\mathrm{V})$. It has a tendency to form polymers. It is never seen as an uncoordinated cation due to its strong tendency to undergo hydrolysis. And most significantly for us, it has a nasty tendency to be adsorbed on most any available surface. These are all characteristics that protactinium shares with its group five homologues.

\subsubsection{SISAK 3 experiments with ${ }^{263,262} \mathrm{Ha}$ at LBNL}

The experiments at LBNL during April 1996 tested the SISAK system in a few different configurations. The general scheme in several permutations worked on during the experiment was a two-step extraction. The first step consisted of an organic phase of 1M Cyanex 471X (tri-isobutyl phosphorous sulfide) in toluene and an aqueous phase of $1 \mathrm{M} \alpha-\mathrm{HIB}$ or $1 \mathrm{M}$ lactic acid. The aqueous phase was extracted to second centrifuge. The aqueous phase was mixed with a new organic phase which consisted of $80 \mathrm{~g} / 1$ naphthalene, $1 \mathrm{~g} / 1$ dimethyl POPOP, $2 \%$ volume trioctyl amine (TOA), 2.5\% volume dodecanol, all thoroughly mixed in toluene. The organic phase was then extracted to the first cell of the $\alpha$-LS system.

In the first step, the Cyanex extraction removed the polonium from the aqueous phase. Polonium is a transfer product made in large amounts from lead impurities in the target. It is essential to remove this $\alpha$-decaying contamination to reduce the $\alpha$ background. The $\alpha$-HIB or lactic acid in the aqueous solution complexes the desired group five product for extraction with TOA in the second step. 
The second step is the main extraction step. The TOA selectively extracts the hahnium. The dimethyl POPOP is the scintillator and the naphthalene is an energy transfer molecule. The dodecanol is not absolutely necessary, but enhances the phase separation and therefore the efficiency of the system.

\subsubsection{SISAK 3 experiments with ${ }^{224} \mathrm{~Pa}$ at LBNL}

As mentioned previously in section 2.2.4 (page 15), protactinium acts as a group five psuedohomologue. Its extraction properties are similar to niobium and tantalum, and it can be extracted in high yield with trioctylamine from either lactic acid or $\alpha$-hydroxyisobutyric acid [Wierczinski 1995]. Since the ${ }^{224} \mathrm{~Pa}$ production cross section is much larger than that of any hahnium isotope, and its half-life and decay properties were ideal for testing the system for a hahnium experiment, it was chosen for on-line measurements with SISAK.

For this experiment the following general procedure and solutions were used, which are basically the same as those used for hahnium described in the previous section. The first step after degassing was an extraction with an organic phase of $1 \mathrm{M}$ Cyanex 471X (tri-isobutylphosphine sulfide) in toluene and an aqueous phase of $1 \mathrm{M}$ lactic acid. The aqueous phase was transferred to the next centrifuge where the aqueous phase was mixed with a new organic phase. This organic phase was $80 \mathrm{~g} / 1$ naphthalene, $1 \mathrm{~g} / 1$ dimethyl POPOP, $2 \%$ volume TOA, 2.5\% volume dodecanol, all thoroughly mixed in toluene. The organic phase was then extracted to the $\alpha$-LS system. The flowrate through the system was about $1 \mathrm{ml} / \mathrm{s}$. The energy resolution for the $\alpha$-LS system during this experiment was about $250 \mathrm{keV}$ [Wierczinski 2001]. 
The energy calibration was performed on-line using known $\alpha$-particle energies from the decay of ${ }^{227} \mathrm{Ac}$. The radon from a ${ }^{227} \mathrm{Ac}\left(\mathrm{T}_{1 / 2}=21.8 \mathrm{y}\right)$ source was added to the organic solution using another degasser whenever desired. The daughters ${ }^{219} \mathrm{Rn}$ $\left(\mathrm{E}_{\alpha}=6.819 \mathrm{MeV}, \mathrm{T}_{1 / 2}=3.96 \mathrm{~s}\right)$ and ${ }^{215} \mathrm{Po}\left(\mathrm{E}_{\alpha}=7.386 \mathrm{MeV}, \mathrm{T}_{1 / 2}=1.78 \mathrm{~ms}\right)$ were used for calibration. The transport efficiency was calculated during a later experiment described previously in section 4.1.1 .

\subsubsection{Results and Discussion}

During the continuous 90-hour experiment we expected to see 180 hahnium $\alpha$ decays, instead we saw less than ten. To test the system we used ${ }^{224} \mathrm{~Pa}$ produced by the ${ }^{209} \mathrm{Bi}\left({ }^{16} \mathrm{O}, n\right){ }^{224} \mathrm{~Pa}$ reaction. The SISAK $\alpha$-LS detected 650 protactinium $\alpha$-decays during a 40-minute bombardment. Based on the beam current of the cyclotron during the experiment, $50 \mathrm{nA}$, we calculated we should have seen about $2.6 \times 10^{4}{ }^{224} \mathrm{~Pa}$ decays during the 40 -minute bombardment. This is a chemical yield of only $2.5 \%$. The transit time through the system was found to be between 3.5 and 4.0 seconds. It was found during this experiment that recycling the organic phase works well, which is a technique that has been used recently to reduce waste and cost of expensive materials with SISAK 3.

Since it is well known that group five elements have a very high affinity for surfaces, the possibility of sorption onto the apparatus was investigated. The SISAK 3 centrifuge surfaces can either be made out of palladium-passivated titanium and PEEK, or entirely out of PEEK. In an experiment described here, and experiments by others, it was found that protactinium sorbs very well on titanium surfaces, but not as 
well on PEEK surfaces.

To test this, I built a ${ }^{233} \mathrm{~Pa}$

$\left(\mathrm{T}_{1 / 2}=27 \mathrm{~d}, \beta\right.$-emitter, $\mathrm{E}_{\gamma}=312$

$\mathrm{keV}$ ) “cow” by sorbing ${ }^{237} \mathrm{~Np}$ on

an anion exchange column (see

Figure 4.4). The ${ }^{233} \mathrm{~Pa}$ was eluted

from the column with $\sim 25 \mu \mathrm{L}$ of
Figure 4.4 Protactinium generator

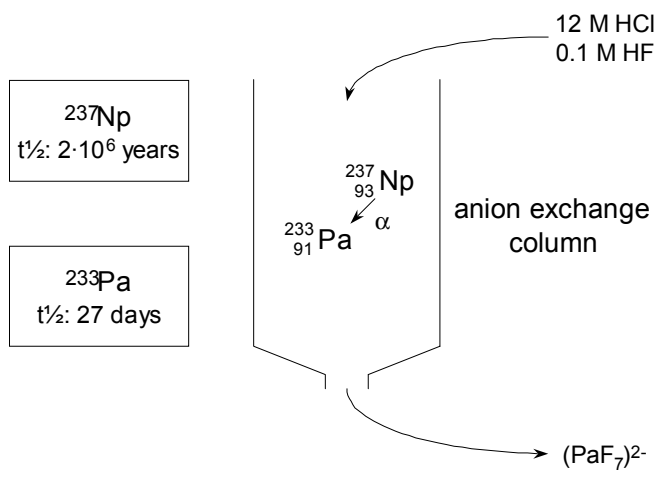

$12 \mathrm{M} \mathrm{HCl} / 0.1 \mathrm{M} \mathrm{HF}$ from and

deposited on various test material: Teflon, glass, and titanium foil. The solution was

gently evaporated under a warm heat lamp and the surface washed five times with 50

$\mu \mathrm{L}$ of $1 \mathrm{M} \alpha-\mathrm{HIB}$ to simulate the SISAK aqueous phase. The material and the $250 \mu \mathrm{L}$ of wash solution were then counted for $\gamma$ activity from ${ }^{233} \mathrm{~Pa}$ on a HPGe spectrometer. The percentage of protactinium left on the material was $1 \pm 1,4 \pm 1,18 \pm 2 \%$, for Teflon, glass, and titanium foil respectively [Wilk 1996].

For subsequent experiments, ${ }^{178} \mathrm{~W},{ }^{95} \mathrm{Nb}$ and ${ }^{233} \mathrm{~Pa}$ were run directly through SISAK 3 and similar results to my sorption experiments were obtained. Around $40 \%$ of the ${ }^{233} \mathrm{~Pa}$ activity was lost when using the palladium-passivated titanium centrifuges and about $5-10 \%$ of the ${ }^{233} \mathrm{~Pa}$ activity was lost when using the PEEK centrifuges. The centrifuges used during our experiment were constructed of PEEK, and not palladiumpassivated titanium. The tungsten and niobium activity that was lost when using either material was minimal [Wierczinski 2001, Alstad 1997].

It should be noted that it seems that protactinium ions and complexes may slowly change into a chemical form, perhaps colloidal, that can be removed by 
filtering but does not extract into TOA [Alstad 1997]. This tendency to form colloids is a common feature of the group five elements. However, this appears not to be a rapid process and should not affect on-line experiments. The presence of $0.05 \mathrm{M} \mathrm{HF}$ in the aqueous solution of lactic acid will solve the adsorption problem, and also seems to remove protactinium that has previously been sorbed onto experimental material [Alstadt 1997]. It is uncertain whether it is possible to effectively use HF during a SISAK 3 extraction at this time.

Why the protactinium and hahnium yield was so low during the experiments is a complex issue. It seems likely that some of the material was sorbed onto the apparatus itself. However, it does not seem possible based on subsequent experiments that this fully explains the large loss of activity. Other possibilities include that the degasser/mixer device did not function optimally, the phase separation and extraction from the centrifuges was poor, and/or that $\alpha$-decay events were obscured by the radiation background [Wierczinski 2001, Omtvedt 1998]. The first problem of getting the aerosol activity efficiently into solution has been addressed by redesigning the mixing device so that it increases solution contact with the aerosol [Wierczinski 2001]. Also, it has been shown that it is possible to transfer the activity directly to the organic phase [Omtvedt 2001]. The second problem was addressed by adding the scintillator cocktail after the extraction has taken place. This improved separation considerably but required the use of the rather expensive 1-methyl-naphthalene instead of naphthalene to reduce the volume of scintilator solution added [Omtvedt 1998]. In addition, another "boosting" centrifuge was added before the $\alpha$-LS system to reduce back-pressure on the last extraction centrifuge. To solve the third problem, it is now 
possible to use the BGS as a pre-separator which removes most interfering activities. Also, new electronic and computer aided filtering has been added to improve the discrimination of $\alpha$-particles from the beta and gamma background.

Figure 4.5 An example of a SISAK configuration [Omtvedt 2001]

Centrifuges: C1-degasser, $\mathrm{C}_{2} \& \mathrm{C}_{3}$-chemical separations, $\mathrm{C}_{4}$-booster

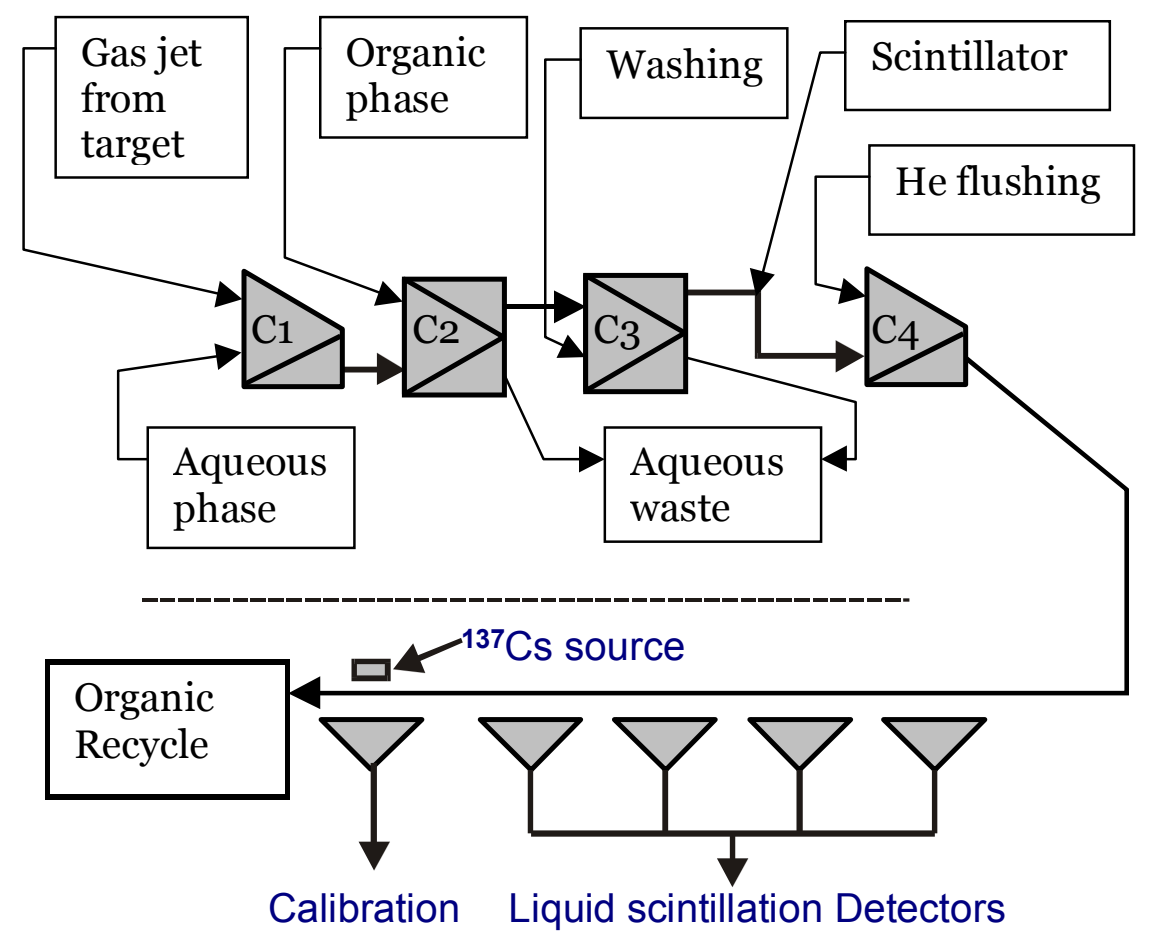

A recent series of experiments with rutherfordium at the 88-Inch Cyclotron during December 2000, tested the new improved SISAK system (see Figure 4.5 for an example configuration) that took all of these factors into account, and most importantly tested the BGS for use as a pre-separator. Also, a mother-daughter mode was added to the $\alpha$-LS system. In the case of an appropriate parent event, the specific scintillator cell was isolated and bypassed with the solvent flow stopped in the cell so that it is possible to wait for a long-lived daughter without having the entire 
experiment come to a stop (see Photograph D.11). A Recoil Transfer Chamber (RTC) was developed [Kirbach 2000] for the BGS that allows it to be used as a pre-separator for heavy element chemistry studies (see Photograph D.12). The RTC was connected to SISAK by a 18-meter long capillary. This interface was tested by bypassing the centrifuges of SISAK and taking the gas aerosol stream directly into the $\alpha$-LS system. The test was successful and the interface operated with a transport efficiency of around 30\%. The system was tested with $\mathrm{HDBP}$ extraction from $\mathrm{HNO}_{3}$ at a $\mathrm{HNO}_{3}$ concentration where both zirconium and hafnium extracted in high yield.

Rutherfordium did, indeed extract like a group four element and thus this was the first successful liquid-liquid extraction of a transactinide element. Four ${ }^{257} \mathrm{Rf}^{253} \mathrm{Lr}$ decay chains in a seven-hour run indicated that rutherfordium extracted together with zirconium and hafnium with high yield. The rutherfordium was observed by $\alpha-\alpha$ correlations both in the scintillation system and the focal plane detector of the BGS, verifying the identification. 


\section{Chapter 5: Group Seven Elements}

\subsection{Nuclear Properties of bohrium}

\subsubsection{Introduction}

Element 107, (bohrium, $\mathrm{Bh}$ ), was first identified as the isotope ${ }^{262} \mathrm{Bh}^{\mathrm{m}}$ produced in the ${ }^{209} \mathrm{Bi}\left({ }^{54} \mathrm{Cr}, n\right)$ reaction in 1981 by Münzenberg et al. [Münzenberg 1981] using the velocity filter Separator for Heavy Ion Production (SHIP) at the Gesellschaft für Schwerionenfourschung (GSI), Darmstadt, Germany. Oganessian et al. previously performed experiments in 1976 [Oganessian 1976a, 1976b, 1983] that were initially claimed to be the first synthesis of element 107 via the ${ }^{54} \mathrm{Cr}\left({ }^{209} \mathrm{Bi}, 2 n\right)$ reaction, based on observation of the spontaneous fission activity from the decay of ${ }^{261} \mathrm{Bh}$. In subsequent experiments, ${ }^{261} \mathrm{Bh}$ has been unambiguously identified by correlation with its $\alpha$-decay daughter ${ }^{259} \mathrm{Ha}$. Spontaneous fission activity was not detected and a low upper limit on the spontaneous fission branch was set [Münzenberg 1989]. To date, no spontaneous fission activity has been observed that can be attributed to spontaneous fission of any bohrium isotopes. Thus Oganessian et al. could not have been the first to observe the decay of the new element bohrium.

The properties of the isotopes of bohrium known previous to this work are:

${ }^{261} \mathrm{Bh}\left(\mathrm{T}_{1 / 2}=11.8 \mathrm{~ms} ; \mathrm{E}_{\alpha}=10.40,10.10,10.03 \mathrm{MeV}\right.$ [Münzenberg 1989]), ${ }^{262} \mathrm{Bh}$ $\left(\mathrm{T}_{1 / 2}=102 \mathrm{~ms} ; \mathrm{E}_{\alpha}=10.06,9.91,9.74 \mathrm{MeV}\right.$ [Münzenberg 1989]), ${ }^{262} \mathrm{Bh}^{\mathrm{m}}\left(\mathrm{T}_{1 / 2}=8 \mathrm{~ms} ; \mathrm{E}_{\alpha}=\right.$ 10.37, 10.24 [Münzenberg 1989]), and ${ }^{264} \mathrm{Bh}\left(\mathrm{T}_{1 / 2}=440 \mathrm{~ms} ; \mathrm{E}_{\alpha}=9.62,9.48 \mathrm{MeV}\right.$ [Hofmann 1995]). 
The experiments described in section 5.1.2 were undertaken to try to produce and identify the new neutron-rich isotopes of bohrium, ${ }^{266} \mathrm{Bh}$ and ${ }^{267} \mathrm{Bh}$. These isotopes were predicted to have significantly longer half-lives than previously known bohrium isotopes [Hatsukawa 1990, Smolańczuk 1995], possibly long enough to enable the first studies of bohrium chemical properties in subsequent experiments. Previous attempts to identify these isotopes by utilizing chromatographic separation have failed [Zvára 1984, Schädel 1995].

Zvára et al. bombarded ${ }^{249} \mathrm{Bk}$ with ${ }^{22} \mathrm{Ne}$ and looked for spontaneous fission by embedding quartz track detectors in the wall of chromathermatographic columns. Unfortunately, they were unable to locate any SF activity attributable to bohrium, but they were able to put an upper limit of $100 \mathrm{pb}$ on the SF partial cross section of any bohrium isotopes produced, assuming a half-life between $2 \mathrm{~s}$ and $2 \times 10^{4} \mathrm{~s}$. They bombarded their target with a flux of about $10^{17}$ particles per second with a beam energy of 116 - 119 in target MeV over the course of a 24 hour experiment [Zvára 1984].

Schädel et al. tried a chemical separation using a rather exotic target of ${ }^{254} \mathrm{Es}$ in 1994 [Schädel 1995a]. They bombarded this $50 \mu \mathrm{g} / \mathrm{cm}^{2}$ target with $113-$ and $116-\mathrm{MeV}$ ${ }^{16} \mathrm{O}^{5+}$ beams here at the 88 -Inch Cyclotron. The energies in the target were 93 and 96 $\mathrm{MeV}$, respectively, with a total dose of $2.54 \times 10^{16}$ and $1.90 \times 10^{16}$ particles. They chose their energies based on the maxima calculated from the SPIT [WILD 1988] and HIVAP [Reisdorf 1992] codes. They did not observe any bohrium decays, and calculated an upper-limit of a few nanobarns on the cross section for the bohrium isotopes produced via the ${ }^{254} \mathrm{Es}\left({ }^{16} \mathrm{O}, \mathrm{x} n\right){ }^{270-\mathrm{x}} \mathrm{Bh}$ reactions, assuming a half-life of $2 \mathrm{~s}$ or 
longer. If there were no $\mathrm{N}=162$ shell stabilization effects, they expected a cross section for the $4 n$ product of about $250 \mathrm{pb}$ based on experimental extrapolation, or $100 \mathrm{pb}$ based on HIVAP. They expected an enhancement of about an order of magnitude for every $\mathrm{MeV}$ increase in shell stability. Thus, they concluded that there was no evidence for a very significant shell stabilization. [Schädel 1995a]

Based on predicted Q-values for electron capture and $\alpha$-decay [Smolańczuk 1995], ${ }^{266} \mathrm{Bh}$ and ${ }^{267} \mathrm{Bh}$ should decay predominantly by $\alpha$-emission and possibly by spontaneous fission (SF). The $\alpha$-particle energies and half-lives for these isotopes are expected to be in the range of 8.7-9.3 MeV [Smolańczuk 1990] and 1-20 seconds [Hatsukawa 1990]. The previously reported decay characteristics of their Ha and Lr daughter nuclei, summarized in Figure 5.1, are: ${ }^{262} \mathrm{Ha}\left(\mathrm{T}_{1 / 2}=34 \mathrm{~s} ; \mathrm{E}_{\alpha}=8.45,8.53,8.67\right.$ $\mathrm{MeV}$ [Ghiorso 1971]), ${ }^{263} \mathrm{Ha}\left(\mathrm{T}_{1 / 2}=27 \mathrm{~s} ; \mathrm{E}_{\alpha}=8.35 \mathrm{MeV}[\operatorname{Kratz} 1992]\right),{ }^{258} \operatorname{Lr}\left(\mathrm{T}_{1 / 2}=3.9 \mathrm{~s}\right.$ [Gregorich 1992]; $\mathrm{E}_{\alpha}=8.60,8.62,8.57,8.65 \mathrm{MeV}$ [Ghiorso 1971]), and ${ }^{259} \mathrm{Lr}$ $\left(\mathrm{T}_{1 / 2}=6.34 \mathrm{~s} ; \mathrm{E}_{\alpha}=8.45 \mathrm{MeV}\right.$ [Gregorich 1992]). Kratz et al. [Kratz 1992], as listed in the Table of Isotopes [Firestone 1996], report an $\alpha$-particle energy of $8.35 \mathrm{MeV}$ for ${ }^{263} \mathrm{Ha}$, but other measurements [Gäggler 1994] indicate that ${ }^{263} \mathrm{Ha}$ might also decay by emission of 8.41-MeV $\alpha$-particles. 
5.1.2 Production of new isotopes of bohrium: ${ }^{266} \mathrm{Bh}$ and ${ }^{267} \mathrm{Bh}$

\subsubsection{Introduction}

New neutron rich isotopes,

Figure 5.1 Partial decay chain of ${ }^{266} \mathrm{Bh}$ and ${ }^{267} \mathrm{Bh}$. Decay properties of ${ }^{266} \mathrm{Bh}$ and ${ }^{267} \mathrm{Bh}$ in the dashed boxes are as measured during ${ }^{267} \mathrm{Bh}$ and ${ }^{266} \mathrm{Bh}$, were produced in these experiments.

bombardments of a ${ }^{249} \mathrm{Bk}$ target

with $117-\mathrm{MeV}$ and $123-\mathrm{MeV}^{22} \mathrm{Ne}$

ions at the LBNL 88-Inch

Cyclotron. Identification was made

by observation of correlated $\alpha$ -

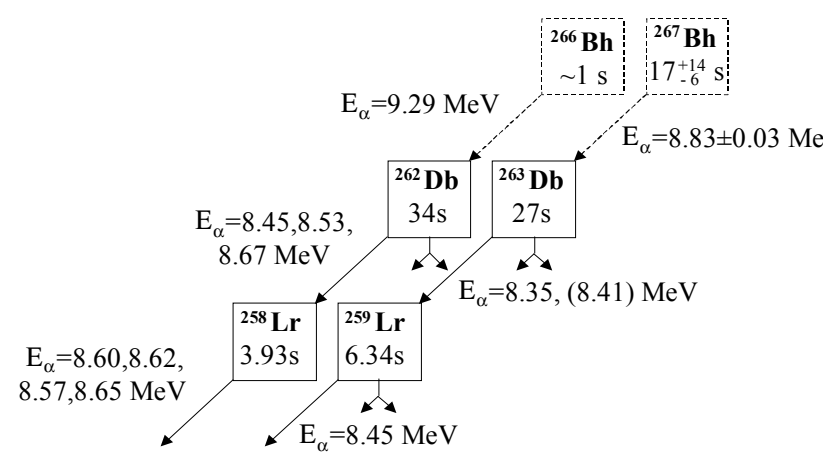

particle decays between the Bh

isotopes and their $\mathrm{Ha}$ and $\mathrm{Lr}$ daughters using the rotating wheel system described in

Section $3.5 .{ }^{267} \mathrm{Bh}$ was produced with a cross section of $\approx 70 \mathrm{pb}$ and decays with a

$17_{-6}^{+14} \mathrm{~s}$ half-life by emission of $\alpha$-particles with an average energy of $8.83 \pm 0.03 \mathrm{MeV}$.

One atom of ${ }^{266} \mathrm{Bh}$ was observed, decaying within one second by emission of a 9.29-

$\mathrm{MeV} \alpha$-particle.

\subsubsection{Procedure and Data Analysis}

The $\alpha$-particle energy resolution above the $\mathrm{MG}$ wheel was $\sim 40 \mathrm{keV}$, while the energy resolution of the detectors below was $\sim 100 \mathrm{keV}$ due to energy degradation in the polypropylene foils. The energy calibration was performed on-line using known $\alpha$ particle energies of ${ }^{212} \mathrm{Ra}\left(\mathrm{E}_{\alpha}=6.901 \mathrm{MeV}\right)$ and ${ }^{212} \mathrm{Po}^{\mathrm{m}}\left(\mathrm{E}_{\alpha}=11.650 \mathrm{MeV}\right)$. The transport efficiency was determined by comparing Md transfer product activities with 
those collected in a catcher-foil placed directly behind the target. A representative parent-mode $\alpha$-spectrum is shown in Figure 5.2. The total event rate seen by the detector array was about 5 events per second. The first detector pair saw $75 \%$ of the event rate, with the remaining $25 \%$ distributed equally among the other five detectors. The top and bottom detectors of an individual detector pair were exposed to approximately the same rate.

Figure 5.2 The $\alpha$-particle spectrum measured over the entire 62-hour experiment in detector pair three top, of products of the reaction of $117-\mathrm{MeV}^{22} \mathrm{Ne}$ with ${ }^{249} \mathrm{Bk}$.

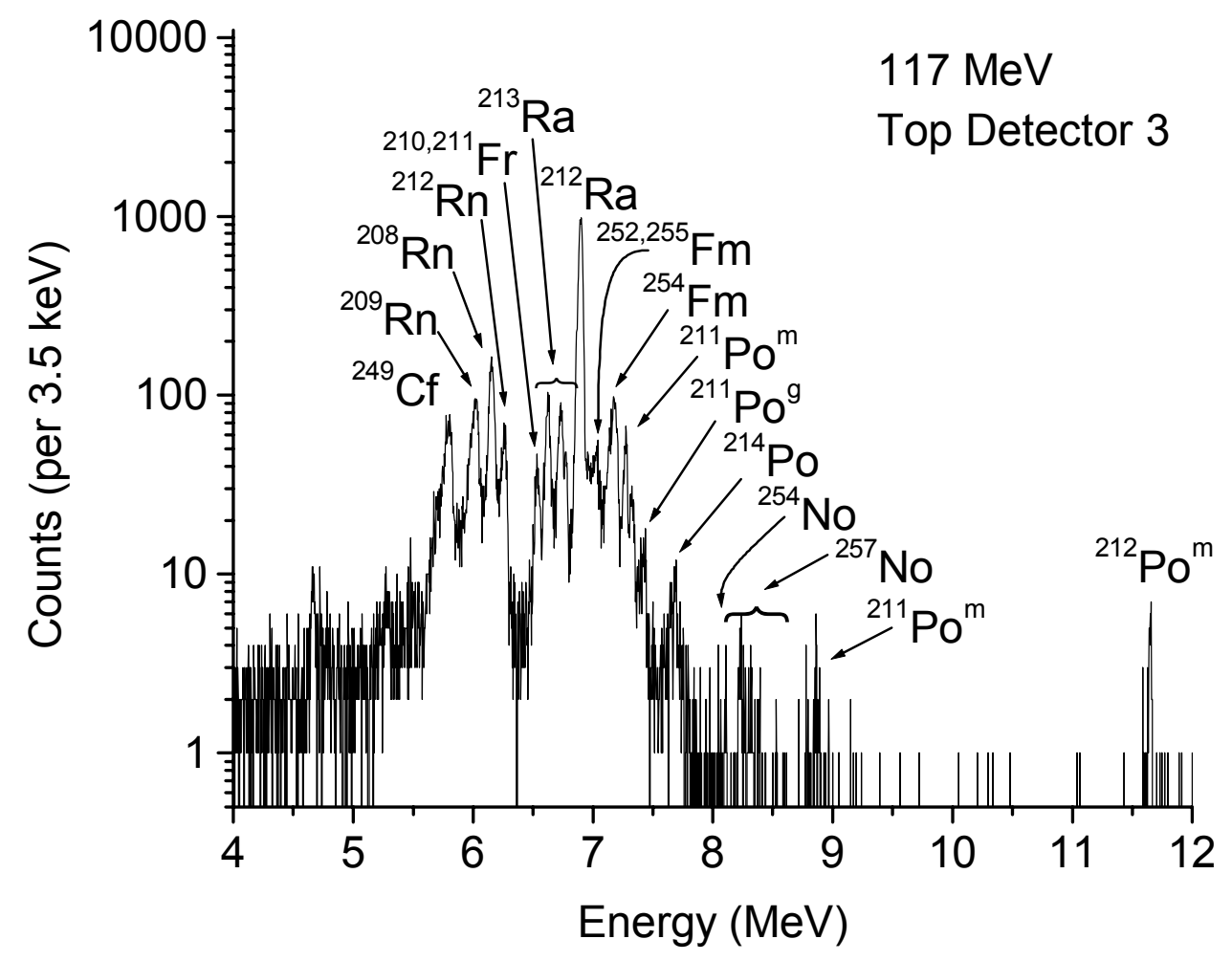

An off-line computer search was made for $\alpha$ - $\alpha$ correlations between Bh events $\left[8.6<\mathrm{E}_{\alpha}(\mathrm{MeV})<10.5\right]$ in parent-mode followed by daughter $\alpha$-events $\left[8.2<\mathrm{E}_{\alpha}(\mathrm{MeV})<8.7\right]$ detected in the same detector pair during the ensuing daughter 
mode search. Five atoms of ${ }^{267} \mathrm{Bh}, \mathrm{E}_{\alpha}$ ranging from 8.73 to $8.87 \mathrm{MeV}$, and one atom of ${ }^{266} \mathrm{Bh}$ with an $\mathrm{E}_{\alpha}$ of 9.29 $\mathrm{MeV}$, were identified during the experiment (See Table 5.1). Possible summing with conversion electrons from population of levels above the ground state and energy degradation in the polypropylene foil make it impossible to determine conclusively if more than one ${ }^{267} \mathrm{Bh} \alpha$-group is present. The average $\alpha$ energy is $8.83 \pm 0.03 \mathrm{MeV}$. The calculated ${ }^{267} \mathrm{Bh}$ half-life, fitted to the data using the maximum likelihood technique (MLDS) [Gregorich 1991] allowing the half-life and initial activity to vary with a single component fit, is $17_{-6}^{+14} \mathrm{~s}$. The five $\alpha$-events attributed to the $\alpha$ decay of ${ }^{267} \mathrm{Bh}$ daughter nuclei are consistent with ${ }^{263} \mathrm{Ha}$ and ${ }^{259} \mathrm{Lr}$. Assuming $\alpha$-decay is the dominant decay mode, the ${ }^{249} \mathrm{Bk}\left({ }^{22} \mathrm{Ne}, 4 n\right){ }^{267} \mathrm{Bh}$ cross section is $58_{-15}^{+33} \mathrm{pb}$ at 116 to $118 \mathrm{MeV}$ and is $96_{-25}^{+55} \mathrm{pb}$ at 122 to $124 \mathrm{MeV}$. Using the SPIT code [Wild 1988], we calculated that the maximum cross section for the ${ }^{249} \mathrm{Bk}\left({ }^{22} \mathrm{Ne}, 4 n\right){ }^{267} \mathrm{Bh}$ reaction is $\sim 32 \mathrm{pb}$ at $117 \mathrm{MeV}$ and $\sim 14 \mathrm{pb}$ at $123 \mathrm{MeV}$.

Of the 609 events during the experiment that initiated daughter mode, about half of them occurred in the first detector with the balance fairly evenly distributed among the remaining five detectors. About $13 \%$ of the time during the experiment was spent in daughter mode. We observed five cases during the entire experiment where $\alpha$ events in daughter mode with $\left[8.2<\mathrm{E}_{\alpha}(\mathrm{MeV})<8.7\right]$ were observed in detector pairs different from that in which the initiating parent event was observed. Assuming that these random daughter events are evenly distributed among the detectors, we estimate that approximately one of the five ${ }^{267} \mathrm{Bh} \alpha-\alpha$ correlations reported is due to a random correlation of unrelated $\alpha$-decays. Based on this random daughter rate, the expected 
number of random $\alpha-\alpha-\alpha$ triple correlations is 0.0016 .

Table 5.1 List of correlations between parent events $\left(8.6<\mathrm{E}_{\alpha}<10.5 \mathrm{MeV}\right)$ and daughter events $\left(8.2<\mathrm{E}_{\alpha}<8.7 \mathrm{MeV}\right)$. The initiating parent event, each subsequent $\alpha$-decay that occurred within the energy window, its isotopic assignment, alpha energy, and time are listed for each event.

\begin{tabular}{|c|c|c|c|c|c|c|c|c|c|}
\hline & Parent & $\alpha_{1}$ & Time $^{\mathrm{c}}$ & Isotope & $\alpha_{2}$ & Time $^{\mathrm{d}}$ & Isotope & $\overline{\alpha \alpha_{3}}$ & time $^{\mathrm{e}}$ \\
\hline $1^{\mathrm{a}}$ & ${ }^{267} \mathrm{Bh}$ & $8.83 \mathrm{MeV}$ & $5.26 \mathrm{~s}$ & ${ }^{263} \mathrm{Ha}$ or ${ }^{259} \mathrm{Lr}$ & $8.47 \mathrm{MeV}$ & $59.04 \mathrm{~s}$ & & & \\
\hline $2^{a}$ & ${ }^{267} \mathrm{Bh}$ & $8.87 \mathrm{MeV}$ & $24.67 \mathrm{~s}$ & ${ }^{263} \mathrm{Ha}$ & $8.39 \mathrm{MeV}$ & $35.02 \mathrm{~s}$ & & & \\
\hline $3^{\mathrm{a}}$ & ${ }^{267} \mathrm{Bh}$ & $8.87 \mathrm{MeV}$ & $45.15 \mathrm{~s}$ & ${ }^{263} \mathrm{Ha}$ & $8.39 \mathrm{MeV}$ & $24.49 \mathrm{~s}$ & & & \\
\hline $4^{b}$ & ${ }^{267} \mathrm{Bh}$ & $8.73 \mathrm{MeV}$ & $2.71 \mathrm{~s}$ & ${ }^{263} \mathrm{Ha}$ or ${ }^{259} \mathrm{Lr}$ & $8.46 \mathrm{MeV}$ & $51.90 \mathrm{~s}$ & & & \\
\hline $5^{b}$ & ${ }^{267} \mathrm{Bh}$ & $8.84 \mathrm{MeV}$ & $21.83 \mathrm{~s}$ & ${ }^{263} \mathrm{Ha}$ & $8.36 \mathrm{MeV}$ & $26.49 \mathrm{~s}$ & & & \\
\hline $6^{b}$ & ${ }^{266} \mathrm{Bh}$ & $9.29 \mathrm{MeV}$ & $0.87 \mathrm{~s}$ & ${ }^{262} \mathrm{Ha}$ & $8.54 \mathrm{MeV}$ & $27.83 \mathrm{~s}$ & ${ }^{258} \mathrm{Lr}$ & $8.74 \mathrm{MeV}$ & $0.04 \mathrm{~s}$ \\
\hline & $\mathrm{d}_{\mathrm{t}}^{\mathrm{a}}$ & $\begin{array}{l}\text { 16-118 Mel } \\
22-124 \mathrm{Me} \\
\text { me after en } \\
\text { me after } \alpha_{1} \\
\text { me after } \alpha_{2}\end{array}$ & $\begin{array}{l}{ }^{2} \mathrm{Ne} \\
{ }^{2} \mathrm{Ne} \\
\text { f } 10-\mathrm{s}\end{array}$ & ction & & & & & \\
\hline
\end{tabular}

During the entire experiment, there was only one instance where a potential parent event (at $9.29 \mathrm{MeV}$ ) was followed by two $\alpha$ particles with $\left[8.2<\mathrm{E}_{\alpha}(\mathrm{MeV})<8.7\right]$ in the daughter mode. The details of this event (number 6) are listed at the bottom of Table 5.1. The daughter-mode energies and lifetimes are consistent with those expected for ${ }^{262} \mathrm{Ha}$ and ${ }^{258} \mathrm{Lr}$. On this basis, we assign the 9.29-MeV event to the decay of ${ }^{266} \mathrm{Bh}$ produced in the ${ }^{249} \mathrm{Bk}\left({ }^{22} \mathrm{Ne}, 5 n\right)$ reaction. This triple correlation occurred during the higher energy bombardment, supporting assignment of the $5 n$-exit channel.

Since the wheel stepping time in this experiment was $10 \mathrm{~s}$ to optimize the search for isotopes in the 10-30 $\mathrm{s}$ range, the ${ }^{266} \mathrm{Bh}$ production cross section obtained from this measurement is strongly dependent on the assumed ${ }^{267} \mathrm{Bh}$ half-life. According to $\alpha$ decay systematics [Hatsukawa 1990] the unhindered half-life for 9.29- $\mathrm{MeV}^{266} \mathrm{Bh}$ decay should be $\sim 0.5 \mathrm{~s}$. An $\alpha$-hindrance factor between 2 and 20 would correspond to a half-life of 1-10 s. A half-life in this range would indicate a cross section between 
250 and $25 \mathrm{pb}$, taking into account the experimental conditions. The cross section calculated with SPIT was $3 \mathrm{pb}$ at 122 to $124 \mathrm{MeV}$.

\subsubsection{Further investigation of the nuclear properties of ${ }^{266} \mathrm{Bh}$}

In the previous experiment we determined the production cross section and decay properties of the longest lived isotope of element 107 (bohrium): ${ }^{267} \mathrm{Bh}$. This work on the physical properties of ${ }^{267} \mathrm{Bh}$ also gave us a brief glimpse at a single atom of ${ }^{266} \mathrm{Bh}$. Unfortunately, we were not able to determine the cross section or decay properties of ${ }^{266} \mathrm{Bh}$ in any detail due to limitations in the detection system that was optimized for longer-lived isotopes. Subsequently we performed another experiment to make an accurate measurement of the decay properties of ${ }^{266} \mathrm{Bh}$ and its cross section. During the fall of 1999 we worked with our collaborators at the Paul Scherrer Institute in Switzerland to perform the first study of the chemical properties of bohrium [Eichler 2000a,b,c]. Better data on ${ }^{266} \mathrm{Bh}$ were needed to optimize the experiments planned for Fall 2000 (see Section 5.2 ).

Since we have already performed a successful experiment on the neighboring isotope ${ }^{267} \mathrm{Bh}$, and observed one ${ }^{266} \mathrm{Bh}$ decay, we anticipated observing several more

${ }^{266} \mathrm{Bh}$ nuclei with some minor experimental modifications to our counting system. The apparatus for this experiment was identical to that used in the previous experiment, save for adjustment of counting intervals to 1.5 seconds in anticipation of a short-lived isotope, with a daughter interval of 2 minutes to detect the ${ }^{262} \mathrm{Ha}$ and ${ }^{258} \mathrm{Lr}$ daughters.

\subsubsection{Procedure and Data Analysis}

As mentioned above, the procedure followed for this experiment was identical to 
the experiment performed earlier, except for a different set of parent and daughter mode step times. The daughter-mode was initiated during any observation of an $\alpha-$ particle in a bottom detector between 8.8 and $10.5 \mathrm{MeV}$. This energy was chosen to exclude as much contaminating activity from polonium as possible. Approximately $4 \times 10^{18}$ ions were delivered to the target over the course of four days at an average cyclotron beam current of $2 \mathrm{e} \mu \mathrm{A}$. We expected to see a couple ${ }^{266} \mathrm{Bh}$ decay chains per day, assuming a one-second half-life. After an off-line study of the data, there were no correlations above background that were attributable to bohrium. There was one parent mode initiating event at $8.9 \mathrm{MeV}$ that occurred in the first bottom detector followed by another decay in the same detector $36 \mathrm{~s}$ later at $8.7 \mathrm{MeV}$. Unfortunately, there are other random $\alpha-\alpha$ correlations between unrelated detector pairs within the expected energy window that indicated with high probability that this could be also a random correlation.

Preliminary results from the Fall 2000 bohrium gas phase experiment also failed to find long-lived $\left(\mathrm{T}_{1 / 2}>5 \mathrm{~s}\right){ }^{266} \mathrm{Bh}$. A description of the gas-chemistry experiments is given in Section 5.2 . If the half-life of ${ }^{266} \mathrm{Bh}$ is much less than one second, we would not have observed it and it would not be useful for a chemical experiment.

\subsubsection{Discussion}

The new nuclides ${ }^{266} \mathrm{Bh}$ and ${ }^{267} \mathrm{Bh}$ have been observed to decay via $\alpha$ emission.

${ }^{267} \mathrm{Bh}$ has a half-life of $17_{-6}^{+14} \mathrm{~s}$, and emits $\alpha$-particles with an average energy of $8.83 \pm 0.03 \mathrm{MeV}$. One event with an $\alpha$-particle energy of 9.29 MeV and an estimated half-life of 1-10 s was attributed to ${ }^{266} \mathrm{Bh}$ based on the observed triple $\alpha$-correlation. 
We were not able to determine the fission decay properties of either isotope due to fission contamination that is attributed to ${ }^{256} \mathrm{Fm}$; about 12 fissions per hour were measured in coincidence in the top and bottom of each detector pair. The predicted and measured cross sections for the production of ${ }^{266,267} \mathrm{Bh}$ are summarized in Table 5.2. The measured ${ }^{266} \mathrm{Bh}$ cross section from the first bohrium experiment is a gross estimate based on one event and is highly dependent on the assumed half-life. Similar reactions have been used for the production of ${ }^{266} \mathrm{Sg}$ and ${ }^{265} \mathrm{Sg}$. These reactions are also presented together with the SPIT predictions and the experimentally measured production cross sections in Table 5.2.

Table 5.2 Experimental cross sections and SPIT predictions for the reactions ${ }^{22} \mathrm{Ne}$ on ${ }^{249} \mathrm{Bk}$ and ${ }^{22} \mathrm{Ne}$ on ${ }^{248} \mathrm{Cm}$.

\begin{tabular}{clccc}
\hline Reaction & \multicolumn{1}{c}{$\sigma_{\text {exp }}$} & Energy & $\sigma_{\text {SPIT }}$ & Reference \\
\hline${ }^{249} \mathrm{Bk}\left({ }^{22} \mathrm{Ne}, 4 n\right){ }^{267} \mathrm{Bh}$ & $\sigma_{\text {exp. }}=58_{-15}^{+33} \mathrm{pb}$ & $116-118 \mathrm{MeV}$ & $\sigma_{\text {SPIT }}=32 \mathrm{pb}$ & [Wilk 2000] \\
${ }^{249} \mathrm{Bk}\left({ }^{22} \mathrm{Ne}, 4 n\right){ }^{267} \mathrm{Bh}$ & $\sigma_{\text {exp. }}=96_{-25}^{+55} \mathrm{pb}$ & $122-124 \mathrm{MeV}$ & $\sigma_{\text {SPIT }}=14 \mathrm{pb}$ & [Wilk 2000] \\
${ }^{249} \mathrm{Bk}\left({ }^{22} \mathrm{Ne}, 5 n\right){ }^{266} \mathrm{Bh}$ & $\sigma_{\text {exp. }}=250-25 \mathrm{pb}$ & $122-124 \mathrm{MeV}$ & $\sigma_{\text {SPIT }}=3 \mathrm{pb}$ & [Wilk 2000] \\
${ }^{248} \mathrm{Cm}\left({ }^{22} \mathrm{Ne}, 4 n\right){ }^{266} \mathrm{Sg}$ & $\sigma_{\text {exp. }}=25 \mathrm{pb}$ & $120-124 \mathrm{MeV}$ & $\sigma_{\text {SPIT }}=70 \mathrm{pb}$ & [Türler 1998a] \\
${ }^{248} \mathrm{Cm}\left({ }^{22} \mathrm{Ne}, 5 n\right){ }^{265} \mathrm{Sg}$ & $\sigma_{\text {exp. }}=240 \mathrm{pb}$ & $120-124 \mathrm{MeV}$ & $\sigma_{\text {SPIT }}=15 \mathrm{pb}$ & [Türler 1998a] \\
\hline
\end{tabular}

These results show that SPIT overestimates the $4 n$ cross section by about a factor of 3 , but underestimates the $5 n$ reaction by a factor of about 16 . From these systematics, the experimental cross section of around $250-25 \mathrm{pb}$ for the ${ }^{249} \mathrm{Bk}\left({ }^{22} \mathrm{Ne}, 5 n\right)$ ${ }^{266} \mathrm{Bh}$ reaction at $122-124 \mathrm{MeV}$ is reasonable. As shown in Table 5.2, the measured cross sections of $25-250 \mathrm{pb}$ and $58_{-15}^{+33} / 96_{-25}^{+55} \mathrm{pb}$ for ${ }^{266} \mathrm{Bh}$ and ${ }^{267} \mathrm{Bh}$, respectively, are in agreement with the observed trends for $4 n$ and $5 n$ exit channels for analogous reactions [Kratz 1992, Lane 1996, Türler 1998, Lazarev 1994, Ghiorso 1970]. Our 
measured production cross section for ${ }^{267} \mathrm{Bh}$ is consistent with expectations based on calculations and the experimental systematics. The lifetime of the new isotope ${ }^{267} \mathrm{Bh}$ is sufficiently long for studies of the chemical properties of element 107 in either the aqueous or gas phase with fast separation techniques currently in use [Trautmann 1995,Wierczinski 1996a].

Table 5.3 Reactions analogous to the ${ }^{22} \mathrm{Ne}$ on ${ }^{249} \mathrm{Bk}$ reaction showing similar $4 n$ and $5 n$ exit channel systematics.

\begin{tabular}{c|c|c|c}
\hline \hline Reaction & $5 n$ & $4 n$ & References \\
\hline${ }^{249} \mathrm{Bk}\left({ }^{22} \mathrm{Ne}, \mathrm{x} n\right){ }^{267,266} \mathrm{Bh}$ & $25-250 \mathrm{pb}$ & $58_{-15}^{+33} \mathrm{pb}, 96_{-25}^{+55} \mathrm{pb}$ & [Wilk00] \\
${ }^{248} \mathrm{Cm}\left({ }^{22} \mathrm{Ne}, \mathrm{x} n\right){ }^{266,265} \mathrm{Sg}$ & $240 \mathrm{pb}$ & $80 \mathrm{pb}$ & [Türler 1998a]-[Lazarev 1994] \\
$\left.{ }^{249} \mathrm{Bk}\left({ }^{18} \mathrm{O}, \mathrm{x} n\right)\right)^{263,262} \mathrm{Ha}$ & $6 \mathrm{nb}$ & $2 \mathrm{nb}$ & [Kratz 1992] \\
$\left.{ }^{244} \mathrm{Pu}\left({ }^{22} \mathrm{Ne}, \mathrm{x} n\right)\right)^{262,261} \mathrm{Rf}$ & $3 \mathrm{nb}$ & $0.7 \mathrm{nb}$ & [Lane 1996]-[Ghiorso 1970] \\
\hline \hline
\end{tabular}

\subsection{Chemical properties of bohrium}

\subsubsection{On-line bohrium experiment at PSI}

The discovery of ${ }^{267} \mathrm{Bh}$ with a half-life of $\sim 17 \mathrm{~s}$ in the previously described experiment (section 5.1.2), provided an ideal candidate for gas phase chromatographic separation studies with the Swiss On-line Gas Chromatography Apparatus (OLGA) III, since the typical separation time with this device is on the order of $5 \mathrm{~s}$. The experiment is based on the assumption that Bh behaves as a group 7 element. These elements form very volatile oxychlorides in $\mathrm{HCl} / \mathrm{O}_{2}$ (gas).

Between August 20 and October 26, 1999, the first half of an experiment to study Bh chemistry was performed at the Paul Scherrer Institut (PSI), Villigen, Switzerland [Eichler 2000a,b,c]. A $670 \mu \mathrm{g} / \mathrm{cm}^{2}{ }^{249} \mathrm{Bk}$ target prepared at LBNL (section 
3.2.2) was irradiated with ${ }^{22} \mathrm{Ne}^{6+}$ at $118 \mathrm{MeV}$ for a total beam dose of $3.0 \times 10^{18}$ particles. This was the same target used previously at the 88-Inch Cyclotron in the discovery of the long lived bohrium isotopes. For this experiment, $100 \mu \mathrm{g} / \mathrm{cm}^{2}$ of ${ }^{159} \mathrm{~Tb}$ was deposited on its surface so that ${ }^{179} \mathrm{Re}$ would be also produced during bombardment, serving as a yield monitor for the chemical separation. The behavior of Bh was investigated at $180^{\circ}, 150^{\circ}$, and $75^{\circ} \mathrm{C}$ where Re still passes through the column with greater than $80 \%$ relative yield. The reaction products were transported by $\mathrm{He}$ $(1 \mathrm{~L} / \mathrm{m})$ loaded with carbon aerosol to the reaction oven of the gas chromatography system where they were oxidized at $1000^{\circ} \mathrm{C}$ by addition of $\mathrm{HCl}(50 \mathrm{~mL} / \mathrm{m})$ gas and $\mathrm{O}_{2}$ $(50 \mathrm{~mL} / \mathrm{min})$ gas to form the volatile chloride and oxychloride molecules. These molecules were then separated, depending on volatility and half-life, by isothermal gas adsorption chromatography ( $\sim 3 \mathrm{~s})$ on the interior of a quartz tube $(1.5 \mathrm{~m}$ length, $1.5 \mathrm{~mm}$ inside diameter). In a reclustering chamber, the transported volatile species were then reattached to $\mathrm{CsCl}$ particles in $\mathrm{He}(1.1 \mathrm{l} / \mathrm{min})$ and transported to the ROMA (ROtating wheel Multidetector Analyser) counting system. The ROMA system is very similar to our MG system, described previously in section 3.5. The thickness of the polypropylene films that were placed around the periphery of the ROMA wheel was 30 to $40 \mu \mathrm{g} / \mathrm{cm}^{2}$. The ROMA system differs from the MG in that it uses a vertically mounted, rather than a horizontal wheel, but otherwise they are functionally similar. In this experiment, the films were prepared manually by stretching grocery store variety plastic wrap. The majority of the films were prepared by Robert Eichler and he was able to prepare films that were as good and reproducible as our foils prepared in 
building 70A for my experiments with the "Magic Mushroom" heated, pneumatic plastic stretching device. For a diagram of the on-line isothermal gas chromatography device, OLGA III, see Figure 5.3.

Figure 5.3 OLGA III [Eichler 2001]

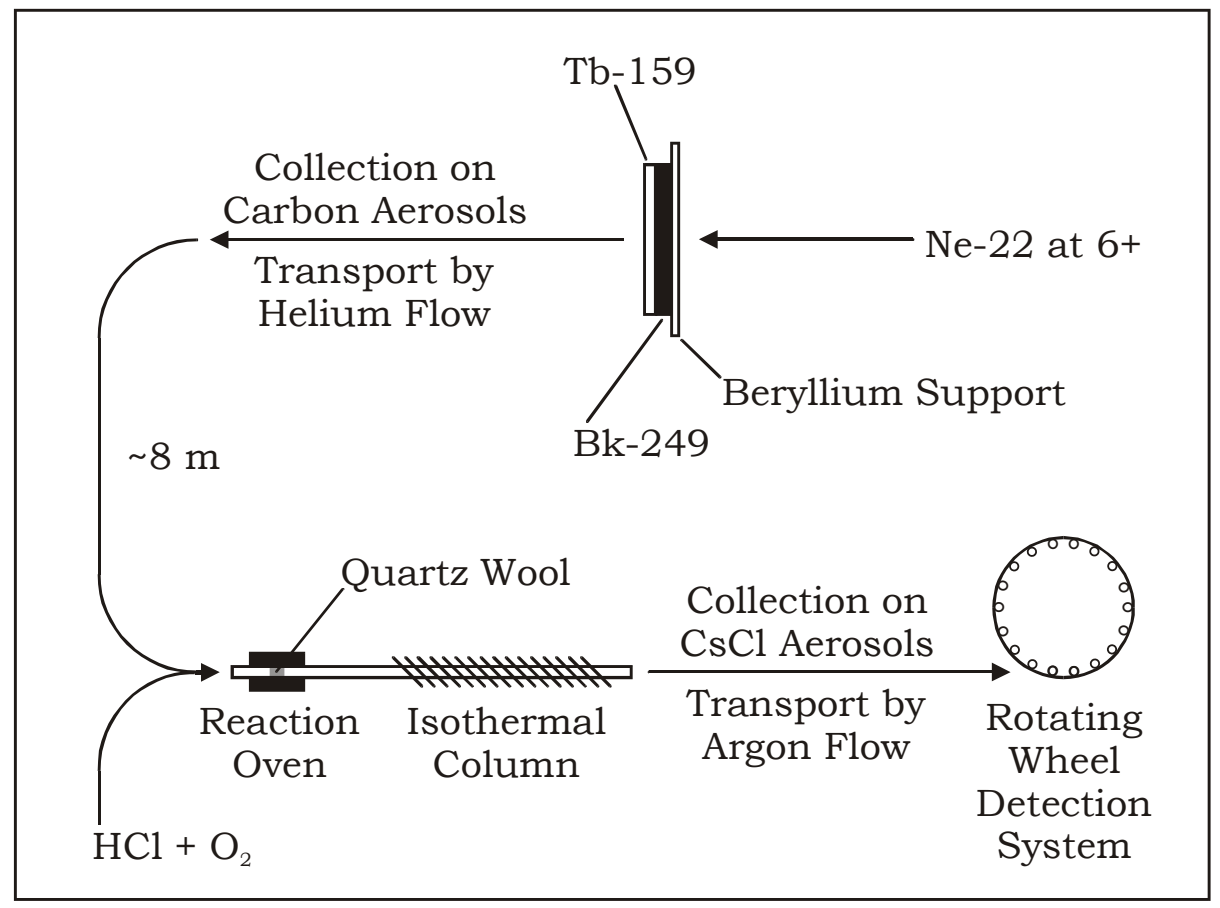

Two $\alpha$-SF chains, two $\alpha-\alpha$ chains, and one $\alpha-\alpha-\alpha$ decay chain, all attributed to the decay of ${ }^{267} \mathrm{Bh}$, were observed at $180^{\circ} \mathrm{C}$. At $150^{\circ} \mathrm{C}$ two $\alpha$-SF chains were observed, and at $75^{\circ} \mathrm{C}$ no events were observed. Random correlations should not significantly contribute to the number of $\alpha$-SF or $\alpha-\alpha-\alpha$ chains seen, but 1.2 of the 2 $\alpha-\alpha$ correlations seen are expected to be due to random correlations.

During Fall 2000, a gas chemistry experiment was run to try to further identify ${ }^{266} \mathrm{Bh}$. The parameters of this experiment were: $10^{18} 121-\mathrm{MeV},{ }^{22} \mathrm{Ne}^{6+}$ ions on target with an average current of 250 enA. The energy was chosen on the basis of my previous work [Wilk 2000] to maximize the production of ${ }^{266} \mathrm{Bh}$. The reaction oven 
was run at approximately $1050{ }^{\circ} \mathrm{C}$ with the isothermal chromatography column at $230{ }^{\circ} \mathrm{C}$ to remove any obscuring contaminants that are not volatile at this temperature. The same stepping times were used. The daughter mode was initiated for events between 8.8 and 9.8 MeV. During this experiment no ${ }^{266} \mathrm{Bh}$ was observed. In order fort this experiment to be sensitive to a bohrium isotope with a one second half-life, the cross section would have to be thirty times larger than that of ${ }^{267} \mathrm{Bh}$ due to the five second transport time through the apparatus.

\subsubsection{Results and Discussion}

The unambiguous identification of Bh after chemical separation allows us to conclude that, like its lighter homologues, Bh forms a volatile oxychloride compound, presumably $\mathrm{BhO}_{3} \mathrm{Cl}$, and behaves like a typical group seven element, but is less volatile.

Assuming the $\mathrm{Bh}$ compound to be $\mathrm{BhO}_{3} \mathrm{Cl}$ and applying a microscopic model of the adsorption-desorption process [Zvára 1982] and using the Monte Carlo code developed by Türler to fit the measured volatility curves, the evaluated standard adsorption enthalpy $\left(\Delta \mathrm{H}_{\mathrm{ads}}\right)$ of $\mathrm{BhO}_{3} \mathrm{Cl}$ on the quartz surface was determined to be $-75_{-6}^{+9} \mathrm{~kJ} / \mathrm{mol}$. The adsorption enthalpies for $\mathrm{TcO}_{3} \mathrm{Cl}$ and $\mathrm{ReO}_{3} \mathrm{Cl}$ are $-51_{-3}^{+3}$ and $-61_{-3}^{+3} \mathrm{~kJ} / \mathrm{mol}$ respectively [Eichler 2000d].

After the quartz column, $\mathrm{BhO}_{3} \mathrm{Cl}$ was transported to ROMA on $\mathrm{CsCl}$ aerosols. Unlike $\mathrm{ReO}_{3} \mathrm{Cl}, \mathrm{TcO}_{3} \mathrm{Cl}$ is so volatile that it can not be transported with $\mathrm{CsCl}$, which again indicates $\mathrm{Bh}$ is more like Re than Tc. 


\section{Chapter 6: Conclusions and Future Work}

The half-life of ${ }^{224} \mathrm{~Pa}$ was determined to be $855 \pm 19 \mathrm{~ms}$ by measuring its $\alpha$-decay using our MG system at the LBNL 88-Inch Cyclotron. New neutron-rich isotopes, ${ }^{267} \mathrm{Bh}$ and ${ }^{266} \mathrm{Bh}$, were produced in bombardments of a ${ }^{249} \mathrm{Bk}$ target and their decay was also observed using the MG system. Determination of the decay properties of these isotopes was essential for the chemical study of the group five and seven elements. The half-life and production cross-section of ${ }^{224} \mathrm{~Pa}$, were needed to determine the operational characteristics of SISAK when investigating group elements. The discovery of a long-lived isotope of bohrium was essential to the first successful chemical investigation of bohrium. All the previously identified isotopes had half-lives that were much too short for chemical investigations.

There is still work to be done on these two isotopes of ${ }^{266} \mathrm{Bh}$ and ${ }^{267} \mathrm{Bh}$. Determination of the decay properties of a shorter-lived ${ }^{266} \mathrm{Bh}$ might still be possible by using an even shorter stepping time with the MG. With some work, it should be possible to optimize the system to allow for steps as short as $0.5 \mathrm{~s}$. One of the five decays observed from ${ }^{267} \mathrm{Bh}$ has a considerably lower energy than the others, indicating the possibility of two $\alpha$-branches. Another experiment to investigate this behavior would be useful. In addition, nothing is known currently about the SF branch of these two isotopes, and an investigation of this property, due to their proximity to the deformed $\mathrm{N}=162$ neutron shell, would be especially helpful in evaluating theoretical predications.

The chemical behavior of hahnium, Ha (element 105), was investigated using 
the fast extraction system SISAK. The extraction of 0.85 -s Pa indicated an efficiency of the on-line extraction to be only $2.5 \%$, too low to observe the extraction of hahnium if it behaves similarly. However, the extraction and detection of protactinium was a success for SISAK and illustrated that this apparatus will be useful for transactinide investigations if the proper chemical system is developed and the detection sensitivity is increased.

The chemical behavior of oxychloride compounds of bohrium was investigated by isothermal gas adsorption chromatography in a quartz column at 180,150 , and $75^{\circ} \mathrm{C}$. It was found to be less volatile than the corresponding compounds of the lighter group seven homologues, rhenium and technetium. This was the first ever chemical investigation of this element, and was the first evidence for placing it in the seventh group of the periodic table.

The recent success of SISAK experiments at LBNL could not have occurred without the use of the BGS as a pre-separator to remove interfering $\alpha$-emitting isotopes. However, the BGS was not built for use as a pre-separator, but was optimized for symmetric reactions such as $\mathrm{Kr}+\mathrm{Pb}$. Asymmetric reactions like $\mathrm{Ne}+\mathrm{Bk}$ are needed for chemistry experiments due to their large production cross sections. However, these reactions suffer from low transport efficiency through the BGS. In addition, due to the superheavy element discovery program that requires low background activity, it is not possible to use radioactive targets such as berkelium in the BGS. A considerably simpler device that utilizes a single, strong gradient magnet could work as well or better. All that is needed is a magnetic filter that acts as a beam dump as well as removes the majority of unwanted contamination. Such a device 
could be built at other facilities much easier than a device such as the BGS. It might be possible to use a higher internal pressure in such a device, which would allow for a thinner interface window in the RTC, or perhaps no window at all. A dedicated chemistry pre-filter such as this would also allow the use of radioactive targets.

The SISAK system is being aggressively developed and improved. Now that it has been used to successfully separate and positively identify a transactinide element (Rf), it will not be long before it is used to actually study the difference between the transactinides and the lighter homologues in a variety of chemical systems. There are now plans to try SISAK again for seaborgium [Johansson 2001]. Work on a better continuous, on-line liquid chromatography system for seaborgium is progressing. Recently some headway toward an appropriate resin/solvent system that will give good separation between tungsten and molybdenum has also been made [Pfrepper 2000]. Now that we have a long-lived isotope of bohrium available, it is also possible to use SISAK for investigation of this element. Work on an appropriate separation system has already begun [Malmbeck 2000].

The experiments to date concerning the solution chemistry of rutherfordium have been inconclusive due to the presence of HF (a strong complexer) in the chemistry. An extraction with pure $\mathrm{HCl}$ or pure $\mathrm{HNO}_{3}$ will alleviate the ambiguity that has plagued liquid chemistry experiments on rutherfordium so far. Some work on an appropriate system has recently been performed [Sudowe 2001].

The exploration of heavy element gas chemistry is expected to be extended to hassium (element 108) this year at PSI. Dr. Uwe Kirbach has developed a thermochromographic separation apparatus [Kirbach 2001] and has performed studies 
of the homologue $\mathrm{OsO}_{4}$ [Düllmann 2001a \& 2001b, Kirbach $2000 \& 2001$ ] for the investigation of $\mathrm{HsO}_{4}$. Development of a new generation of gas chromatography devices, such as this, extends the possibility of chemical studies to even heavier elements.

The last three years at LBNL with the heavy element group, have been incredibly exciting. As Al Ghiorso has mentioned often, it is a shame that Dr. Seaborg is still not here to enjoy the success we have had. When Dr. Seaborg began his heavy element studies on plutonium 60 years ago, he never thought we could come so far! The coming years have the potential for being even more exciting as chemical investigations are extended to elements 108 and 109, and nuclear studies reveal even longer-lived isotopes of elements 110 and beyond. 


\section{Appendices}

\section{Appendix A: Glossary of Acronyms}

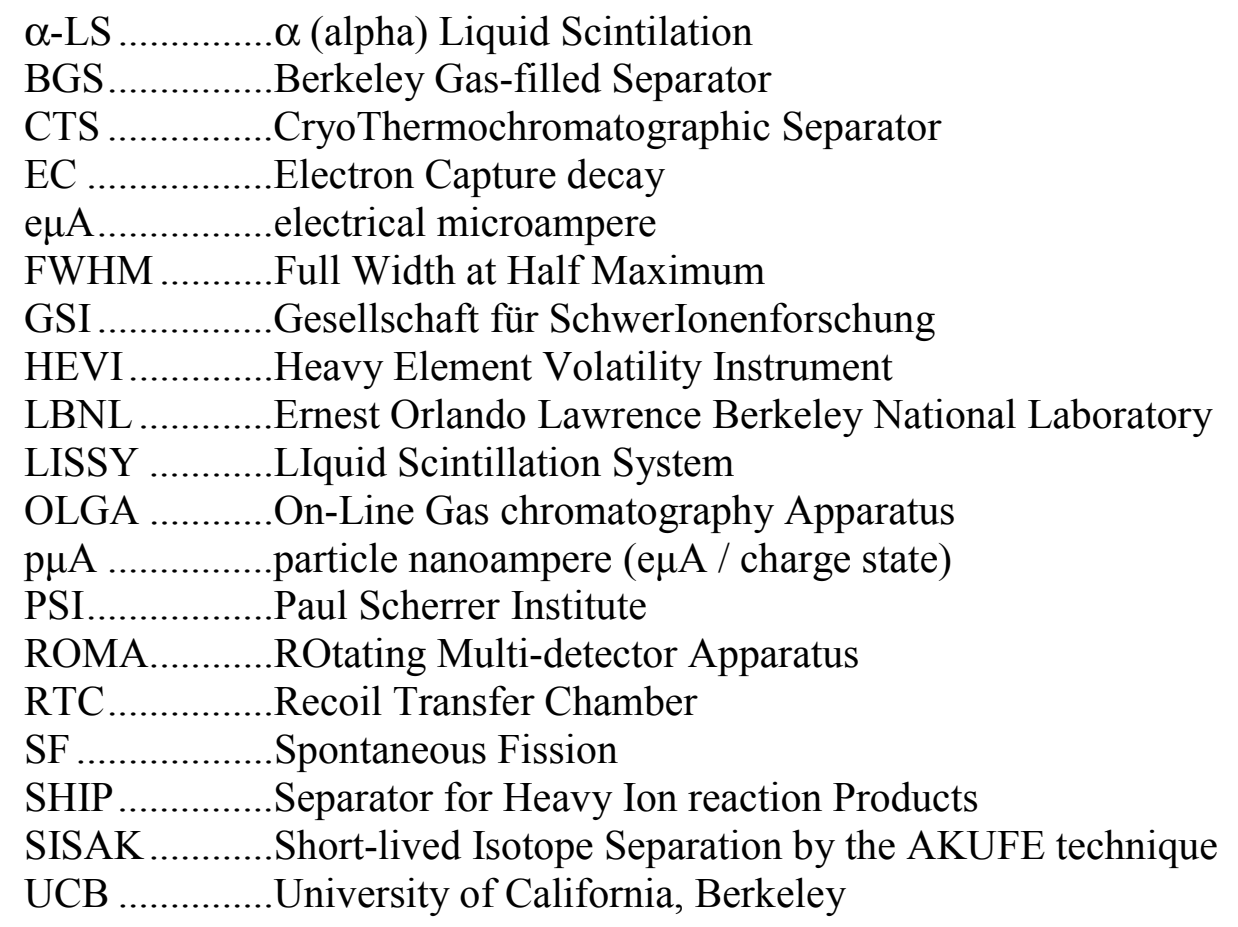




\section{Appendix B: List of primary and contributed publications and talks}

\section{B.1 Publications}

1. D. A. Shaughnessy, K. E. Gregorich, M. R. Lane, C. A. Laue, D. M. Lee, C. A. McGrath, D. A. Strellis, E. R. Sylwester, P. A. Wilk, D. C. Hoffman, "Electroncapture delayed fission probabilities ${ }^{248}$ Es and ${ }^{246}$ Es". Physical Review C (Nuclear Physics) 63, 37603 (March 2001).

2. V. Ninov, K. E. Gregorich, T. N. Ginter, F. P. Heßberger, R. Krücken, D. M. Lee, W. Loveland, W. D. Myers, J. Patin, M. W. Rowe, N. K. Seward, W. J.

Swiatecki, A. Türler and P. A. Wilk, "Production and structure of the heaviest elements". Nuclear Physics A 682, 98 (2001).

3. B. Wierczinski, K. E. Gregorich, B. Kadkhodayan, D. M. Lee, L. G. Beauvais, M. B. Hendricks, C. D. Kacher, M. R. Lane, D. A. Keeney-Shaughnessey, N. J.

Stoyer, D. A. Strellis, E. R. Sylwester, P. A. Wilk, D. C. Hoffman, R. Malmbeck, G. Skarnemark, J. Alstad, J. P. Omtvedt, K. Eberhardt, M. Mendel, A. Nähler, N. Trautmann, "Fast chemical on-line separation and detection of a subsecond $\alpha$ decaying nuclide, ${ }^{224} \mathrm{~Pa}$ ". Journal of Radioanalytical and Nuclear Chemistry 247, 57 (2001).

4. E. R. Sylwester, K. E. Gregorich, D. M. Lee, B. Kadkhodayan, A. Türler, J. L. Adams, C. D. Kacher, M. R. Lane, C. A. Laue, C. A. McGrath, D. A. Shaughnessy, D. A. Strellis, P. A. Wilk, D. C. Hoffman, "On-line gas chromatographic studies of Rf, Zr, and Hf bromides". Radiochimica Acta 88, 837 (December 2000).

5. R. Eichler, W. Brüchle, R. Dressler, C. E. Düllmann, B. Eichler, H. W. Gäggeler, K. E. Gregorich, D. C. Hoffman, S. Hübener, D. T. Jost, U. W. Kirbach, C. A. Laue, V. M. Lavanchy, H. Nitsche, J. B. Patin, D. Piguet, M. Schädel, D. A. Shaughnessy, D.A. Strellis, S. Taut, L. Tobler, Y. S. Tsyganov, A. Türler, A. Vahle, P. A. Wilk, A. B. Yakushev, "Chemical characterization of bohrium (element 107)". Nature 407, 63 (September 7, 2000).

6. P. A. Wilk, K. E. Gregorich, A. Türler, J. Adams, R. Eichler, U. W. Kirbach, M. Lane, C. A. Laue, D. Lee, V. Ninov, H. Nitsche, J. B. Patin, D. A. Shaughnessy, D. A. Strellis, and D. C. Hoffman, "Evidence for New Isotopes of Element 107: ${ }^{266}$ Bh and ${ }^{267}$ Bh". Physical Review Letters, 85, 2697 (2000). 
7. D. A. Shaughnessy, J. L. Adams, K. E. Gregorich, M. R. Lane, C. A. Laue, D. M. Lee, C. A. McGrath, J. B. Patin, D. A. Strellis, E. R. Sylwester, P. A. Wilk, D. C. Hoffman, "Electron-capture delayed fission properties of ${ }^{242}$ Es". Physical Review C (Nuclear Physics) 61, 044609 (April 2000).

8. V. Ninov, K. E. Gregorich, W. Loveland, A. Ghiorso, D. C. Hoffman, D. M. Lee, H. Nitsche, W. J. Swiatecki, U. W. Kirbach, C. A. Laue, J. L. Adams, J. B. Patin, D. A. Shaughnessy, D. A. Strellis, and P. A. Wilk, "Observation of Superheavy Nuclei Produced in the Reaction of ${ }^{86} \mathrm{Kr}$ with ${ }^{208} \mathrm{~Pb}$ ". Physical Review Letters 83, 1104 (1999).

9. C. A. Laue, K. E. Gregorich, R. Sudowe, M. B. Hendricks, J. L. Adams, M. R. Lane, D. M. Lee, C. A. McGrath, D. A. Shaughnessy, D. A. Strellis, E. R. Sylwester, P. A. Wilk, and D. C. Hoffman, "New plutonium isotope: ${ }^{231} \mathrm{Pu}$ ". Physical Review C (Nuclear Physics) 59, 3086 (1999).

10. M. R. Lane, K. E. Gregorich, D. M. Lee, B. Wierczinski, C. A. McGrath, M. B. Hendricks, D. A. Shaughnessy, D. A. Strellis, E. R. Sylwester, P. A. Wilk, and D. C. Hoffman, " ${ }_{105}{ }^{261} \mathrm{Ha}$ from the ${ }^{250} \mathrm{Cf}\left({ }^{15} \mathrm{~N}, 4 n\right)$ and ${ }^{243} \mathrm{Am}\left({ }^{22} \mathrm{Ne}, 4 n\right)$ reactions". Physical Review C 58, 3413 (1998).

11. P. A. Wilk, H. F. VanBrocklin, T. F. Budinger, "Photonuclear Production of PET Isotopes". to be published (1998).

12. P. A. Wilk, K. E. Gregorich, M. B. Hendricks, M. R. Lane, D. M. Lee, C. A. McGrath, D. A. Shaughnessy, D. A. Strellis, E. R. Sylwester, and D. C. Hoffman, "Improved Half-life Measurement of ${ }^{224} \mathrm{~Pa}$ and its ${ }^{209} \mathrm{Bi}\left({ }^{18} \mathrm{O}, 3 n\right)^{224} \mathrm{~Pa}$ Production Cross Section”. Physical Review C 56, 1626-1628 (1997) and Erratum, Physical Review C 58, 1352 (1998).

13. P. A. Wilk, K. E. Gregorich, M. B. Hendricks, M. R. Lane, D. M. Lee, C. A. McGrath, D. A. Shaughnessy, D. A. Strellis, E. R. Sylwester, and D. C. Hoffman, "Improved Half-life Measurement of ${ }^{224} \mathrm{~Pa}$ and its ${ }^{209} \mathrm{Bi}\left({ }^{18} \mathrm{O}, 3 n\right){ }^{224} \mathrm{~Pa}$ Production Cross Section”. LBNL Annual Report (1996).

14. Philip Wilk, "Nuclear Structure of ${ }^{263}$ Sg", unpublished (1996).

15. Philip Wilk, "Time Resolved Resonance Raman Spectroscopy of Chromium Pentacarbonyl - or: how I learned to stop worrying and love the wave equation". Reed College Thesis (1995). 


\section{B.2 Selected Abstracts}

1. A.W. Saunders, E.A, Kanpp, W.P. Trower, P.A. Wilk, H.F. VanBrocklin, T.F. Budinger, "Photoproduction of PET radioisotopes revisited". European Conference on Accelerator Applications (ECAART5), Netherlands, 1997.

2. B. Wierczinski, J. Alstad, K. Eberhardt, K.E. Gregorich, M.B. Hendricks, D.C. Hoffman, D.A. Keeney, M.R. Lane, D.M. Lee, R. Malmbeck, J.P. Omtvedt, G. Skarnemark, D.A. Strellis, E.R. Sylwester, N. Trautmann, P.A. Wilk, "Investigation of the Complexation Behavior of Transactinides". 4th International Conference on Nuclear and Radiochemistry, St. Malo, France, September, 1996.

\section{B.3 Talks and Lectures}

1. P. A. Wilk, K. E. Gregorich, H. Nitsche, D. C. Hoffman, "First Successful Chemistry of Bohrium". Department of Energy, Basic Energy Sciences, Heavy Element Contractors' Meeting, Argonne National Laboratory, November, 2000.

2. P. A. Wilk, K. E. Gregorich, A. Tuerler, J. Adams, R. Eichler, U. W. Kirbach, M. Lane, C. A. Laue, D. Lee, V. Ninov, H. Nitsche, J. B. Patin, D. A. Shaughnessy, D. A. Strellis, and D. C. Hoffman, "New isotopes of element 107: ${ }^{266} \mathrm{Bh}$ and ${ }^{267}$ Bh". American Chemical Society, San Francisco National Meeting, April 2000.

3. P. A. Wilk, K. E. Gregorich, M. B. Hendricks, M. R. Lane, D. M. Lee, C. A. McGrath, D. A. Shaughnessy, D. A. Strellis, E. R. Sylwester, and D. C. Hoffman, "Improved Half-life Measurement of ${ }^{224} \mathrm{~Pa}$ and its ${ }^{209} \mathrm{Bi}\left({ }^{18} \mathrm{O}, 3 n\right){ }^{224} \mathrm{~Pa}$ Production Cross Section”. American Chemical Society, San Francisco National Meeting, 1997.

4. Philip Wilk, "Properties and Production of Protactinium-224". Ph.D. Candidate, Chemistry Department, UC Berkeley, October 1996. 


\section{Appendix C: Review Articles and General Texts on Nuclear Chemistry}

Darleane C. Hoffman, “The Heaviest Elements”. Chemistry and Engineering News 72, 24 (1994).

Darleane C. Hoffman and Diana M. Lee, "Chemistry of the Heaviest Elements One Atom at a Time". Journal of Chemical Education 76, 331 (1999).

Gottfried Münzenberg, "Discoveries of the Heaviest Elements". Journal of Physics G: Nuclear Particle Physics 25, 717-725 (1999).

Valeria G. Pershina, "Electronic Structure and Properties of the Transactinides and Their Compounds”. Chemical Reviews 96, 1977-2010 (1996).

Matthias Schädel, "Chemistry of the Transactinide Elements". Radiochimica Acta 70/71, 207-223 (1995).

Glenn T. Seaborg, "Tranuranium Elements: the Synthetic Actinides". Radiochimica Acta 70/71, 69-90 (1995). 


\section{Appendix D: Photographs and Color Pictures}
D.1
SHEIKS Group 1997.
D.2
SHEIKS and SISAK 2000.
D. 3
Cave 0 electronics for the bohrium-266 experiment.
D.4
The VAX 4010 microcomputer used for data acquisition.
D. 5
Personal monitoring. Note safety equipment.
D. 6
D.7
D.8
D.9
The MG, controller, and aerosol oven for the ${ }^{266} \mathrm{Bh}$ experiment.
D. 10
D. 11
MG wheel washing and inspection during the ${ }^{266} \mathrm{Bh}$ experiment.
D.12
D. 13
The Berkeley Gas-Filled Separator.
CAD schematic of the BGS
SISAK Meander Cell, 2000.
SISAK centrifuge and detector, 2000.
The Recoil Transfer Chamber affixed to the BGS.
The RTC interface window with support grid. 


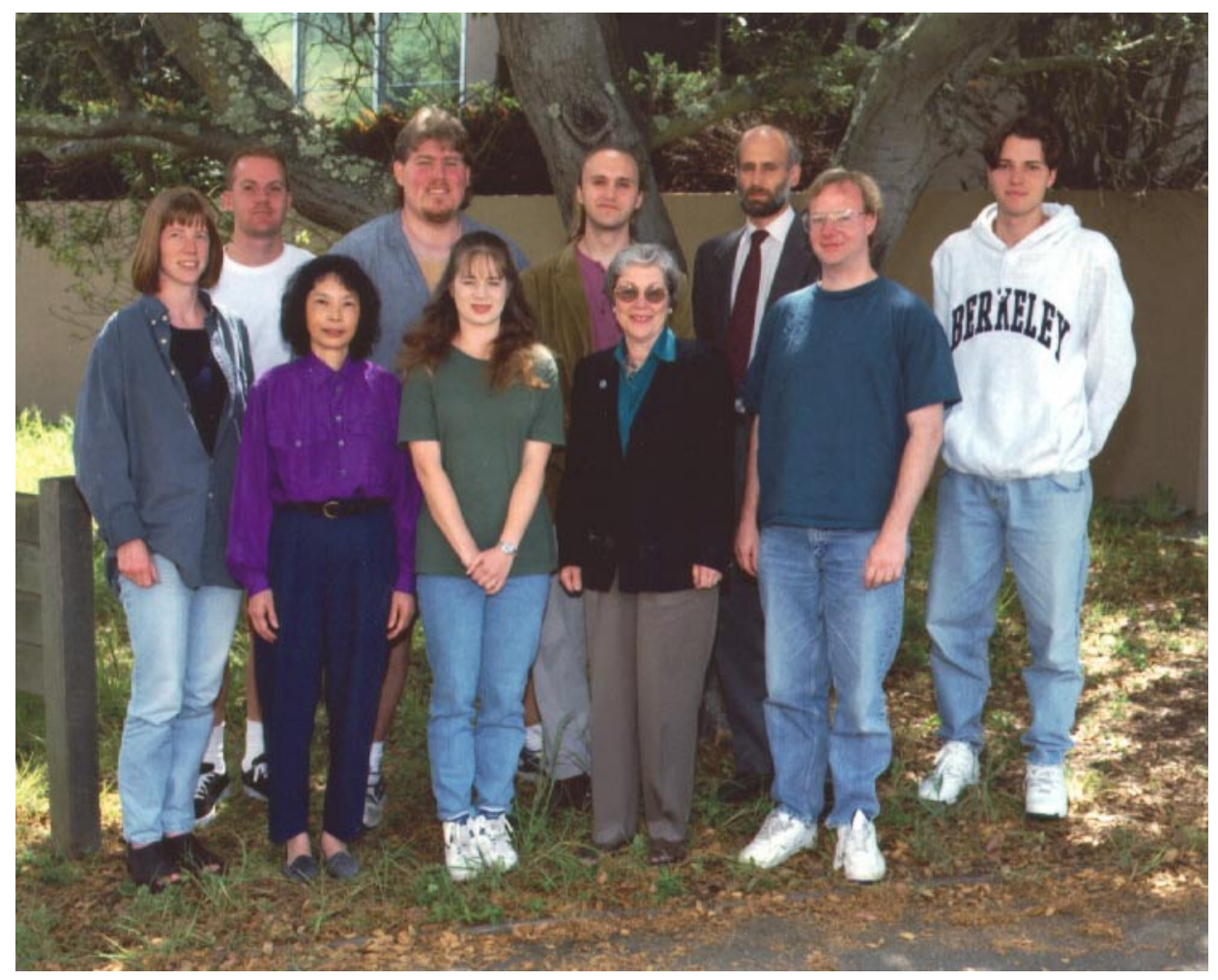

Photograph D.1: SHEIKS Group 1997

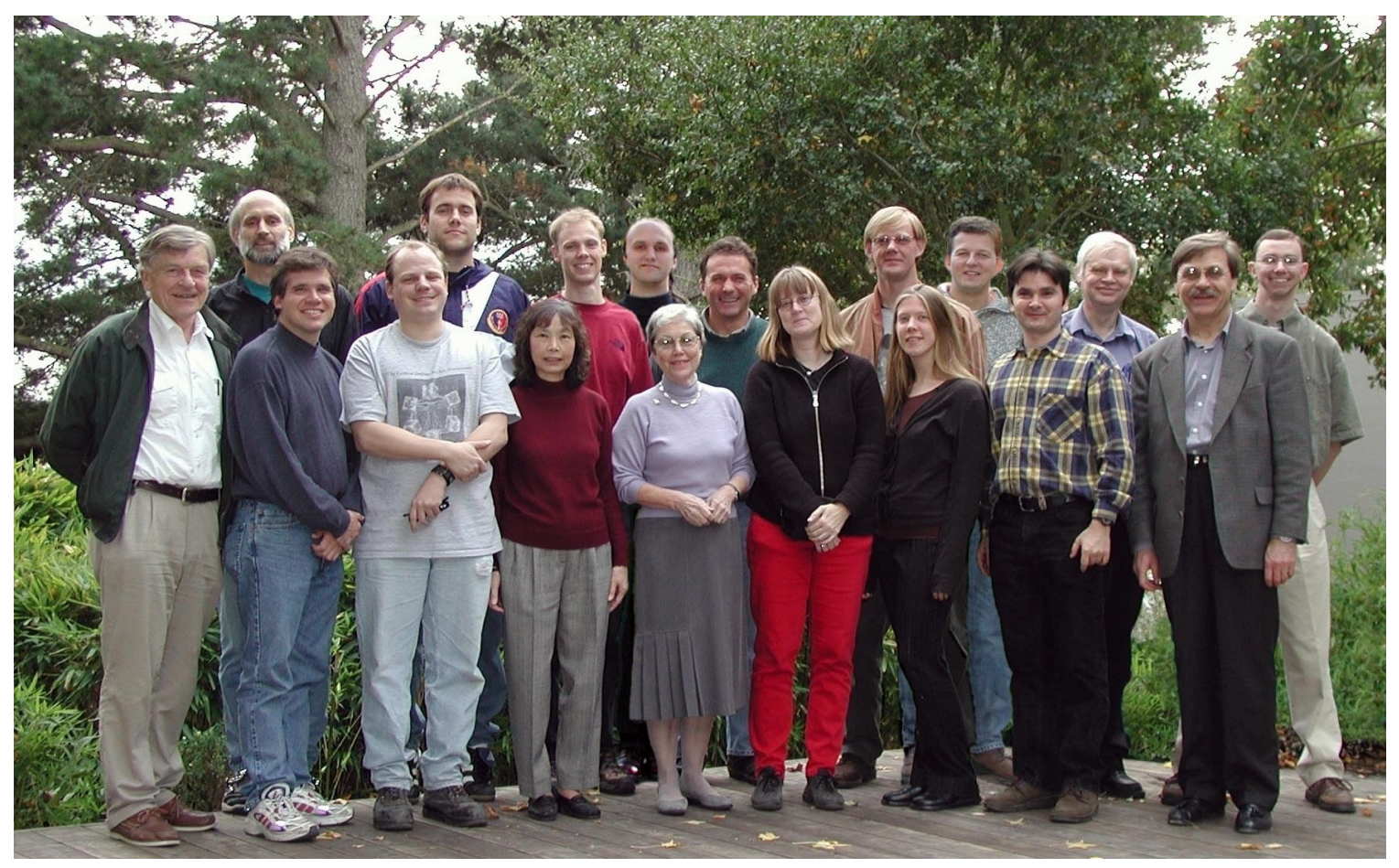

Photograph D.2: SHEIKS and SISAK 2000 


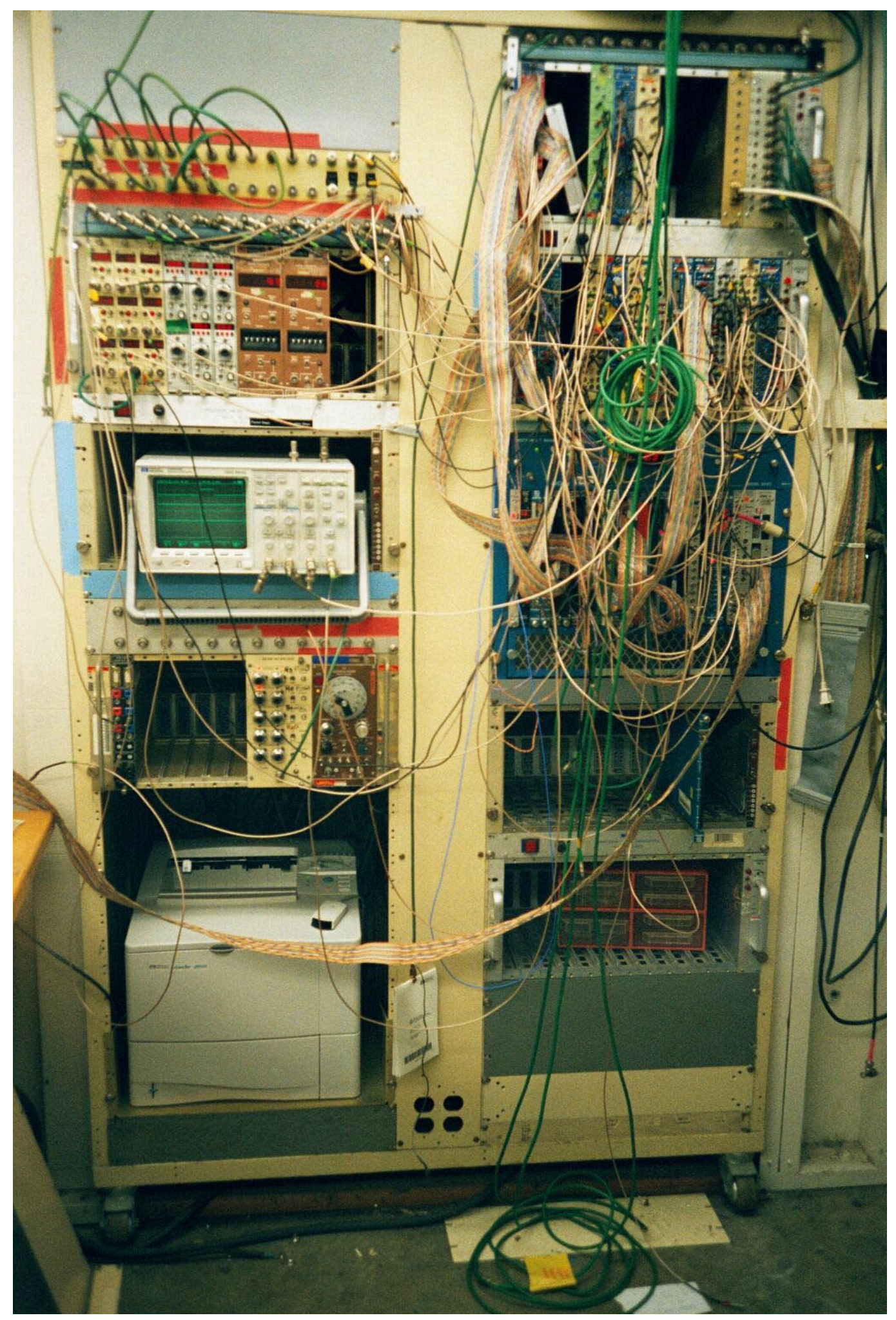

D. 3 Cave 0 electronics for the bohrium-266 experiment. 


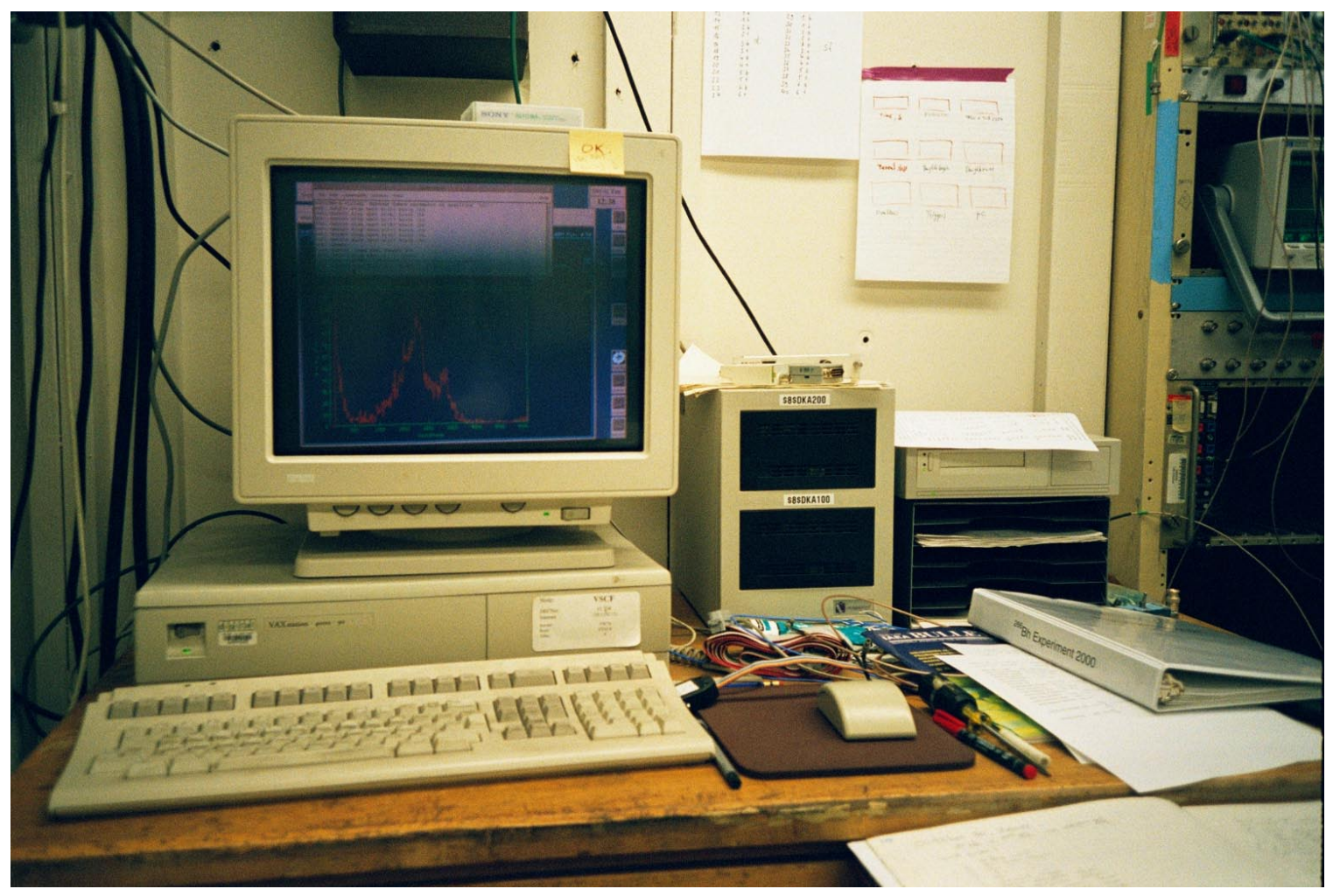

Photograph D.4: The VAX 4010 microcomputer used for data acquisition.

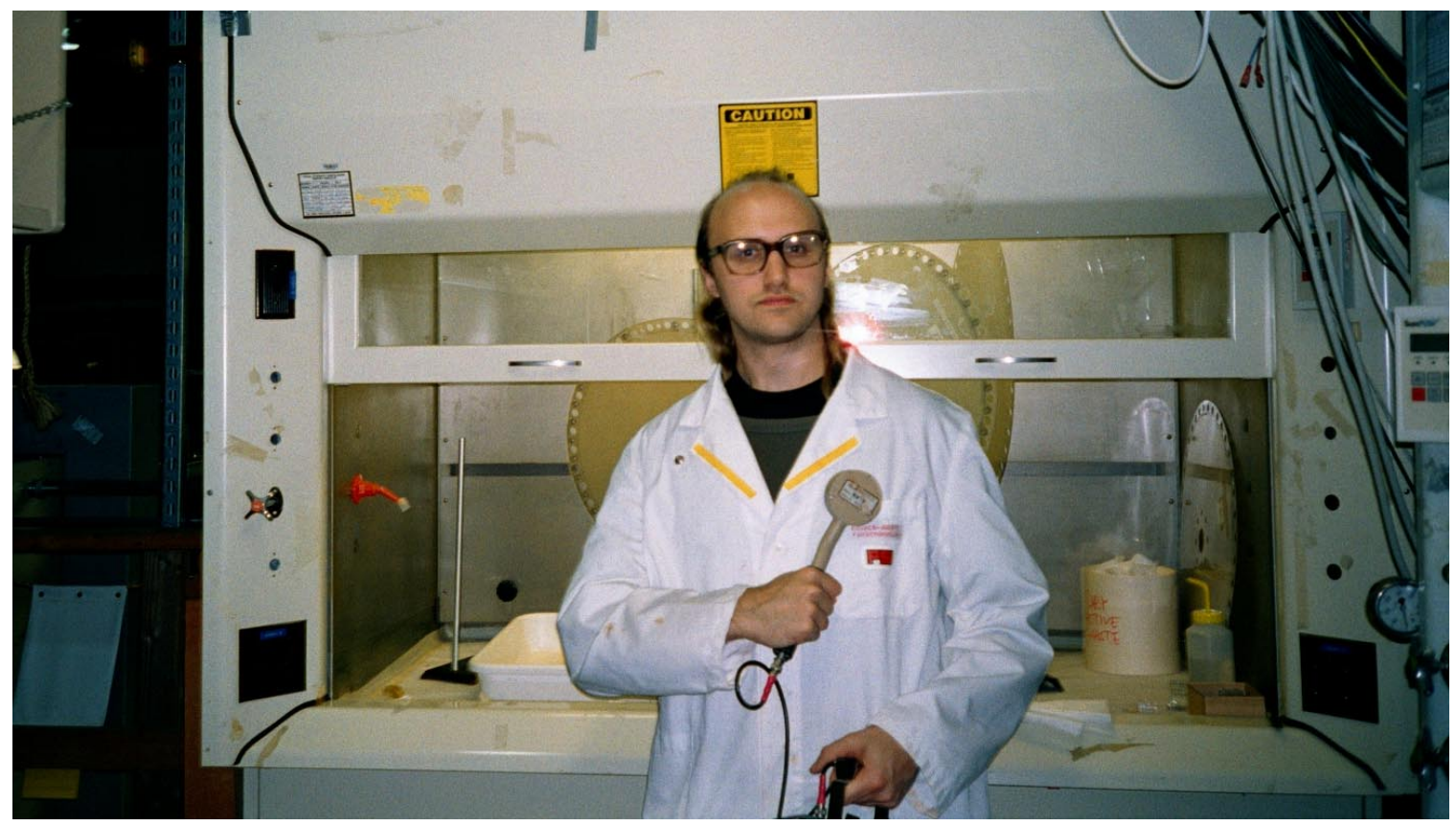

Photograph D.5: Personal monitoring. Note safety equipment. 


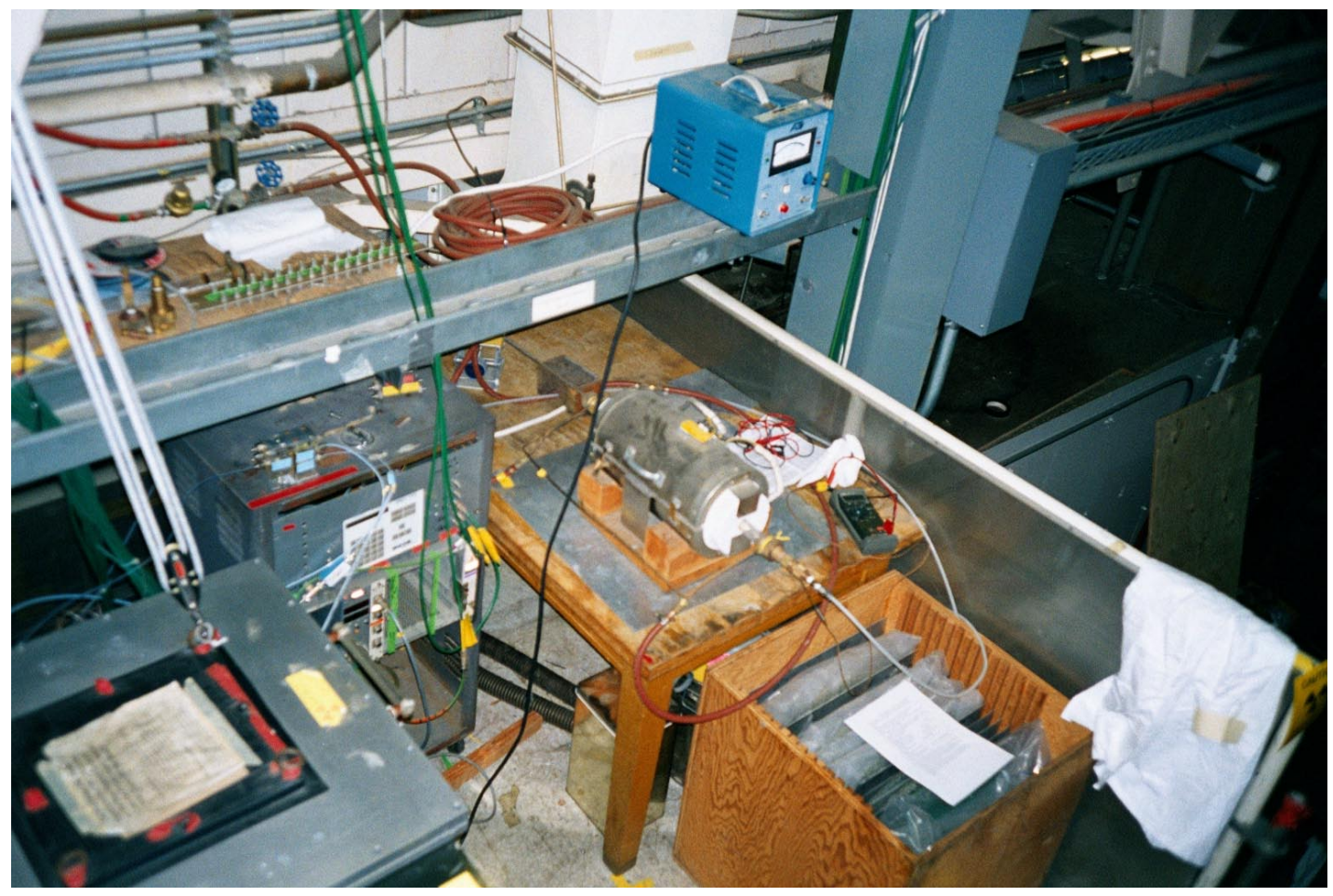

Photograph D.6: The MG, controller, and aerosol oven for the ${ }^{266} \mathrm{Bh}$ experiment.

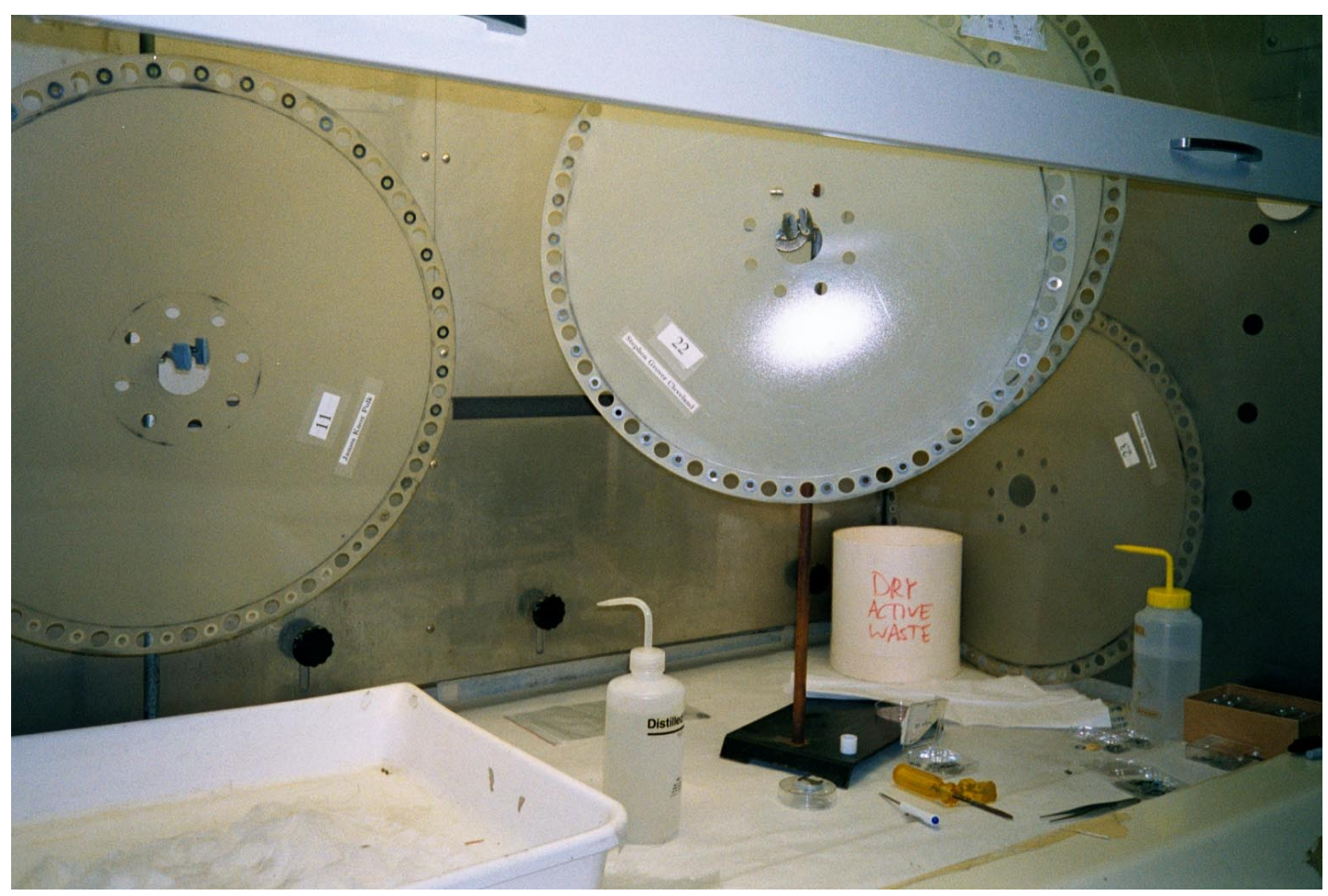

Photograph D.7: The MG wheel washing and inspection during the ${ }^{266} \mathrm{Bh}$ run. 


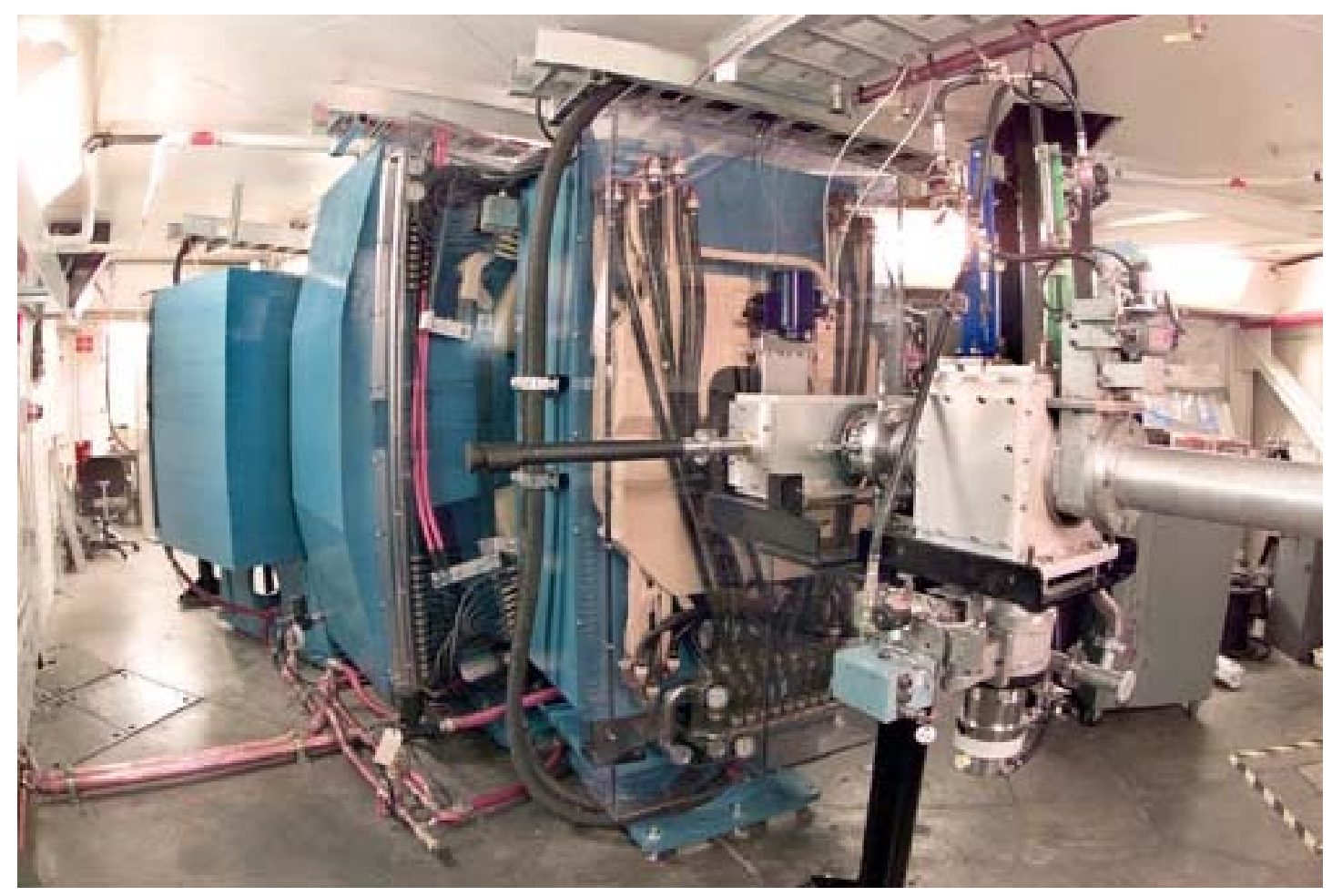

Photograph D.8: The Berkeley Gas-Filled Separator Photo by LBNL

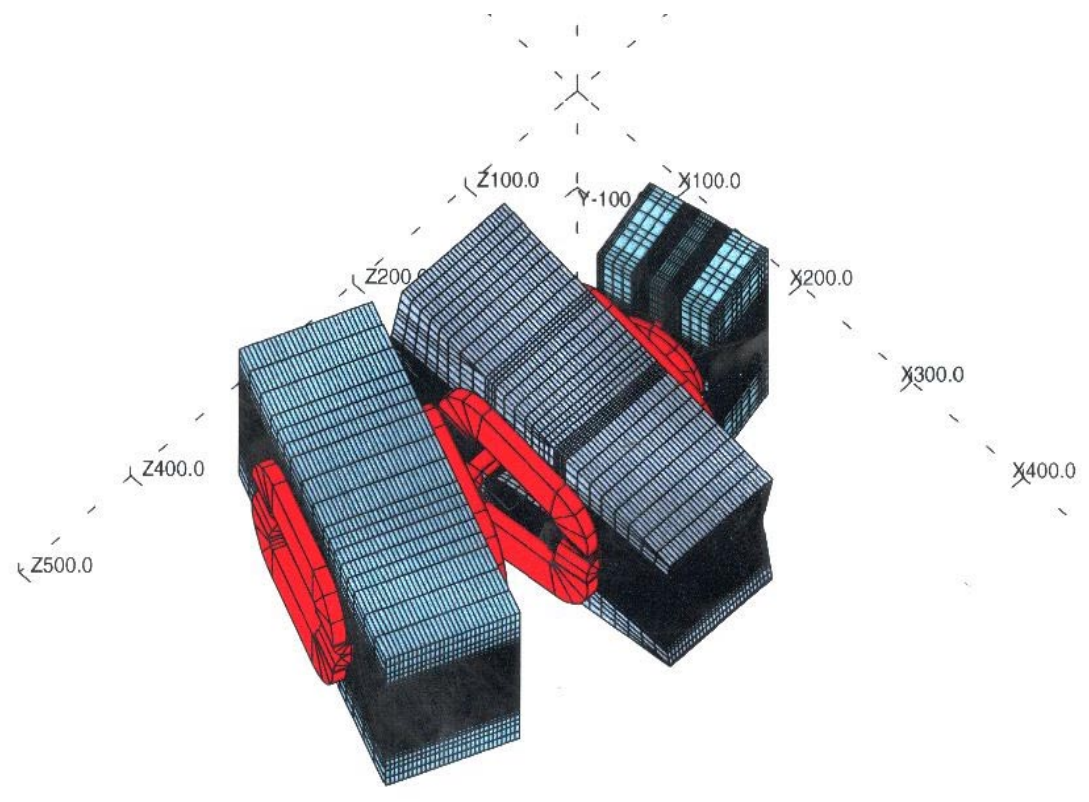

Photograph D.9: CAD schematic of the BGS Courtesy of Dr. Ken Gregorich 


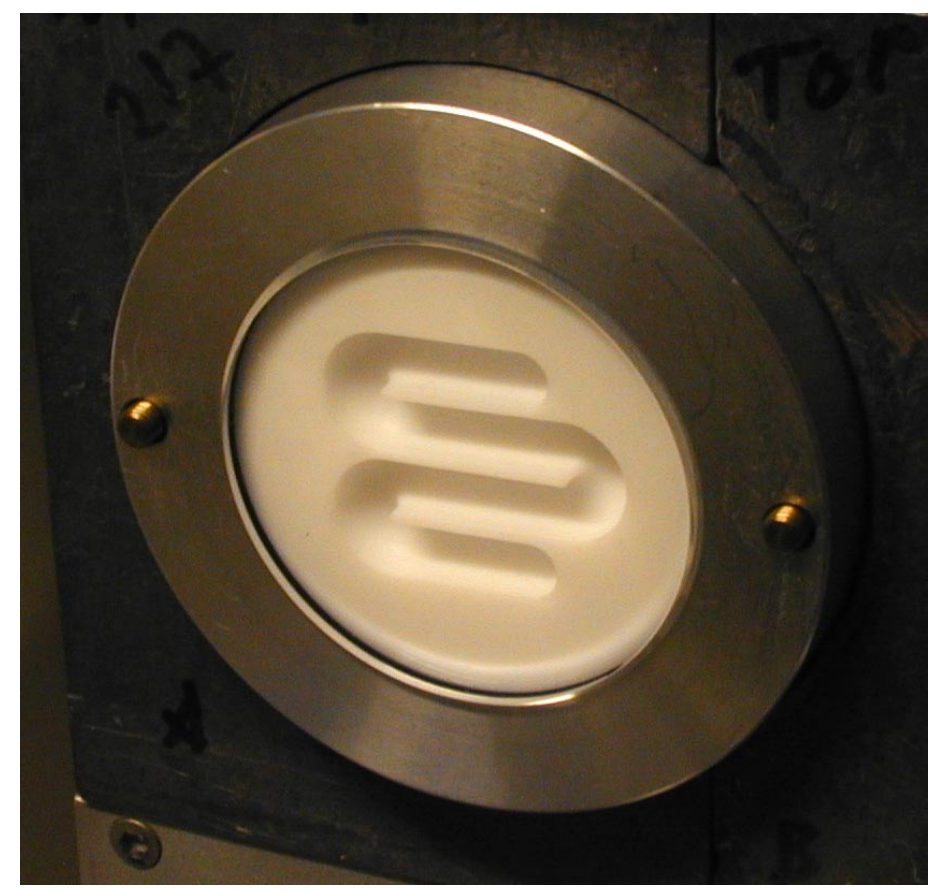

Photograph D.10: SISAK meander cell, 2000 Photo by Dr. Ken Gregorich

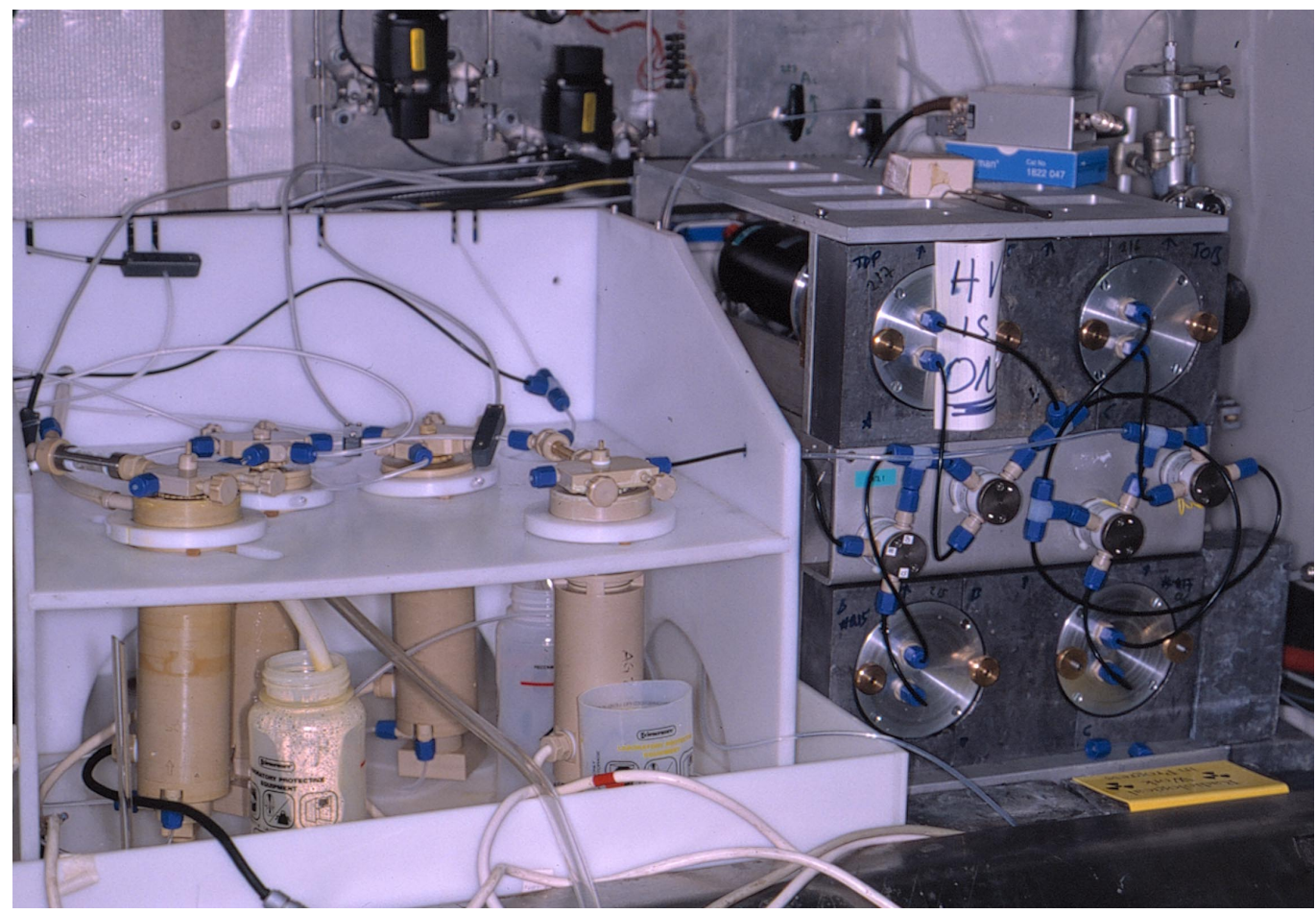

Photograph D.11: SISAK centrifuge and detector, 2000

Photo by Dr. Jan-Petter Omtvedt 


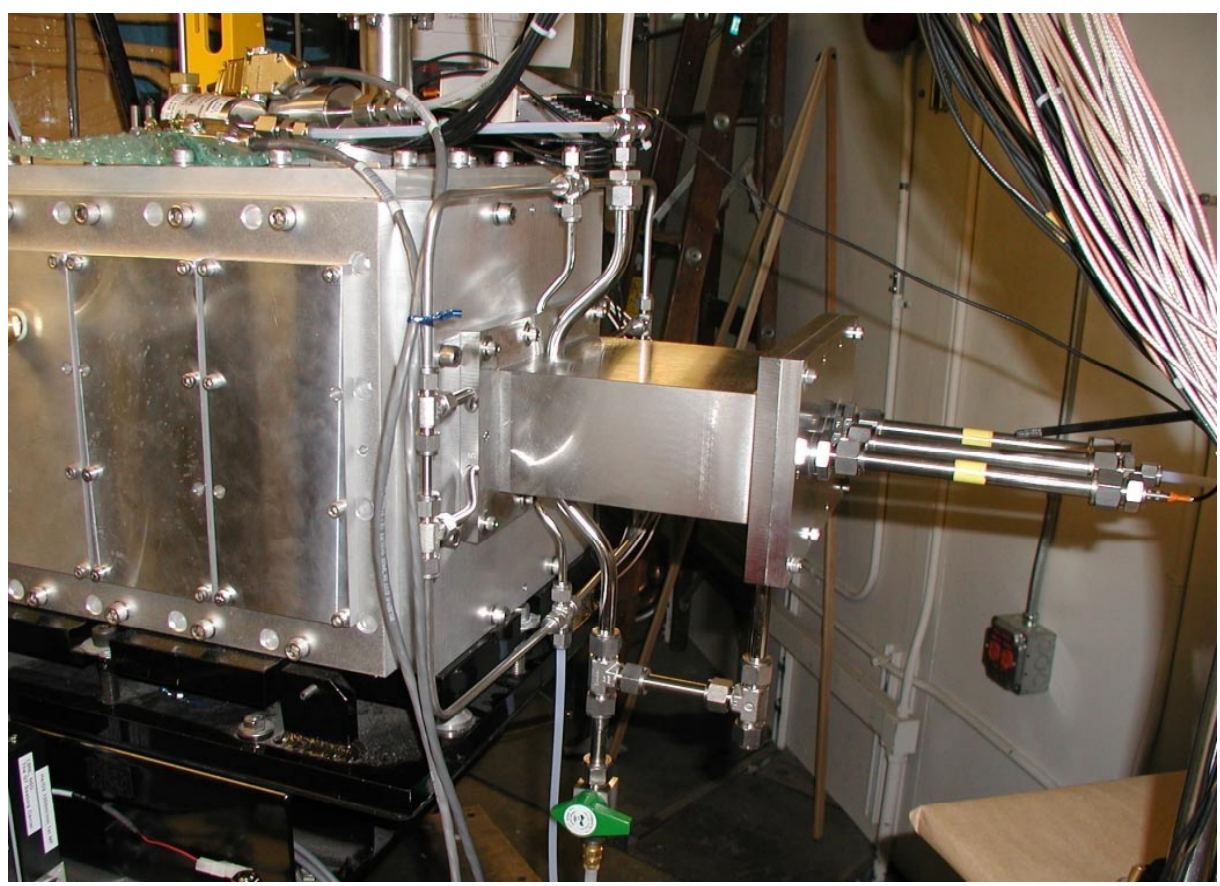

Photograph D.12: The Recoil Transfer Chamber affixed to the BGS Photo by Dr. Uwe Kirbach

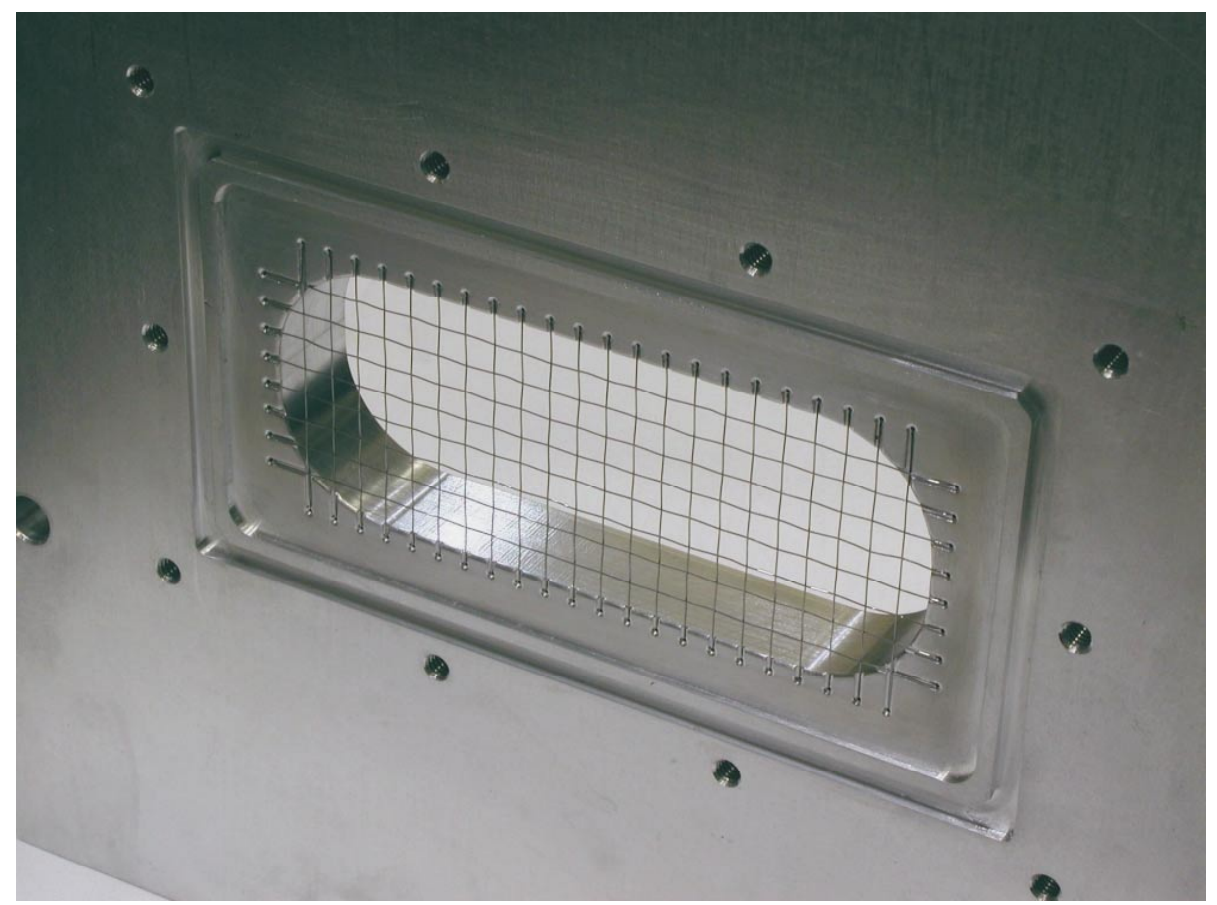

Photograph D.13: The RTC interface window with support grid Photo by Dr. Uwe Kirbach 


\section{Appendix E: Computer Code}

\section{E.1f_user.c from the CVC acquisition}

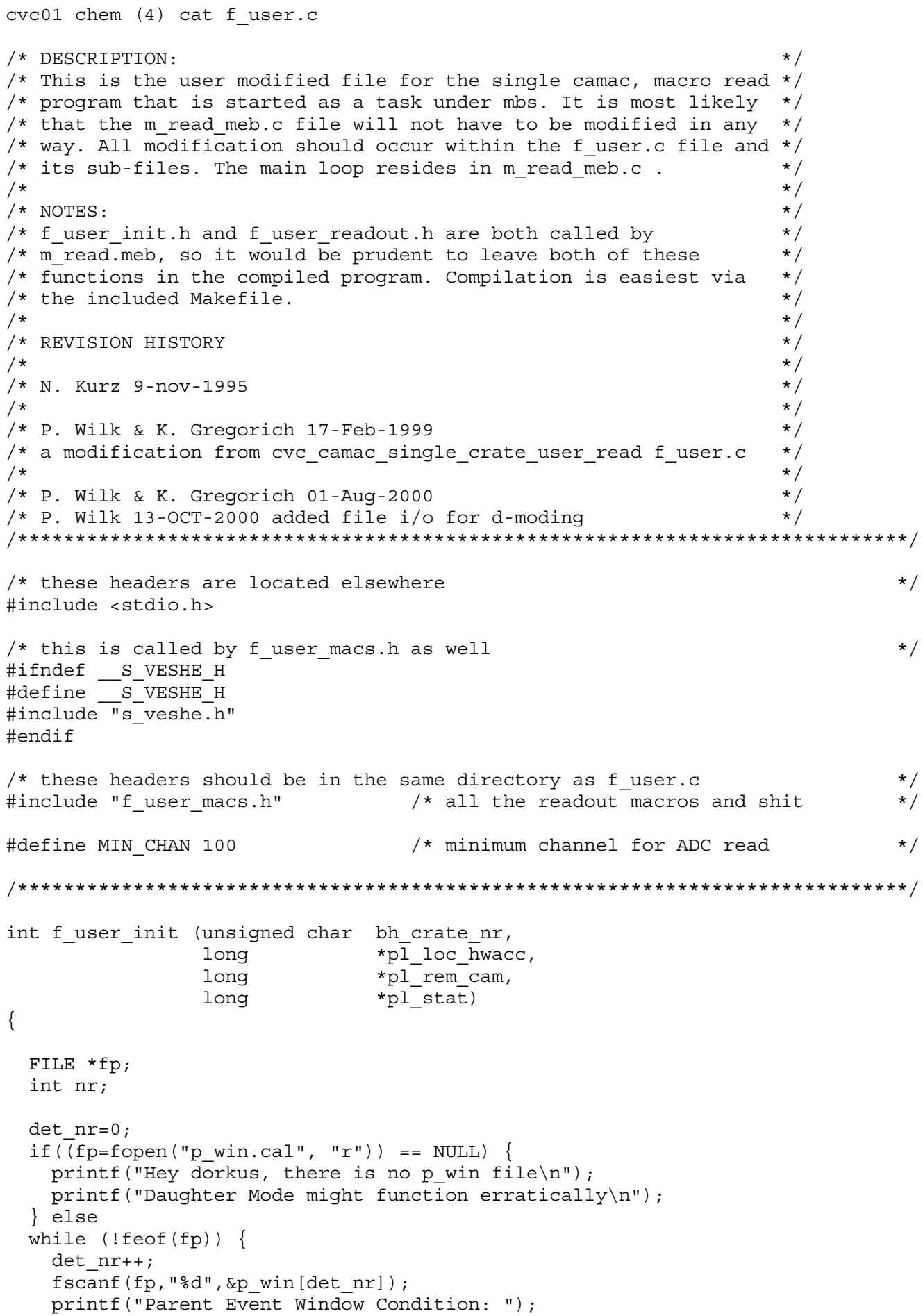




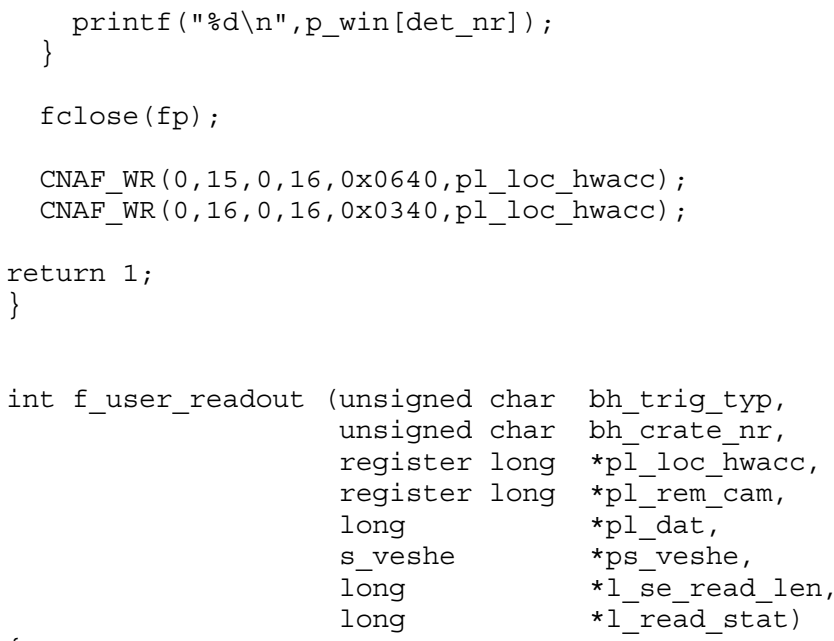




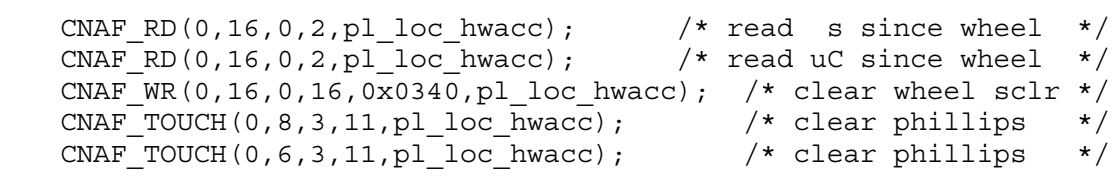

break;

case 4 :

CNAF_RD $(0,22,0,2, \mathrm{pl}$ loc hwacc);

/* begin daughter mode event */

CNAF_WR $(0,15,0,16,0 \times 0600, \mathrm{pl}$ loc_hwacc $) ; / *$ command word *

$\mathrm{CNAF}_{-}^{-} \mathrm{RD}\left(0,15,0,2, \mathrm{pl}{ }_{1} \mathrm{lOC}_{-} \mathrm{hwaCC}\right) ;-\quad$ * read us since begin *

CNAF_RD $(0,15,0,2, \mathrm{pl}$ loc_hwacC); / * read ms since begin */

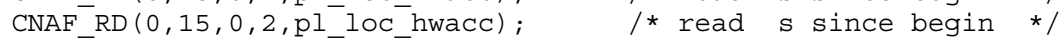

CNAF $\mathrm{RD}(0,15,0,2, \mathrm{pl}$ loc hwacC); $/$ * read uC since begin */

$\mathrm{CNAF}_{-}^{-} \mathrm{RD}\left(0,15,0,2, \mathrm{pl}{ }^{-}\right.$loc_hwacC $) ; \quad / *$ read parent steps */

CNAF_RD $(0,15,0,2, \mathrm{pl}$ loc_hwacC); /* read beg dau steps */

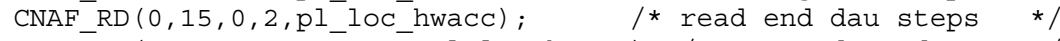

CNAF WR $(0,16,0,16,0 \bar{x} 030 \overline{0}, \mathrm{pl}$ loc hwacc $) ; / *$ command word */

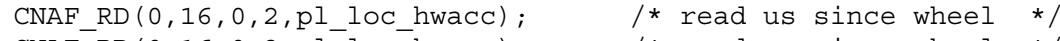

CNAF_RD $\left(0,16,0,2, \mathrm{pl}^{-}{ }^{-}{ }_{\mathrm{CO}}{ }_{-}\right.$hwacC $) ; \quad / *$ read $\mathrm{ms}$ since wheel */

CNAF_RD $\left(0,16,0,2, \mathrm{pl}_{-}\right.$loc_hwacc); / $)$read $\mathrm{s}$ since wheel */

CNAF_RD $\left(0,16,0,2, \mathrm{pl}_{-}{ }_{10 C}\right.$ hwacC $) ; \quad$ * read uC since wheel */

CNAF_WR $\left(0,16,0,16,0 \times 0340, p l \_l o c \_h w a c C\right) ; / *$ clear wheel sclr */

CNAF_TOUCH $(0,8,3,11, \mathrm{pl}$-loc_hwacC); / clear phillips */

$\operatorname{CNAF} \operatorname{TOUCH}(0,6,3,11, \mathrm{pl}$ loc hwacc) ; * clear phillips *

break;

case 8 :

CNAF RD $(0,22,0,2, \mathrm{pl}$ loc hwacc):

/* end daughter mode event */

CNAF_WR $(0,15,0,16,0 \bar{x} 060 \overline{0}, \mathrm{pl}$ loc_hwacc $) ; / *$ command word *

CNAF_RD $(0,15,0,2, \mathrm{pl}$ loc_hwacC $) ; \quad / *$ read us since begin */

CNAF_RD $\left(0,15,0,2, \mathrm{pl}_{-}\right.$loc_hwacC); / * read $\mathrm{ms}$ since begin */

CNAF_RD $\left(0,15,0,2, \mathrm{pl}^{-}\right.$loc_hwacC); $/$* read s since begin */

$\mathrm{CNAF}^{-} \mathrm{RD}(0,15,0,2, \mathrm{pl}$ loc_hwaCC $) ; \quad / *$ read uC since begin */

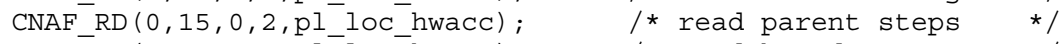

CNAF_RD $(0,15,0,2, \mathrm{pl}$ loc_hwacC); / $)$ read beg dau steps */

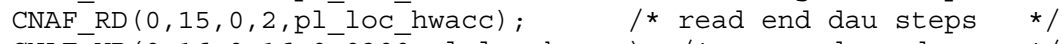

CNAF WR $(0,16,0,16,0 \bar{x} 030 \overline{0}, \mathrm{pl}$ loc hwacC $) ; / *$ command word *

$\mathrm{CNAF}_{-}^{-} \mathrm{RD}(0,16,0,2, \mathrm{pl}$ loc_hwaCC $) ; \quad / *$ read us since wheel */

CNAF_RD $(0,16,0,2, \mathrm{pl}$ loc_hwacC); /* read ms since wheel */

CNAF_RD $\left(0,16,0,2, \mathrm{pl}_{-}\right.$loc_hwacC); / $)$read s since wheel */

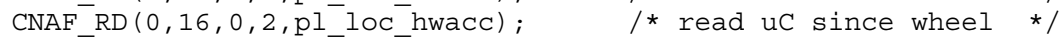

CNAF_WR $(0,16,0,16,0 \times 0340, \mathrm{pl}$ loc_hwacc); /* clear wheel sclr * /

CNAF_TOUCH $(0,8,3,11, \mathrm{pl}$ _loc_h̆wacC); $)$ * clear phillips */

$\operatorname{CNAF}$ TOUCH $(0,6,3,11, \mathrm{pl}$ _loc_hwacc); * clear phillips */

\}

break ;

\#ifdef DEBUG

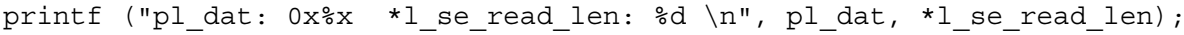
\#endif / * DEBUG */

return (9999); 


\section{E.2 GOOSY analysis}

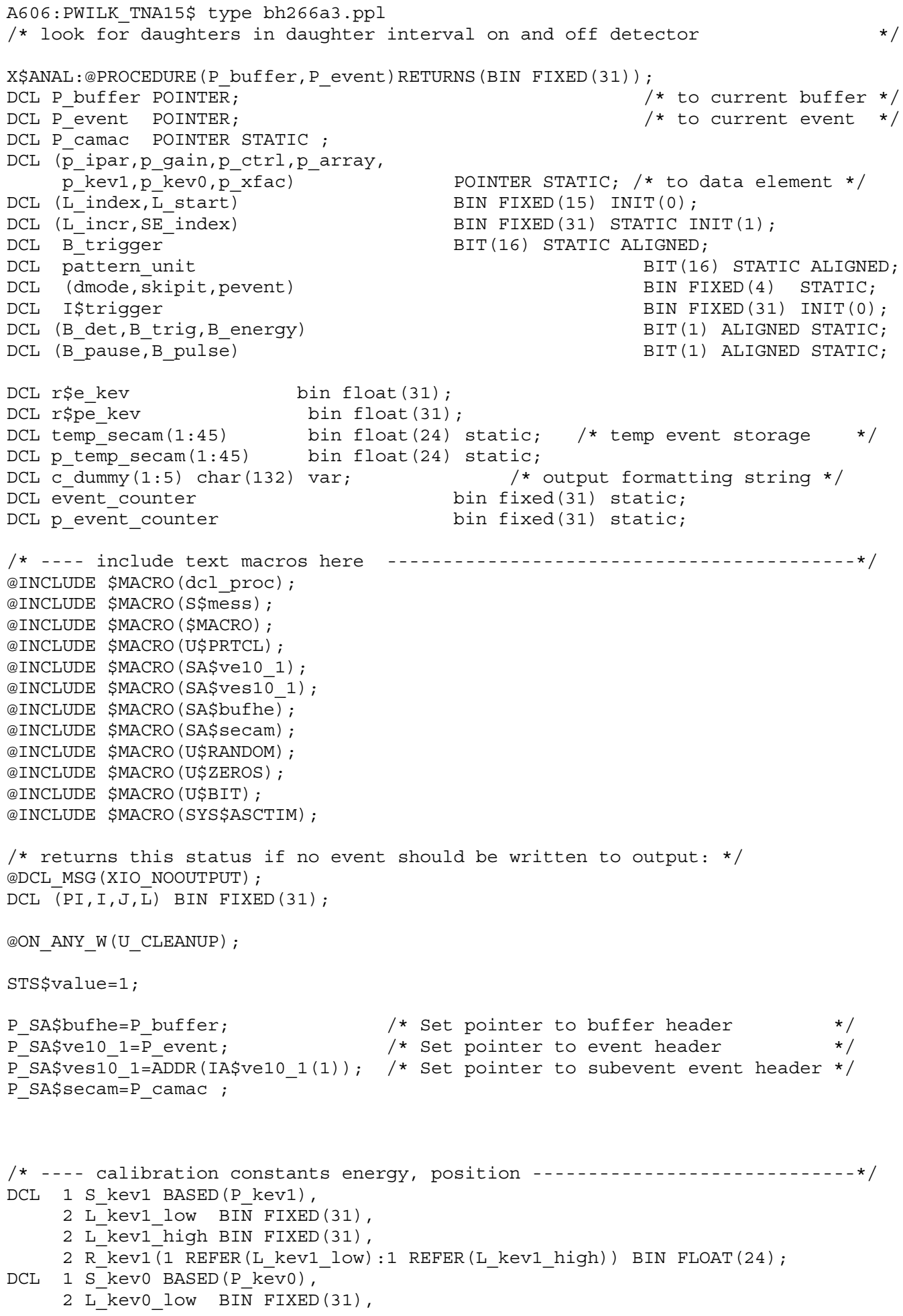


$\mathrm{DO} I=1$ to 12 ;

IF (ra\$secam $(I+28)>500)$ THEN DO ;

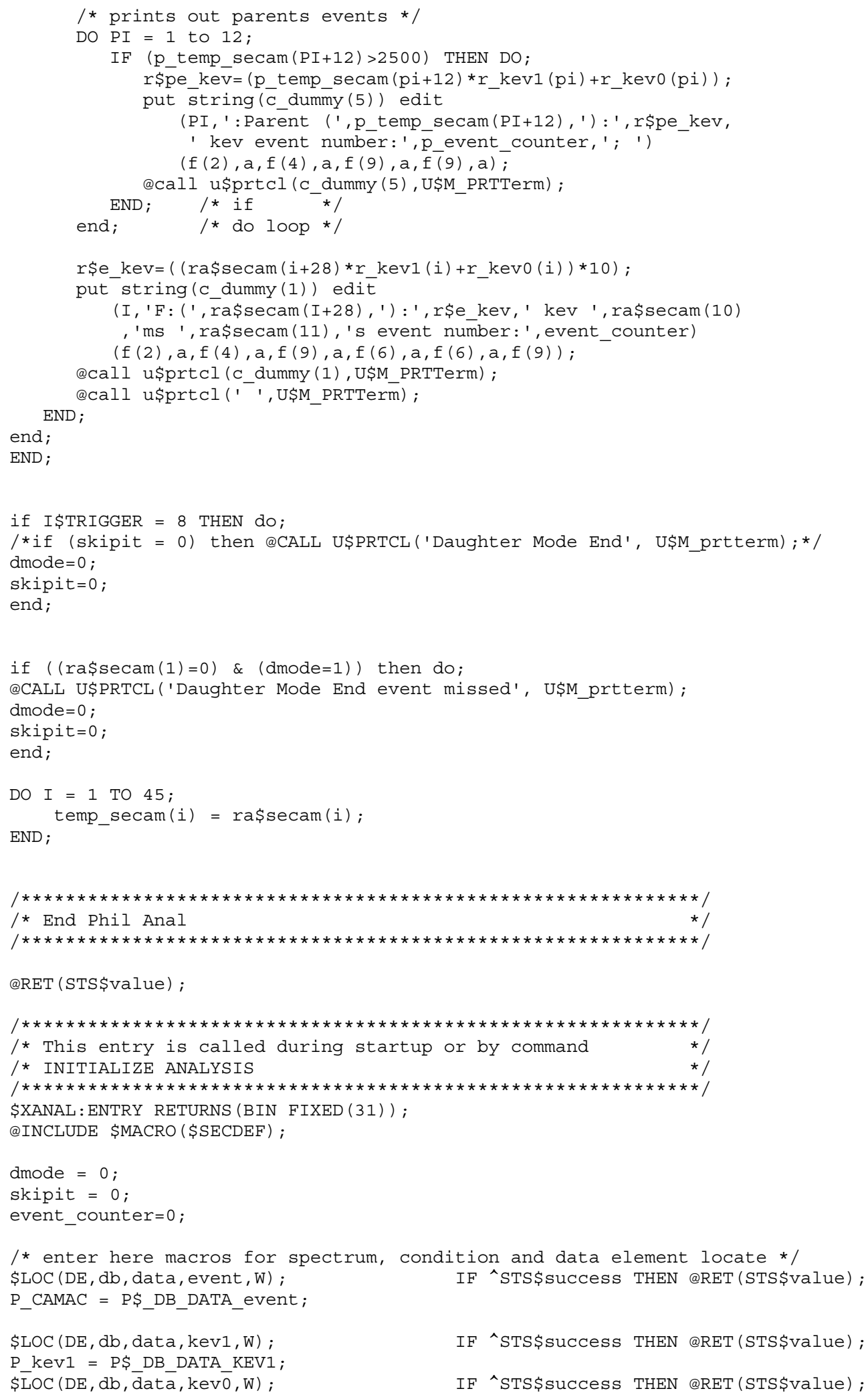


P_keV0 = P\$_DB_DATA_KEVO;

STS\$value $=1$

@RET (STS\$value)

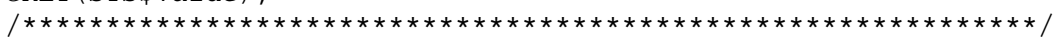

/* This routine is called in case of an error

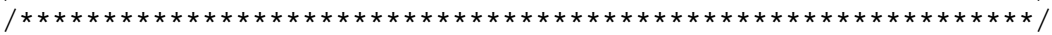

U CLEANUP : PROCEDURE;

END U_CLEANUP;

END X\$ANAL;

/** END X\$ANAL PLI Procedure

A606:PWILK_TNA15\$ 


\section{Bibliography}

[Alstad 1997] J. Alstad, J. P. Omtvedt, private communication and report (1997).

[Altzitzoglou 1990] T. Altzitzoglou, J. Rogowski, M. Skålberg, J. Alstad, G. Herrmann, N. Kaffrell, G. Skarnemark, W. Talbert, N. Trautmann, Radiochimica Acta 51, 145 (1990).

[Armbruster 1997] P. Armbruster, Proceedings of the Robert A. Welch Foundation $41^{\text {st }}$ Conference on Chemical Research, The Transactinide Elements, Houston, 231 (1997).

[Aumann 1974] D. Aumann, G. Müllen, Nuclear Instrumentation and Methods 115, 75 (1974).

[Bondorf 1974] J. P. Bondorf, M. Sobel, and D. Sperber, Physics Reports 15, 83 (1974).

[Borggreen 1970] J. Borggreen, K. Valli, E. K. Hyde. Physical Review C 2, 1841 (1970).

[Colton 1965] R. Colton, The Chemistry of Rhenium and Technetium (Wiley, London, 1965) as referenced by [Pershina 2000a].

[Düllmann 2001a] Ch. E. Düllman, R. Eichler, A. Türler, Paul Scherrer Institut Condensed Matter Research and Material Sciences Progress Report 2000, Annex IIIA Annual Report, Villigen, (2001).

[Düllmann 2001b] Ch. E. Düllman, R. Eichler, H. W. Gäggler, A. Türler, B. Eichler, D. T. Jost, D. Piguet, Paul Scherrer Institut Condensed Matter Research and Material Sciences Progress Report 2000, Annex IIIA Annual Report, Villigen (2001).

[Eichler 1999] R. Eichler, B. Eichler, H. W. Gäggler, D. T. Jost, R. Dressler, A. Türler, Radiochimica Acta, 87, 151 (1999).

[Eichler 2000a] R. Eichler, W. Brüchle, Ch. Düllmann, R. Dressler, B. Eichler, H.W. Gäggeler, K.E. Gregorich, T. Häfeli, U. Kirbach, D.C. Hoffman, S. Hübener, D.T. Jost, V.M. Lavanchy, C.A. Laue, H. Nitsche, J. Patin, D. Piguet, M. Schädel, D. Strellis, S. Taut, L. Tobler, Y. Tsyganov, A. Türler, A.Vahle, P.A. Wilk, A.B. Yakushev, P. Zimmermann, LBNL Annual Report 1999 (2000). 
[Eichler 2000b]

[Eichler 2000c]

[Eicher 2000d]

[Eicher 2001]

[Essel 1987]

[Evans 1972]

[Firestone 1996]

[Fricke 1998]

[Fukuda 1986]
R. Eichler, W. Brüchle, Ch. Düllmann, R. Dressler, B. Eichler, H.W. Gäggeler, K.E. Gregorich, T. Häfeli, U. Kirbach, D.C. Hoffman, S. Hübener, D.T. Jost, V.M. Lavanchy, C.A. Laue, H. Nitsche, J. Patin, D. Piguet, M. Schädel, D. Strellis, S. Taut, L. Tobler, Y. Tsyganov, A. Türler, A.Vahle, P.A. Wilk, A.B. Yakushev, P. Zimmermann, Paul Scherrer Institut Condensed Matter Research and Material Sciences Progress Report 1999, Annex IIIA Annual Report, Villigen, (2000).

R. Eichler, W. Brüchle, Ch. Düllmann, R. Dressler, B. Eichler, H.W. Gäggeler, K.E. Gregorich, T. Häfeli, U. Kirbach, D.C. Hoffman, S. Hübener, D.T. Jost, V.M. Lavanchy, C.A. Laue, H. Nitsche, J. Patin, D. Piguet, M. Schädel, D. Strellis, S. Taut, L. Tobler, Y. Tsyganov, A. Türler, A.Vahle, P.A. Wilk, A.B. Yakushev, P. Zimmermann, Nature 407, 63 (2000).

R. Eichler, B. Eichler, H. W. Gäggleler, D. T. Jost, D. Piguet, A. Türler, Radiochimica Acta 88, 87 (2000).

R. Eichler, C. Düllmann, B. Eichler, H. W. Gäggeler, T. Haefeli, D. T. Jost, D. Piguet, A. Türler, P. Zimmermann, K. E. Gregorich, V. Ninov, C. M. Folden III, U. W. Kirbach, C. A. Laue, D. M. Lee, J. B. Patin, R. Sudowe, P. A. Wilk, H. Nitsche, D. C. Hoffman, Y. Tsyganov, A. B. Yakushev, S. Hübener, S. Taut, A. Vahle, R. Dressler, W. Brüchle, and M. Schädel, LBNL 2000 Annual Report (2001).

H. G. Essel, H. Grein, T. Kroll, W. Kynast, M. Richter, H. Sohlbach, W. Spreng, K. Winkelmann, W. F. J. Müller, IEEE Transactions on Nuclear Science, NS-34, 907 (1987).

J. E. Evans, R. W. Lougheed, M.S. Coops, R.W. Hoff, E. K. Hulet, Nuclear Instrumentation and Methods, 102, 389 (1972).

R. B. Firestone, Table of Isotopes, 8th ed. (Wiley-Interscience, New York, 1996).

B. Fricke, V. Pershina, P. Schwerdtfeger, Gesellschaft für Schwerionenforschung unnumbered publication, Darmstadt, Germany (November 1998).

M. Fukuda et al., RIKEN Accelerator Progress Report 20, 14 (1986). 
[Gäggeler 1994]

[Ghiorso 1970]

[Ghiorso 1971]

[Gregorich 1988]

[Gregorich 1991]

[Gregorich 1992]

[Gregorich 1994]

[Hatsukawa 1990]

[Higgins 1960]

[Hoffman 1990]

[Hoffman 1994]

[Hoffman 2000]
H. W. Gäggeler, et al., Paul Scherrer Institut Condensed Matter Research and Material Sciences Progress Report 1993, Annex IIIA Annual Report, Villigen, Switzerland, 92 (1994).

A. Ghiorso, M. Nurmia, K. Eskola, P. Eskola, Phys. Lett. 32B, 95 (1970).

A. Ghiorso, M. Nurmia, K. Eskola, P. Eskola, Physical Review C 4, 1850 (1971).

K. E. Gregorich, R. A. Henderson, D. M. Lee, M. J. Nurmia, R. M. Chasteler, H. L. Hall, D. A. Bennett, C. M. Gannett, R. B. Chadwick, J. D. Leyba, D. C. Hoffman, Radiochimica Acta, 43, 223 (1988).

K. E. Gregorich, Nuclear Instrumentation and Methods, A 302, 135 (1991).

K. E. Gregorich, H. L. Hall, R. A. Henderson, J. D. Lebya, K. R. Czerwinski, S. A. Kreek, B. A. Kadkhodayan, M. J. Nurmia, D. M. Lee, D. C. Hoffman, Physical Review C 45, 1058-1063 (1992).

K. E. Gregorich, M. R. Lane, M. F. Mohar, D. M. Lee, C. D. Kacher, E. R. Sylwester, and D. C. Hoffman. Physical Review Letters 72, 1423 (1994).

Y. Hatsukawa, H. Nakahara, D. C. Hoffman, Physical Review C 42, 674 (1990).

G. H. Higgins, The Radiochemistry of the Transcurium Elements, NAS-31 3031 (National Academy of Sciences National Research Council, Washington D.C.,1960).

D. C. Hoffman, D. M. Lee, K. E. Gregorich, M. J. Nurmia, R. B. Chadwick, K. B. Chen, K. R. Czerwinski, C. M. Gannett, H. L. Hall, R. A. Henderson, B. Kadkhodayan, S. A. Kreek, and J. D. Leyba, Physical Review C 41, 631 (1990).

D. C. Hoffman, Chemical and Engineering News 72, 24 (1994).

D. C. Hoffman, A. Ghiorso, G. T. Seaborg, The Transuranium People: The Inside Story (Imperial College Press, London, 2000). 
[Hofmann 1995]

[Hyde 1987]

[IUPAC 1997]

[Johansson 2001]

[Johnson 1999]

[Kirbach 2000]

[Kirbach 2001]

[Kirby 1959]

[Kirby 1986]

[Kratz 1989]

[Kratz 1992]
S. Hofmann, V. Ninov, F. P. Heßberger, P. Armbruster, H. Folger, G. Münzenberg, H. J. Schött, A. G. Popeko, A. V. Yeremin, A. N. Andreyev, S. Saro, R. Janik, M. Leino, Zeitschrift für Physic A 350, 281 (1995).

E. K. Hyde, D. C. Hoffman, O. L. Keller, Radiochemica Acta 42, 57 (1987).

International Union of Pure and Applied Chemistry, Inorganic Chemistry Division, Commission on Nomenclature of Inorganic Chemistry, Pure and Applied Chemistry 69, 2471 (1997).

M. Johansson, Ph.D. Dissertation (University of Oslo, Norway, 2001).

E. Johnson, V. Pershina, B. Fricke, Journal of Physical Chemistry A 103, 8458 (1999).

U.W. Kirbach, K.E. Gregorich, V. Ninov, D.M. Lee, J.B. Patin, D.A. Shaugnessy, D.A. Strellis, P.A. Wilk, D.C. Hoffman, and H. Nitsche, "The Recoil product Transfer Chamber (RTC)", LBNL Annual Report 1999 (2000).

U. W. Kirbach, private communication (2001).

H. W. Kirby, The Radiochemistry of Protactinium, NAS-NS 3016, (National Academy of Sciences National Research Council, Washington D.C., 1959).

H. W. Kirby, "Chapter 4: Protactinium" in The Chemistry of the Actinide Elements, $2^{\text {nd }}$ edition, edited by J. J. Katz, G. T. Seaborg, L. R. Morss (Chapman and Hall, New York, 1986).

J. V. Kratz, H. P. Zimmermann, U. W. Scherer, M. Schädel, W. Brüchle, K. E. Gregorich, C. M. Gannett, H. L. Hall, R. A. Henderson, D. M. Lee, J. D. Leyba, M. J. Nurmia, D. C. Hoffman, H. Gäggeler, D. Jost, U. Baltensperger, Ya Nai-Qi, A. Türler, Ch. Lienert, Radiochimica Acta 48, 121 (1989).

J. V. Kratz, M. K. Gober, H. P. Zimmermann, M. Schädel, W. Brüchle, E. Schimpf, K. E. Gregorich, A. Türler, N. J. Hannink, K. R. Czerwinski, B. Kadkhodayan, D. M. Lee, M. J. Nurmia, D. C. Hoffman, H. Gäggeler, D. Jost, J. Kovacs, U. W. Scherer, A. Weber, Physical Review C 45, 1064 (1992). 
[Lane 1996]

[Lazarev 1994]

[Lee 1982]

[Liang 1996]

[Lougheed 1997a]

[Lougheed 1997b]

[Malmbeck 2000]

[McFarland 1982]

[Möller 1998]

[Moody 1983]

[Morozov 1971]

[Müllen 1975]

[Münzenberg 1981]
M. R. Lane, K. E. Gregorich, D. M. Lee, M. F. Mohar, M. Hsu, C. D. Kacher, B. Kadkhodayan, M. P. Neu, N. J. Stoyer, E. R. Sylwester, J. C. Yang, and D. C. Hoffman, Physical Review C 53, 2393 (1996)

Yu. A. Lazarev, Yu. V. Lobanov, Yu. Ts. Oganessian, V. K. Utyonkov, F. Sh. Abdullin, G. V. Buklanov, B. N. Gikal, S. Iliev, A. N. Mezentsev, A. N. Polyakov, I. M. Sedykh, I. V.

Shirokovsky, V. G. Subbotin, A. M. Sukhov, Yu. S. Tsyganov, V. E. Zhuchko, Physical Review Letters 73, 624 (1994).

D. Lee, H. von Gunten, B. Jacak, M. Nurmia, Y. F. Liu, C. Luo, G. Seaborg, and D. C. Hoffman, Physical Review C 25, 286 (1982).

C. F. Liang, P. Paris, A. Plochocki, E. Ruchowska, A. Gizon, D. Barnéoud, J. Genevey, G. Cãta, and R. K. Sheline, Zeitschrift für Physik A 354, 153 (1996).

R. Lougheed, Proceedings of the Robert A. Welch Foundation $41^{\text {st }}$ Conference on Chemical Research, The Transactinide Elements, Houston, 307 (1997).

R. Lougheed, Nature 388, 21 (1997).

R. Malmbeck, G. Skarnemark, J. Alstad, K. Fure, M. Johansson, J. P. Omtvedt, Journal of Radioanalytical and Nuclear Chemistry 246, 349 (2000).

R. McFarland, Lawrence Berkeley National Laboratory Report LBL-15027 (1982).

P. Möller, mass calculations at http://t2.lanl.gov/data/astro/molnix/massd.html

K. J. Moody, Lawrence Berkeley National Laboratory Report, LBL-16249 (1983).

A. I. Morozov, E. V. Karlova, Russian Journal of Inorganic Chemistry 16, 12 (1971).

G. Müllen, D. C Aumann, Nucl. Instrum. Methods 128, 425 (1975).

G. Münzenberg, S. Hofmann, F. P. Heßberger, W. Reisdorf, K. H. Schmidt, J. H. R. Schneider, P. Armbruster, Z. Phys. A 300, 107 (1981). 
[Münzenberg 1989] G. Münzenberg, P. Armbruster, S. Hofmann, F. P. Heßberger, H. Folger, J. G. Keller, V. Ninov, K. Poppensieker, A. B. Quint, W. Reisdorf, K. H. Schmidt, J. H. R. Schneider, H. J. Schött, K.

Sümmerer, I. Zychor, M. E. Leino, D. Ackermann, U. Gollerthan, E. Hanelt, W. Morawek, D. Vermeulen, Y. Fujita, T. Schwab, Z. Phys. A 333, 163 (1989).

[Nash 1995] C. S. Nash, B. E. Bursten, New Journal of Chemistry 19, 669 (1995).

[Oganessian 1976a] Yu. Ts. Oganesyan, A. G. Demin, N. A. Danilov, M. P. Ivanov, A. S. Il'nov, N. N. Kolesnikov, B. N. Markov, V. M. Plotko, S. P. Tret'yakova, G. N. Flerov, JETP Letters 23, 277 (1976).

[Oganessian 1976b] Yu. Ts. Oganesyan, A. G. Demin, N. A. Danilov, G. N. Flerov, M. P. Ivanov, A. S. Iljinov, N. N. Kolesnikov, B. N. Markov, V. M. Plotko, S. P. Tretyakova, Nuclear Physics A273, 505 (1976).

[Oganessian 1983] Yu. Ts. Oganesyan, JINR-D-7-83-433, also as International School-Seminar on Heavy Ion Physics (1983), in Russian.

[Oganessian 1997] Yu. Ts. Oganessian, Proceedings of the Robert A. Welch Foundation $41^{\text {st }}$ Conference on Chemical Research, The Transactinide Elements, Houston, 347 (1997).

[Omtvedt 1998] J. P. Omtvedt, J. Alstad, K. Eberhardt, K. Fure, R. Malmbeck, M. Mendel, A. Nähler, G. Skarnemark, N. Trautmann, N. Wiehl, B. Wierczinski, Journal of Alloys and Compounds 271-273, 303, (1998).

[Omtvedt 2001] J. P. Omtvedt, Private Communication (2001).

[Paulus 1998] W. Paulus, J. V. Kratz, E. Strub, S. Zauner, W. Brüchle, V.

Pershina, M. Schädel, B. Schausten, J. L. Adams, K. E. Gregorich, D. C. Hoffman, M. R. Lane, C. Laue, D. M. Lee, C. A. McGrath, D. K. Shaughnessy, D. A. Strellis, E. R. Sylwester, Journal of Alloys and Compounds 271-273, 292 (1998).

[Paulus 1999] W. Paulus, J. V. Kratz, E. Strub, S. Zauner, W. Brüchle, V. Pershina, M. Schädel, B. Schausten, J. L. Adams, K. E. Gregorich, D. C. Hoffman, M. R. Lane, C. Laue, D. M. Lee, C. A. McGrath, D. K. Shaughnessy, D. A. Strellis, E. R. Sylwester, Radiochimica Acta 84, 69 (1999).

[Pershina 1992a] V. Pershina, W. Đ. Sepp, B. Fricke, A. Rosén, Journal of Chemical Physics, 96, 8367 (1992). 
[Pershina 1992b] V. Pershina, W. Đ. Sepp, B. Fricke, D. Kolb, M. Schädel, G. V. Ionova, Journal of Chemical Physics, 97, 1116 (1992).

[Pershina 1992c] V. Pershina, W. Đ. Sepp, T. Bastug, B. Fricke, G. V. Ionova, Journal of Chemical Physics, 97, 1123 (1992).

[Pershina 1993] V. Pershina, B. Fricke, Journal of Chemical Physics, 99, 9720 (1993).

[Pershina 1994] V. Pershina, B. Fricke, J. V. Kratz, G. V. Ionova, Radiochimica Acta 64, 37 (1994).

[Pershina 1995] V. Pershina, B. Fricke, Journal of Physical Chemistry 99, 144 (1995).

[Pershina 1996a] V. Pershina, Chemical Reviews, 96, 1977 (1996).

[Pershina 1996b] V. Pershina, B. Fricke, Journal of Physical Chemistry 100, 8748 (1996) \& Erratum, Journal of Physical Chemistry 100, 17434 (1996)

[Pershina 1998a] V. Pershina, Radiochimica Acta 80, 65 (1998).

[Pershina 1998b] V. Pershina, Radiochimica Acta 80, 75 (1998).

[Pershina 1999a] V. Pershina, E. Johnson, B. Fricke, Journal of Physical Chemistry A, 103, 8463 (1999).

[Pershina 1999b] V. Pershina, T. Bastug, Radiochimica Acta 84, 79 (1999).

[Pershina 2000a] V. Pershina, T. Bastug, Journal of Chemical Physics 113, 1441 (2000).

[Pershina 2000b] V. Pershina, J. V. Kratz, Inorganic Chemistry, Published electronically on the web, ACS.Org (2000).

[Pfrepper 2000] G. Pfrepper, R. Pfrepper, A. Kronenberg, J. V. Kratz, A. Nähler, W. Brüchle, M. Schädel, Radiochimica Acta 88, 273 (2000).

[Pyykkö 1988] P. Pyykkö, Chem. Rev. 88, 563 (1988).

[Rathbun 1991] W. Rathbun, Lawrence Berkeley National Laboratory Report No. LBL-29734 (1991).

[Reisdorf 1992] W. Reisdorf, M. Schädel, Zeitshrift für Physik A 343, 47 (1992).

[Schädel 1995a] M. Schädel, Radiochimica Acta 70/71, 207 (1995). 
[Schädel 1995b]

[Schädel 1997]

[Schädel 1998]

[Schumann 1998]

[Seaborg 1945]

[Seth 1995]

[Smolańczuk 1995]

[Smolańczuk 1997]

[Steinberg 1961]

[Strub 2001]

[Sudowe 2001]

[Swiatecki 1996]

[Sylwester 1998]
M. Schädel, E. Jäger, W. Brüchle, K. Sümmerer, E. K. Hulet, J. F. Wild, R. W. Jougheed, R. J. Dougan, K. J. Moody, Radiochemical Acta 68, 7 (1995).

M. Schädel, W. Brüchle, R. Dressler, B. Eichler, H. W. Gäggeler, R. Günther, K. E. Gregorich, D. C. Hoffman, S. Hübener, D. T. Jost, J. V. Kratz, W. Paulus, D. Schumann, S. Timokhin, N. Trautmann, A. Türler, G. Wirth, A. Yakuschev, Nature 388, 55 (1997).

M. Schädel, Journal of Alloys and Compounds 271-273, 312 (1998).

D. Schumann, H. Nitsche, St. Taut, D. T. Jost, H. W. Gäggler, A. B. Yakushev, G. V. Buklanov, V. P. Domanov, Din Thi Lien, B. Kubica, R. Misiak, Z. Szeglowski, Journal of Alloys and Compounds 271-273, 307 (1998).

G. T. Seaborg, Chemical and Engineering News, 23, 2190 (1945).

M. Seth, M. Dolg, P. Fulde, P. Schwerdtfeger, Journal of the American Chemical Society 117, 6597 (1995).

R. Smolańczuk, J. Skalski, and A. Sobiczewski, Physical Review C 52, 1871 (1995).

R. Smolańczuk, Physical Review C, 56, 812 (1997).

E. P. Steinberg, The Radiochemistry of Niobium and Tantalum, NAS-NS 3039, (National Academy of Sciences National Research Council, Washington D.C., 1961).

E. Strub, J. V. Kratz, A. Kronenberg, A. Nähler, P. Thörle, W. Brüchle, E. Jäger, Z. Li, M. Schädel, B. Schausten, E. Schimpf, D. Jost, A. Türler, H. W. Gäggeler, J. P. Glatz, Paul Scherrer Institut Condensed Matter Research and Material Sciences Progress Report 2000, Annex IIIA Annual Report, Villigen (2001).

R. Sudowe, K. E. Gregorich, D. M. Lee, H. Nitsche, D. C. Hoffman, LBNL 2000 Annual Report (2001).

W. Swiatecki, Private Communication, text file of Thomas Fermi model masses.

E. R. Sylwester, Ph.D. Thesis, University of California, Berkeley, LBNL-42352 (1998). 
[Takahashi 1973] K. Takahashi, M. Yamada, T. Kondoh. At. Data Nucl. Data Tables 12, 101 (1973).

[Tove 1958] P. A. Tove, Arkiv Fysik. 13, 549 (1958).

[Trautmann 1996] N. Trautmann, Radiochimica Acta 70/71, 237 (1995).

[Türler 1996] A. Türler, B. Eichler, D. T. Jost, D. Piguet, H. W. Gäggeler, K. E. Gregorich, B. Kadkhodayan, S. A. Kreek, D. M. Lee, M. Mohar, E. Sylwester, D. C. Hoffman, S. Hübener, Radiochimica Acta 73, 55 (1996).

[Türler 1998a] A. Türler, R. Dressler, B. Eichler, H. W. Gäggeler, D. T. Jost, M. Schädel, W. Brüchle, K. E. Gregorich, N. Trautmann, S. Taut, Physical Review C 57, 1648 (1998).

[Türler 1998b] A. Türler, G. V. Buklanov, B. Eichler, H. W. Gäggeler, M. Grantz, S. Hübener, D. T. Jost, V. Ya. Lebedev, D. Piguet, S. N. Timokhin, A. B. Yakushev, I. Zvara, Journal of Alloys and Compounds 271273, 287 (1998).

[Türler 1999] A. Türler, Habilitationsshrift (Habilitation Dissertation), (Universität Bern, Switzerland, 1999).

[Varga 2000] S. Varga, B. Fricke, M. Hirata, T. Baştuğ, V. Pershina, S. Fritzsche, Journal of Physical Chemistry A 104 (2000).

[Wild 1988] J. F. Wild, private communication (1988); T. Sikkeland, Ark. Fys. 36, 539 (1967); J. Alonso, edited by R. Warncke, in Gmelin Hanbuch der Anorganishen Chemie, Vol. 7b, p. 28, (SpringerVerlag, New York, 1974).

[Wierczinski 1994] B. Wierczinski, Doctoral Dissertation (Universität Mainz, Germany, 1994), in german.

[Wierczinski 1995] B. Wierczinski, J. Alstad, K. Eberhardt, B. Eichler, H. Gäggler, G. Herrmann, D. Jost, A. Nähler, M. Pense-Maskow, A.V. V. Reddy, G. Skarnemark, N. Trautmann, A. Türler, Radiochimica Acta 69, 77 (1995).

[Wierczinski 1996a] B. Wierczinski, D. C. Hoffman, Instrumentation for Atom-at-a Time Chemistry of the Heavy Elements (LBNL-37463, 1995), edited by Sood, Reddy, Pujari, in Frontiers in Nuclear Chemistry, (Perfect Prints, Thane, India, 1996). 
[Wierczinski 1996b] B. Wierczinski, K. Eberhardt, G. Herrmann, J. V. Kratz, M. Mendel, A. Nähler, F. Rocker, U. Tharun, N. Trautmann, K. Weiner, N. Wiehl, J. Alstad, G. Skarnemark, Nuclear Instrumentation and Methods A 370, 532 (1996).

[Wierczinski 2001] B. Wierczinski, K. E. Gregorich, B. Kadkhodayan, D. M. Lee, L. G. Beauvais, M. B. Hendricks, C. D. Kacher, M. R. Lane, D. A. Keeney-Shaughnessey, N. J. Stoyer, D. A. Strellis, E. R. Sylwester, P. A. Wilk, D. C. Hoffman, R. Malmbeck, G. Skarnemark, J. Alstad, J. P. Omtvedt, K. Eberhardt, M. Mendel, A. Nähler, N. Trautmann, Radioanalytical and Nuclear Chemistry, accepted for publication (2001).

[Wilk 1995] Philip Wilk, "Time Resolved Resonance Raman Spectroscopy of Chromium Pentacarbonyl”, Reed College Thesis (1995).

[Wilk 1996]

Philip A. Wilk and Darleane C. Hoffman, "Off-line Generation of ${ }^{233} \mathrm{~Pa}$ and the ${ }^{224} \mathrm{~Pa}$ Yield Through SISAK", LBNL Annual Report (1996).

[Wilk 1997] P. A. Wilk, K. E. Gregorich, M. B. Hendricks, M. R. Lane, D. M. Lee, C. A. McGrath, D. A. Shaughnessy, D. A. Strellis, E. R. Sylwester, D. C. Hoffman, Physical Review C 56, 1626-8 (1997) \& Erratum, Phys. Rev. C 58, 1352 (1998).

[Wilk 2000] P. A. Wilk, K. E. Gregorich, A. Türler, C. A. Laue, R. Eichler, V. Ninov, J. L. Adams, U. W. Kirbach, M. R. Lane, D. M. Lee, J. B. Patin, D. A. Shaughnessy, D. A. Strellis, H. Nitsche, and D. C. Hoffman, Physical Review Letters 85, 2697 (2000).

[Zhuikov 1990] B. L. Zhuikov, V. A. Glebov, V. S. Nefedov, I. Zvara, Journal of Radioanalytical and Nuclear Chemistry 143, 103 (1990).

[Zvára 1971] I. Zvára, V. Z. Belov, L. P. Chelnokov, V. P. Domanov, M. Hussonois, Yu. S. Korotkin, V. A. Schegolev, M. R. Shalayevsky, Inorganic Nuclear Chemistry Letters 7, 1109 (1971).

[Zvára 1976] I. Zvara, V. Z. Belov, V. P. Domanov, M. R. Shalaevskii, Soviet Radiochemistry 18, 328 (1976); translated from Radiokhimiya 18, 371 (1984), in russian.

[Zvára 1982] I. Zvara, Radiochimica Acta 38, 95 (1982). 
[Zvára 1984] I. Zvara, V. P. Domanov, Z. Khyubener, M. R. Shalavskii, S. N. Timokhin, B. L. Zhuikov, B. Aikhler, G. V. Buklanov. Soviet Radiochemistry 26, 72 (1984); translated from Radiokhimiya 26, 76 (1984), in russian. 